\title{
Arene-Perfluoroarene Interactions in Solution
}

\author{
Ga Young Lee ${ }^{1}$, Elizabeth $\mathrm{Hu}^{1}$, Arnold L. Rheingold ${ }^{2}$, K. N. Houk ${ }^{1 *}$, and Ellen, M. Sletten ${ }^{*}$ \\ ${ }^{1}$ Department of Chemistry and Biochemistry, University of California, Los Angeles, California 90095, United States \\ ${ }^{2}$ Department of Chemistry and Biochemistry, University of California, San Diego, California 92093, United States
}

\section{Table of Contents}

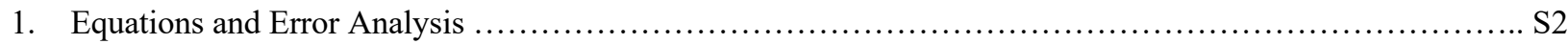

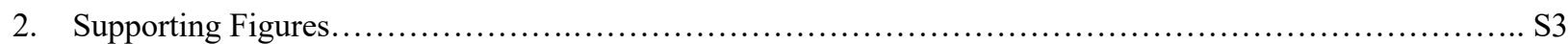

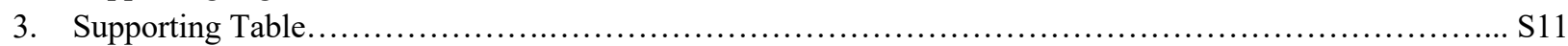

4. ${ }^{1} \mathrm{H}$ NMR titration data

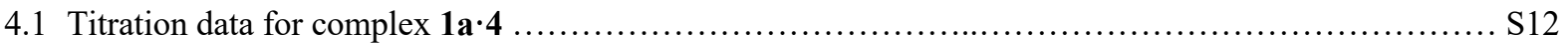

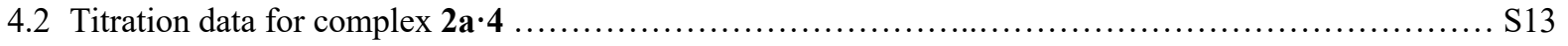

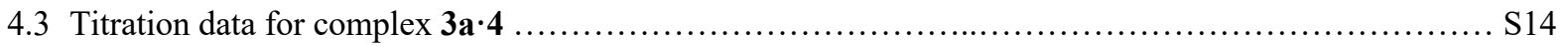

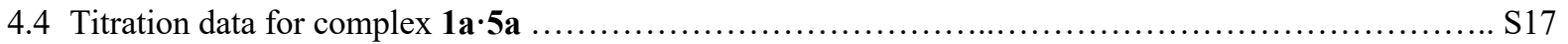

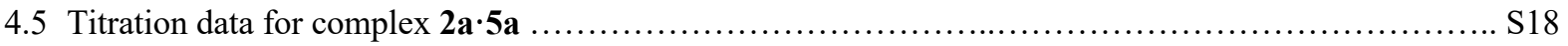

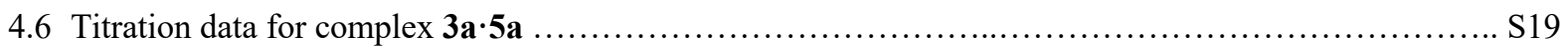

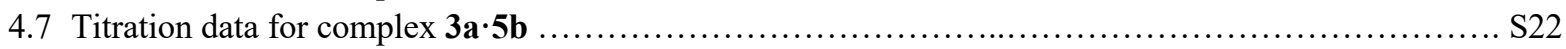

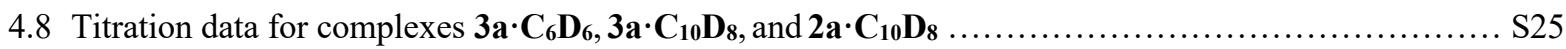

5. Crystallographic Information

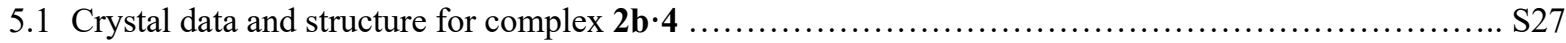

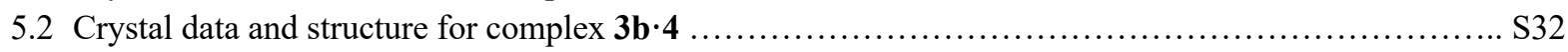

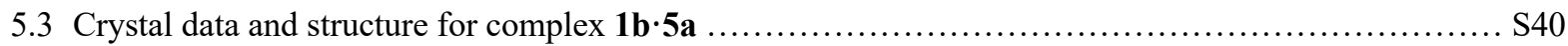

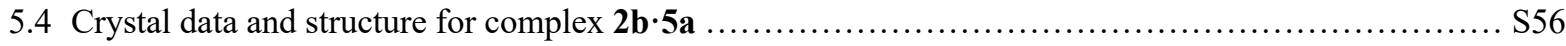

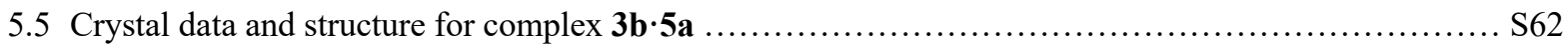

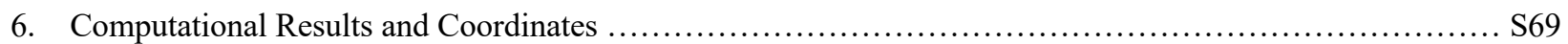

7. NMR Spectra of compounds S1, 1a, 2a, 3a, 3b, S2, S3, 5b, S4, and S5 ................................ S73 


\section{Equations and Error Analysis}

EQN 1. 1:1 H:G binding mode

$\Delta \delta=\delta_{\Delta \mathrm{HG}}\left(\frac{[\mathrm{HG}]}{[\mathrm{H}]_{0}}\right)$ where $[\mathrm{HG}]=\frac{1}{2}\left\{\left([\mathrm{G}]_{0}+[\mathrm{H}]_{0}+\frac{1}{K_{a}}\right)-\sqrt{\left([\mathrm{G}]_{0}+[\mathrm{H}]_{0}+\frac{1}{K_{a}}\right)^{2}-4[\mathrm{H}]_{0}[\mathrm{G}]_{0}}\right\}$

EQN 2. 1:2 H:G binding mode

$$
\Delta \delta=\frac{\delta_{\Delta \mathrm{HG}}[\mathrm{H}]_{0} K_{1}[\mathrm{G}]+\delta_{\Delta \mathrm{HG}_{2}}[\mathrm{H}]_{0} K_{1} K_{2}[\mathrm{G}]^{2}}{1+K_{1}[\mathrm{G}]+K_{1} K_{2}[\mathrm{G}]^{2}} \text { where } K_{1}=\frac{[\mathrm{HG}]}{[\mathrm{H}][\mathrm{G}]} \text { and } K_{2}=\frac{\left[\mathrm{HG}_{2}\right]}{[\mathrm{H}][\mathrm{HG}]}
$$

EQN 3. 2:1 H:G binding mode

$$
\Delta \delta=\frac{\delta_{\Delta \mathrm{HG}}[\mathrm{G}]_{0} K_{1}[\mathrm{H}]+2 \delta_{\Delta \mathrm{H}_{2} \mathrm{G}}[\mathrm{G}]_{0} K_{1} K_{2}[\mathrm{H}]^{2}}{1+K_{1}[\mathrm{H}]+K_{1} K_{2}[\mathrm{H}]^{2}} \text { where } K_{1}=\frac{[\mathrm{HG}]}{[\mathrm{H}][\mathrm{G}]} \text { and } K_{2}=\frac{\left[\mathrm{H}_{2} \mathrm{G}\right]}{[\mathrm{H}][\mathrm{HG}}
$$

EQN 4. $K_{a}$ value determination for 1 titration experiment

$$
K_{a 1, a v g}=\left(\frac{K_{a 1, a}+K_{a 1, b}+K_{a 1, c}}{3}\right)
$$

where $K_{a, a}, K_{a, b}, K_{a, c}$ are $K_{a}{ }^{\prime}$ s determined from proton a, b, and c, respectively

EQN 5. Average $K_{a}$ value determination from duplicate experiments

$$
K_{a, a v g}=\left(\frac{K_{a 1, a v g}+K_{a 2, a v g}}{3}\right)
$$

where $K_{a 1, a v g}$ and $K_{a 2, a v g}$ are average $K_{a}^{\prime}$ s determined from first and second titration experiments

EQN 6. $K_{a}$ error determination

$$
\text { error }=0.95 * \frac{\left[\frac{\left(K_{a 1, a v g}-K_{a, a v g}\right)^{2}+\left(K_{a 2, a v g}-K_{a, a v g}\right)^{2}}{2}\right]}{\sqrt{2}}
$$




\section{Supporting Figures}

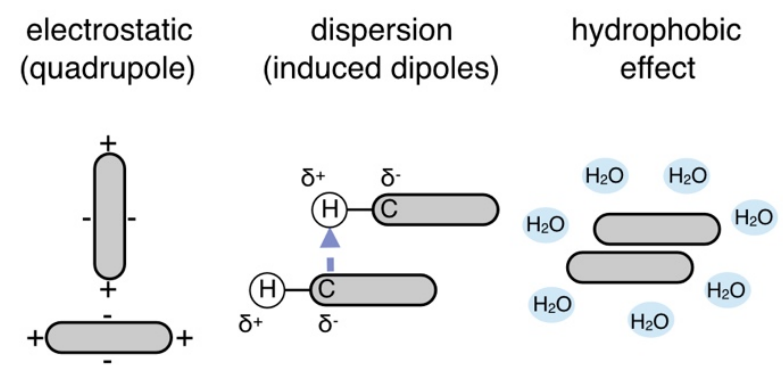

Figure S1. Three main stabilizing factors for arene-arene interactions in solution.

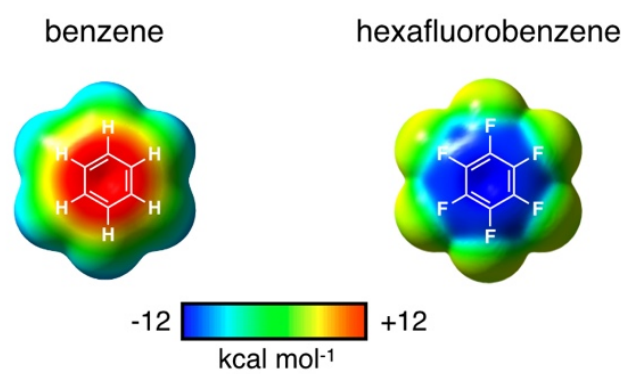

Figure S2. (a) Electrostatic potential maps (ESP) of benzene (left) and hexafluorobenzene (right), computed with $\mathrm{B} 3 \mathrm{LYP} / 6-31 \mathrm{G}(\mathrm{d})$ and plotted with a $\pm 12 \mathrm{kcal} \mathrm{mol}^{-1}$ range. Benzene and hexalfuorobenzene have opposite quadrapoles. 
(a)

Synthesis of TEGylated arenes

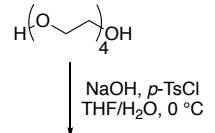

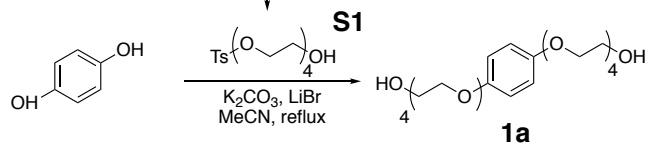
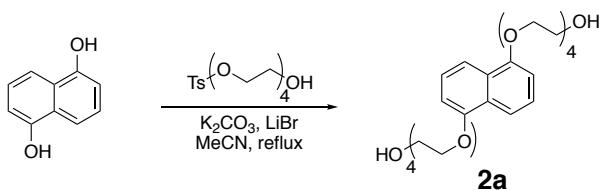

(c)

Synthesis of fluoroarenes $\left(\mathrm{H}_{2} \mathrm{O}\right.$-soluble $)$
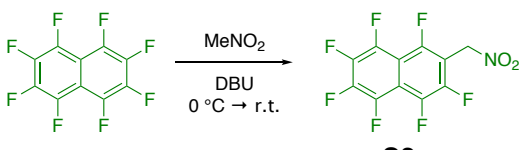

S2

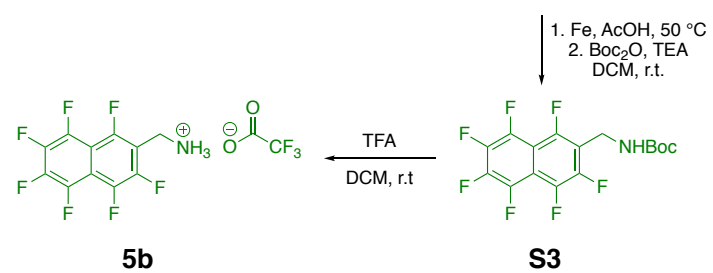

(d)

Synthesis of fluoroarenes ( $\mathrm{H}_{2} \mathrm{O}$-insoluble)

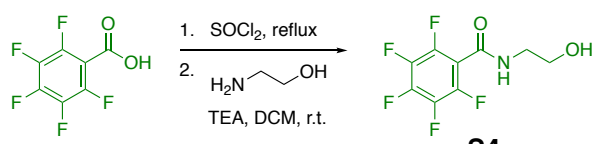

(b) Synthesis of methylated arene

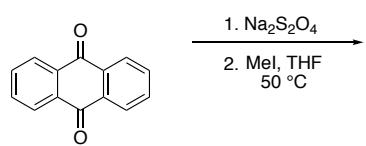

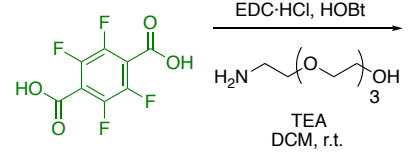

TCM, r.t.
S4<smiles>O=C(NCCOCCO)C(=O)NCCOCCOCCOCCOCCOCCO</smiles>

S5

Figure S3. General synthetic scheme of electron-rich arenes (black) and electron-deficient fluoroarenes (green). (a) Synthesis of TEGylated arenes for binding affinity assays. (b) Synthesis of methylated arene for crystallography. (c) Synthesis of water soluble fluoroarenes for binding affinity assays. (d) Synthesis of fluoroarenes that were not soluble in water. 

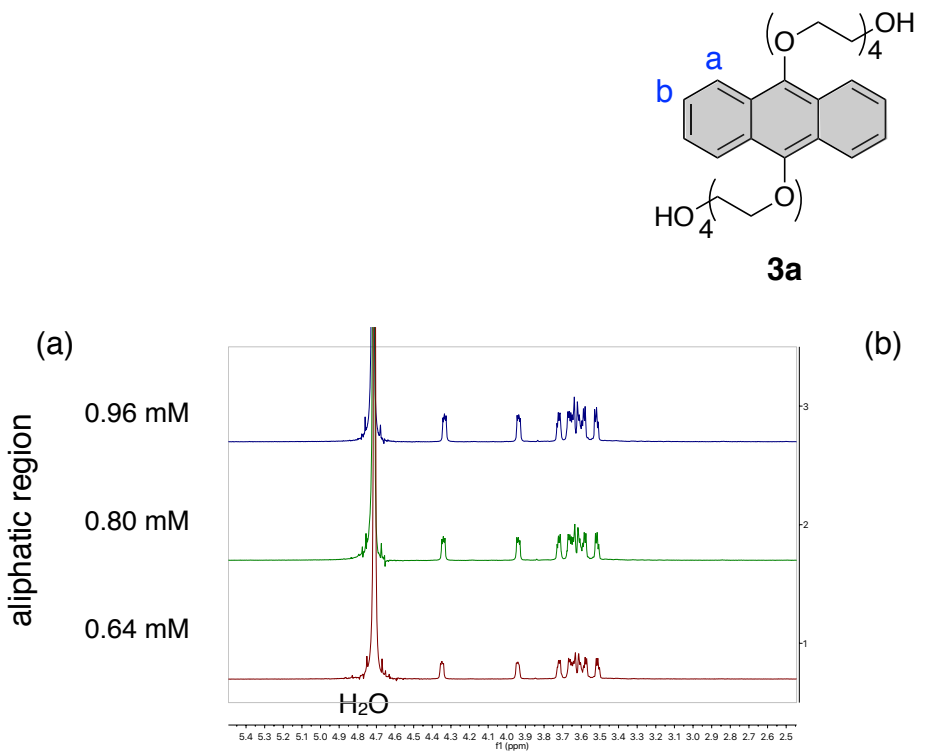

(b)
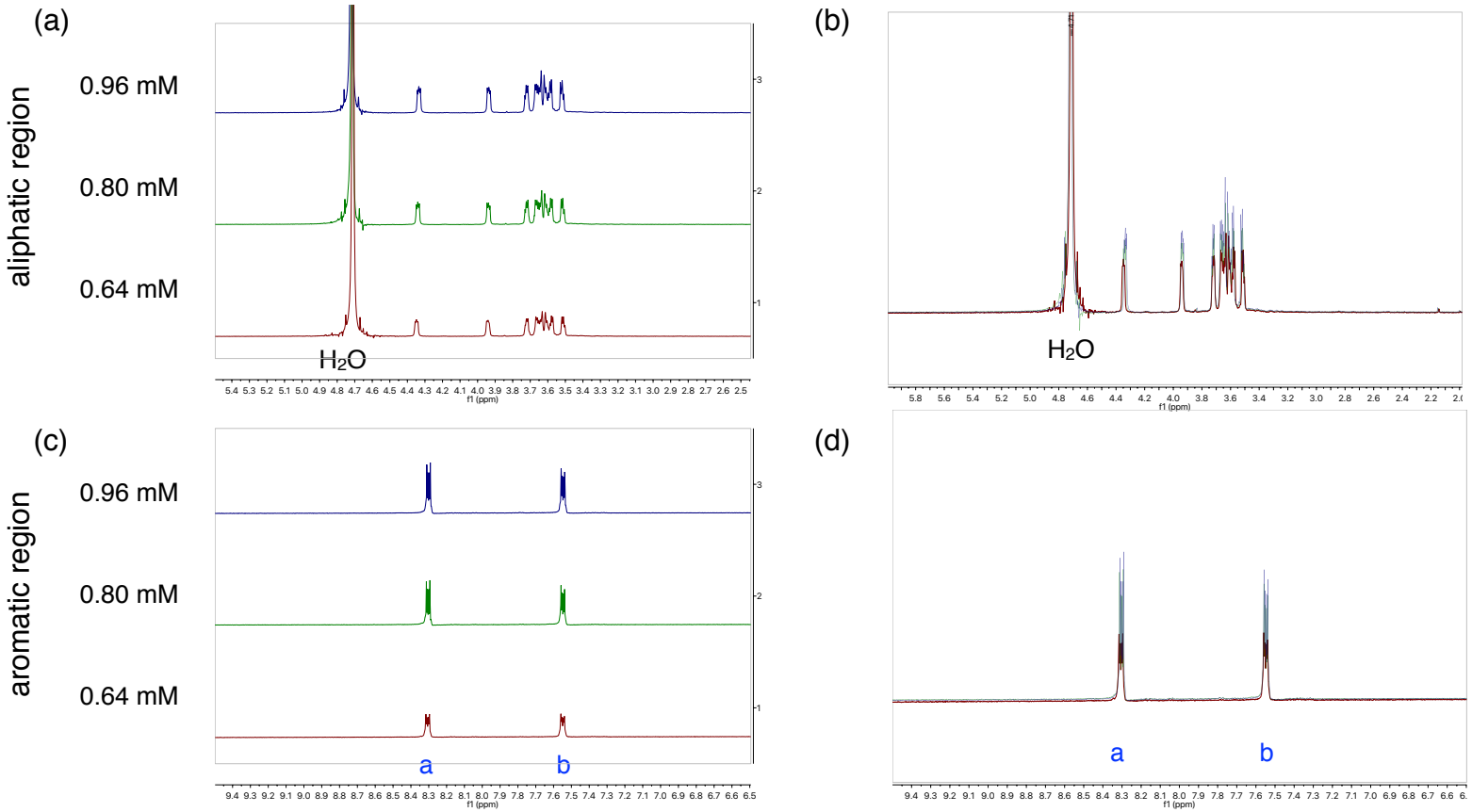

(d)

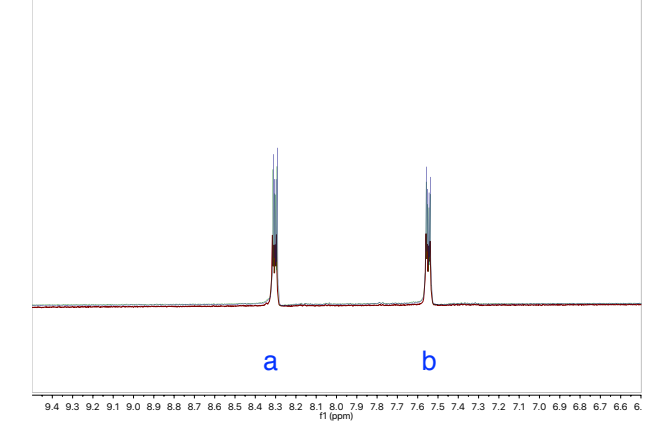

Figure S4. Concentration dependent ${ }^{1}$ H NMR spectra of arene host 3a. Original $[\mathrm{H}]_{0}$ employed for titration studies is $0.8 \mathrm{mM}$, and therefore, we tested $0.64 \mathrm{mM}$ (lower) and $0.96 \mathrm{mM}$ (higher) concentrations. The NMR spectra show no significant changes, suggesting that anthracene host 3a is not self-aggregated at the concentration employed for titration study (0.8mM). Stacked (a) and overlayed (b) NMR spectra in the aliphtatic region. Stacked (c) and overlayed (d) NMR spectra in the aromatic region. 

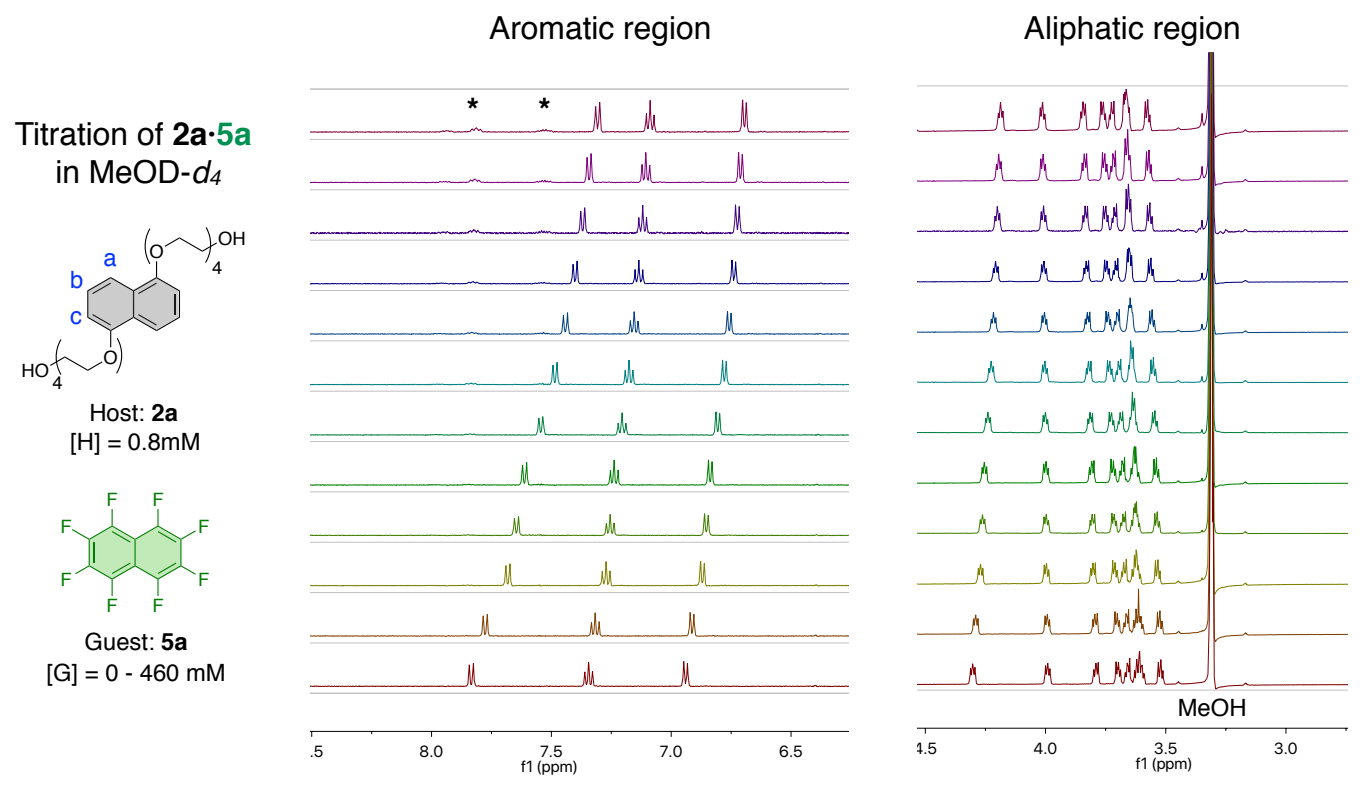

Figure S5. ${ }^{1} \mathrm{H}$ NMR titration in the aromatic and aliphatic region of complex 2a.5a in MeOD- $d_{4}$. Chemical shifts shown are aromatic region (left) and aliphatic region (right) of arene 2a. Major shifts occur on the aromatic region where the shifts in the aliphatic region are relatively small. * = octafluoronapthalene impurities. 
${ }^{1} \mathrm{H}$ NMR $\left(376 \mathrm{MHz}\right.$, Acetonitrile- $\left.d_{3}\right)$

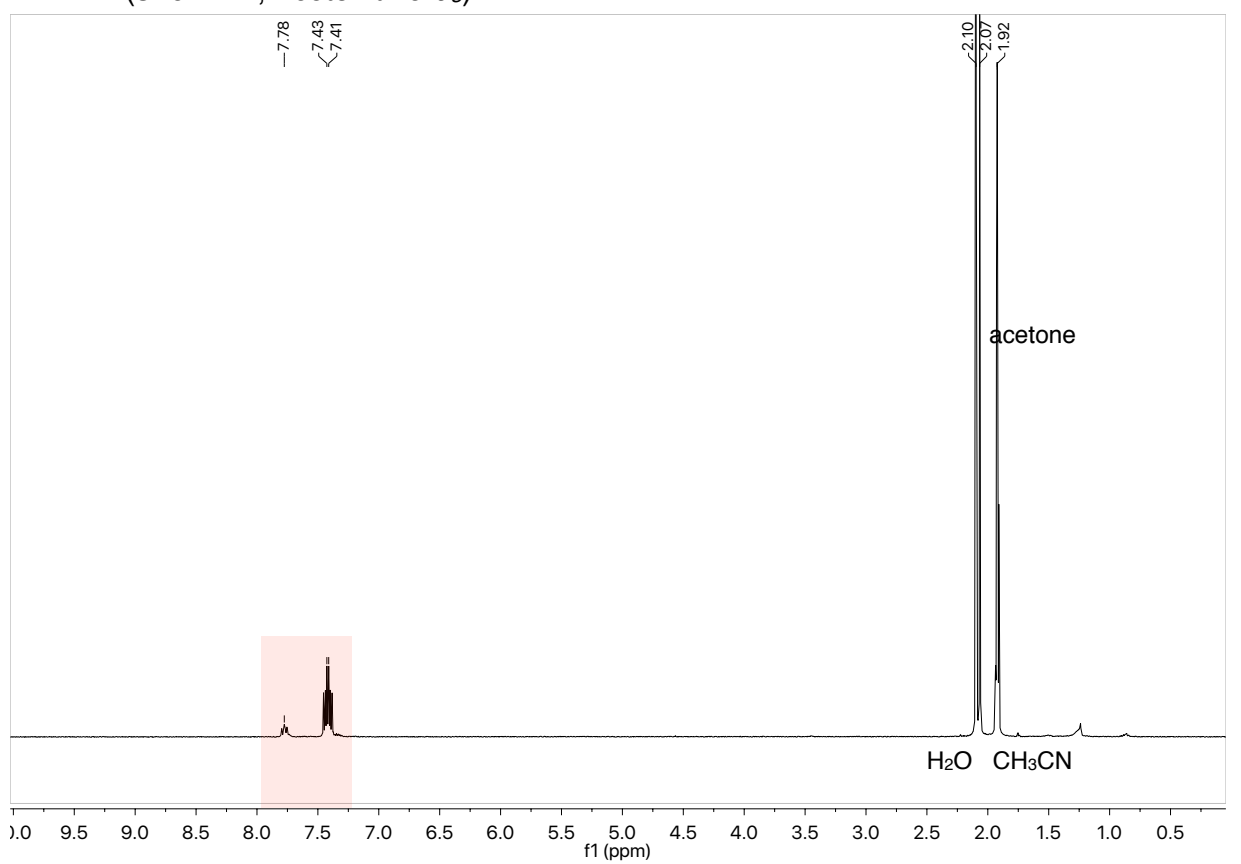

19F NMR (376 MHz, Acetonitrile- $\left.d_{3}\right)$

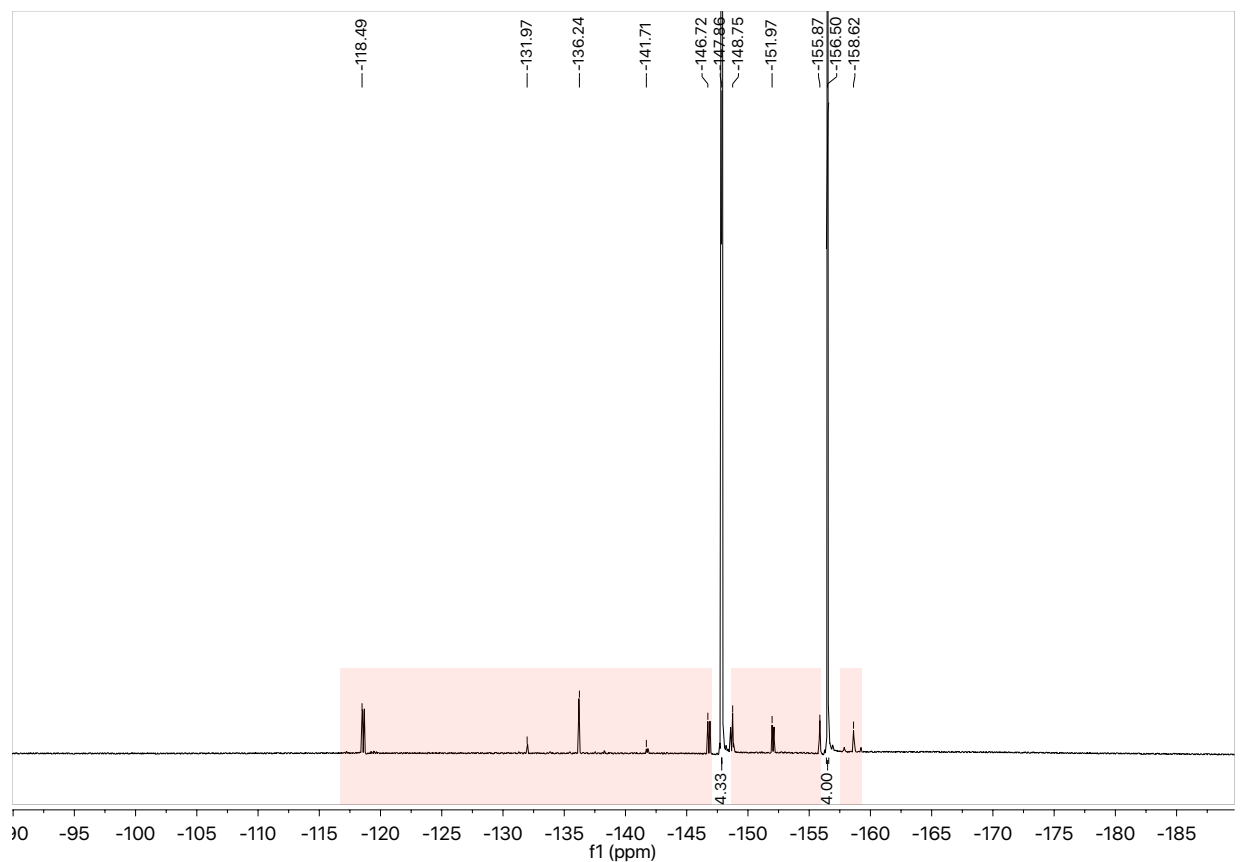

Figure S6. ${ }^{1} \mathrm{H}$ and ${ }^{19}$ F NMR spectra of commercially available octafluoronaphthalene, 5a. Red highlighted regions indicate the impurities, which were found to be at least 2 isomers of partially hydrogenated

perfluoronapthalene. HR-GC/MS (EI): Calculated for $\mathrm{C}_{10} \mathrm{HF}_{7}[\mathrm{M}]+$ : 253.9967; Found 253.9955 and 253.9957. 


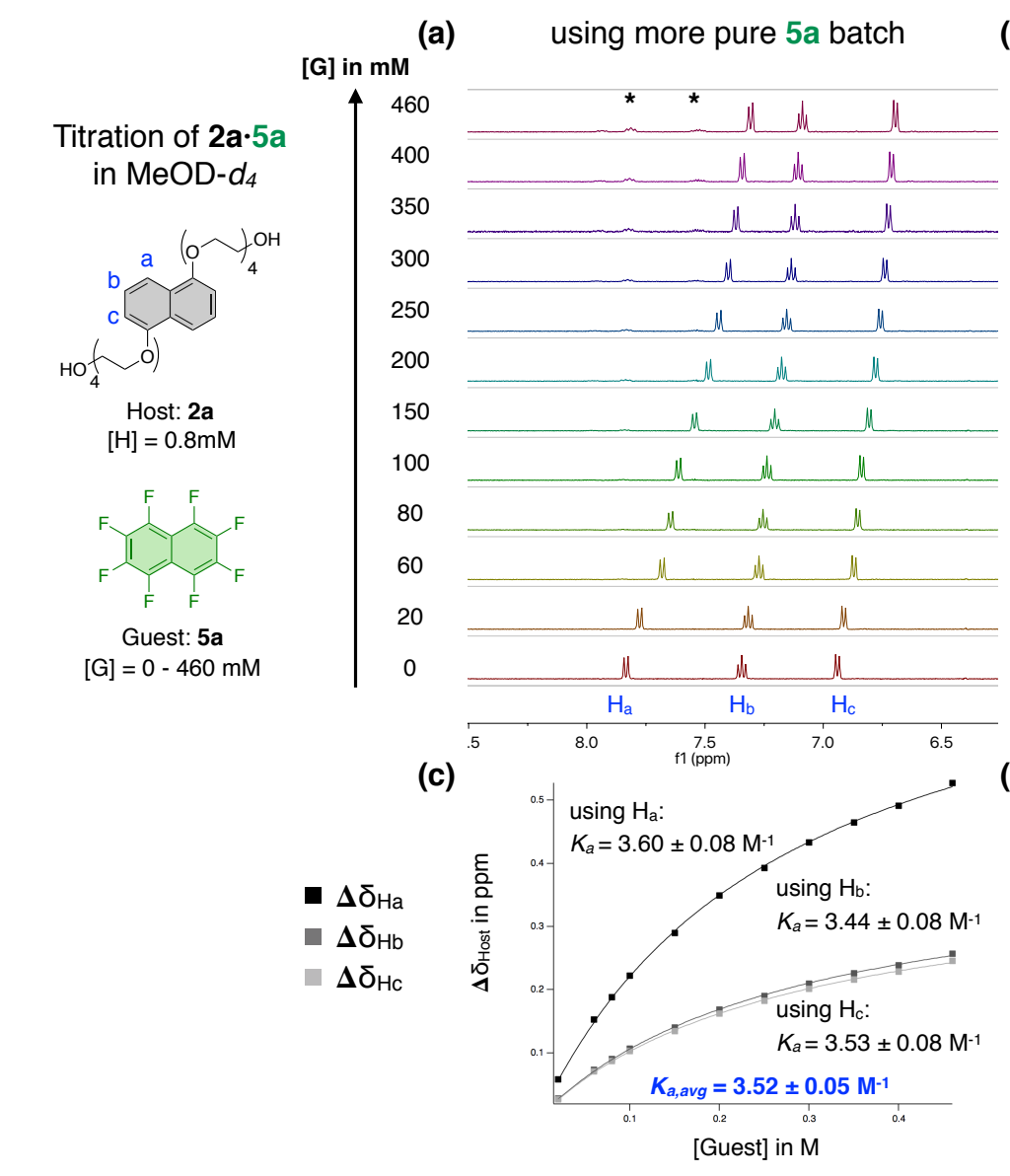

(b) using less pure 5a batch
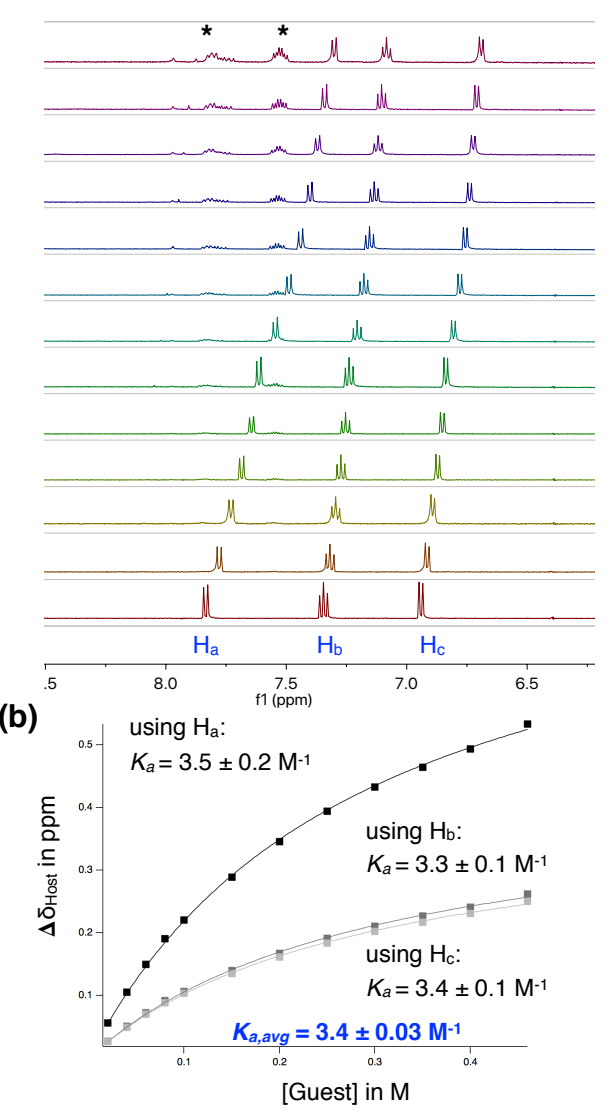

${ }^{*}=$ Partially hydrogenated impurities from octafluoronaphthalene $(\mathbf{5 a})$.

Figure S7. Impact of octafluoronapthalene impurity on $\mathbf{K}_{\mathbf{a}}$ measurements. Comparison of $K_{a}$ 's determined using more (a and $\mathbf{c}$ ) and less (b and $\mathbf{d})$ pure octafluoronaphthalene, 5a, for complex 2a·5a in MeOD- $d_{4}$. More pure octafluoronaphthalene, 5a, was purchased from Alfa Aesar and less pure 5a was purchased from SageChem.

Titration study using less pure 5a shows larger peaks of partially hydrogenated impurities between $7.5 \mathrm{ppm}$ and 8.0ppm (Fig S3b). Nonetheless, $K_{a}$ determined from each spectrum are similar (Fig S3c and S3d), indicating that the impurities do not play an important role in accurately measuring each $K_{a}$. 
a)
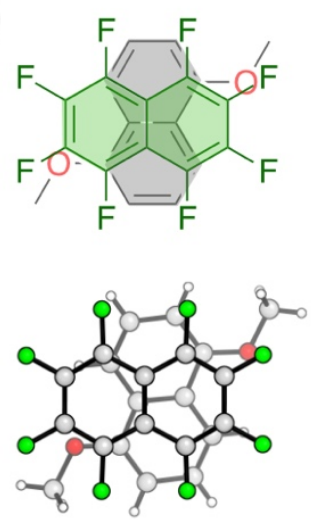

$2 b \cdot 5 a$
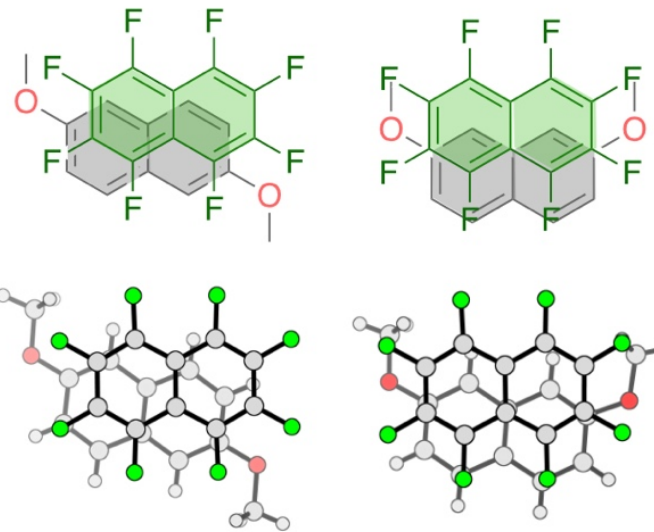

$2 b^{\prime} \cdot 5 a$

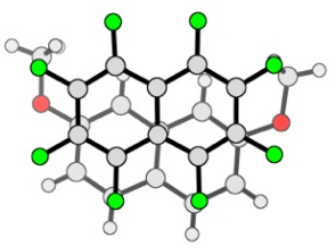

2b".5a

replace $\mathrm{OMe}$ with $\mathrm{H}$ (substituent effect)<smiles>Fc1c(F)c(F)c2c(c1F)-c1c(F)c(F)c(F)c(F)c1-2</smiles><smiles>Fc1c(F)c(F)c2c(F)c(F)c(F)c(F)c2c1F</smiles>

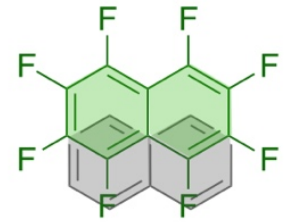

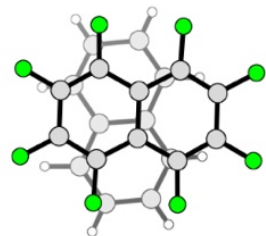

$\mathrm{C}_{10} \mathrm{H}_{8} \cdot 5 \mathrm{a}$

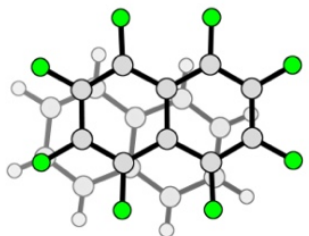

$\mathrm{C}_{10} \mathrm{H}_{8}{ }^{\prime} \cdot 5 \mathrm{a}$

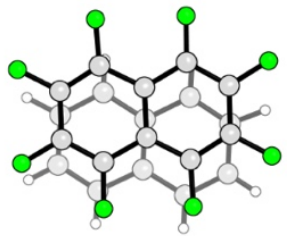

$\mathrm{C}_{10} \mathrm{H}_{8} " \cdot 5 \mathrm{a}$

b)

\begin{tabular}{c|cc|cccc} 
complex & $\Delta E_{\text {elec }}$ & $\Delta E_{\text {disp }}$ & $\Delta E_{\text {Pauli }}$ & $\Delta E_{\text {pol }}$ & $\Delta E_{\mathrm{CT}}$ & $\Delta E_{\text {int }}$ \\
\hline 2b. $\mathbf{C}_{10} \mathbf{D}_{8}$ & -13.8 & -16.7 & 25.5 & -0.8 & -0.4 & -6.2 \\
\hline $\mathbf{2 b} \cdot 5 \mathrm{a}$ & -12.5 & -15.8 & 17.4 & -1.3 & 0.0 & -12.2 \\
\hline $\mathbf{2 b} \cdot 5 \mathrm{a}$ & -16.9 & -17.4 & 23.2 & -1.5 & -0.1 & -12.7 \\
\hline $\mathbf{C}_{10} \mathrm{H}_{8} \cdot 5 \mathrm{a}$ & -15.3 & -15.6 & 22.0 & -1.3 & 0.0 & -10.2 \\
\hline $\mathbf{C}_{10} \mathrm{H}_{8} \cdot 5 \mathrm{a}$ & -15.1 & -15.1 & 21.6 & -1.3 & -0.1 & -10.4 \\
\hline $\mathbf{C}_{10} \mathrm{H}_{8} \cdot 5 \mathrm{5a}$ & -11.3 & -13.6 & 15.6 & -1.0 & 0.2 & -10.2
\end{tabular}

Figure S8. Additional computational analysis on substituent effects using 3 crystal structures, 2b-5a, 2,6dimethoxynaphthalene $\cdot 5 \mathrm{a}^{\mathrm{a}}$, and 2,7-dimethoxynaphthalene $\cdot 5 \mathrm{a}^{\mathrm{a}}$. (a) Scheme of computational analysis on substituent effect using truncated crystal structures. (b) Energy decomposition analysis (2nd generation ALMO-EDA) on the co-crystal structures and truncated computed structures. Electronic total energies are computed using 2nd generation ALMO-EDA with B97M-V and revPBE functionals and def2-svpd basis set. ${ }^{a}=$ crystal structures adapted from reference 10. 


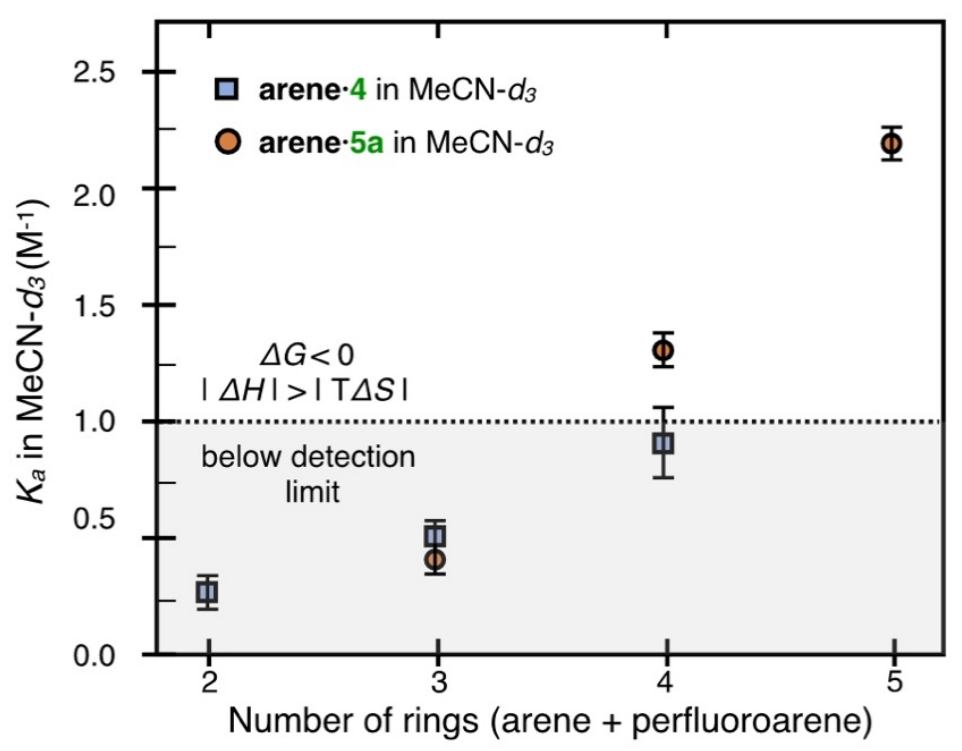

Figure S9. Additional experimental analysis on ring size effects. Experimental $K_{a}$ measurements of 6 differently sized arene-perfluoroarene complexes in $\mathrm{MeCN}-d_{3} . K_{a}$ 's are plotted against number of combined arene and perfluoroarene rings.. Dotted line indicates when enthalpy overcomes entropic penalty $\left(K_{a}=1 \mathrm{M}^{-1}\right.$ and $\Delta G=0 \mathrm{kcal}$ $\mathrm{mol}^{-1}$ ). Gray shaded area represents $K_{a}$ below 1 , which is the NMR detection limit. 


\section{Table of all $K_{a}$ data}

Table S1. Summary of all titration $\left(K_{a}\right)$ data obtained from NMR titration for arene-perfluoroarene, arene-arene, and fluoroarene-fluoroarene dimers. All $K_{a}$ 's are obtained in duplicate. ${ }^{a}=$ only $\mathrm{H}_{\mathrm{a}}$ (most downfield shifted aromatic proton) was used as other aromatic protons had overall small shift change $(<0.005 \mathrm{ppm}) .{ }^{\mathrm{b}}=$ only used aromatic proton signals that did not overlap with residual protonated aromatic guest.

\begin{tabular}{|c|c|c|c|c|c|}
\hline Entry & Complex & Solvent & $\begin{array}{l}K_{a}\left(\mathbf{M}^{-1}\right) \\
\text { Trial \#1 } \\
\end{array}$ & $\begin{array}{l}K_{a}\left(\mathbf{M}^{-1}\right) \\
\text { Trial \#2 } \\
\end{array}$ & $\begin{array}{l}K_{a}\left(\mathbf{M}^{-1}\right) \\
\text { average }\end{array}$ \\
\hline 1 & \multirow{2}{*}{$1 a \cdot 4$} & $\mathrm{CD}_{3} \mathrm{CN}$ & $0.3 \pm 0.1$ & $0.2 \pm 0.1$ & $0.3 \pm 0.1$ \\
\hline 2 & & $\mathrm{CD}_{3} \mathrm{OD}$ & $0.3 \pm 0.1$ & $0.2 \pm 0.1$ & $0.3 \pm 0.1$ \\
\hline 3 & \multirow{2}{*}{$2 a \cdot 4$} & $\mathrm{CD}_{3} \mathrm{CN}$ & $0.4 \pm 0.1$ & $0.6 \pm 0.1$ & $0.5 \pm 0.1$ \\
\hline 4 & & $\mathrm{CD}_{3} \mathrm{OD}$ & $0.4 \pm 0.1$ & $0.6 \pm 0.1$ & $0.5 \pm 0.1$ \\
\hline 5 & \multirow{5}{*}{$3 a \cdot 4$} & $\mathrm{CDCl}_{3}$ & $0.6 \pm 0.1$ & $0.6 \pm 0.3$ & $0.6 \pm 0.1$ \\
\hline 6 & & $\mathrm{CD}_{3} \mathrm{CN}$ & $0.7 \pm 0.1$ & $1.1 \pm 0.2$ & $0.9 \pm 0.2$ \\
\hline 7 & & DMSO- $d_{6}$ & $1.1 \pm 0.3$ & $1.1 \pm 0.3$ & $1.1 \pm 0.3$ \\
\hline 8 & & $\mathrm{CD}_{3} \mathrm{OD}$ & $1.4 \pm 0.1$ & $1.0 \pm 0.2$ & $1.2 \pm 0.2$ \\
\hline 9 & & 3:1 $\mathrm{CD}_{3} \mathrm{OD}: \mathrm{D}_{2} \mathrm{O}$ & $1.5 \pm 0.4$ & $1.4 \pm 0.1$ & $1.4 \pm 0.1$ \\
\hline 10 & \multirow{2}{*}{$1 a \cdot 5 a$} & $\mathrm{CD}_{3} \mathrm{CN}$ & $0.5 \pm 0.1$ & $0.3 \pm 0.1$ & $0.4 \pm 0.1$ \\
\hline 11 & & $\mathrm{CD}_{3} \mathrm{OD}$ & $0.7 \pm 0.1$ & $0.6 \pm 0.1$ & $0.7 \pm 0.1$ \\
\hline 12 & \multirow{2}{*}{$2 a \cdot 5 a$} & $\mathrm{CD}_{3} \mathrm{CN}$ & $1.3 \pm 0.1$ & $1.3 \pm 0.1$ & $1.3 \pm 0.1$ \\
\hline 13 & & $\mathrm{CD}_{3} \mathrm{OD}$ & $3.4 \pm 0.1$ & $3.5 \pm 0.1$ & $3.5 \pm 0.1$ \\
\hline 14 & \multirow{5}{*}{$3 a \cdot 5 a$} & $\mathrm{CDCl}_{3}$ & $1.3 \pm 0.2$ & $1.3 \pm 0.2$ & $1.3 \pm 0.1$ \\
\hline 15 & & $\mathrm{CD}_{3} \mathrm{CN}$ & $2.1 \pm 0.1$ & $2.3 \pm 0.1$ & $2.2 \pm 0.1$ \\
\hline 16 & & DMSO- $d_{6}$ & $3.1 \pm 0.2$ & $2.6 \pm 0.1$ & $2.8 \pm 0.2$ \\
\hline 17 & & $\mathrm{CD}_{3} \mathrm{OD}$ & $4.7 \pm 0.2$ & $4.6 \pm 0.5$ & $4.7 \pm 0.1$ \\
\hline 18 & & 3:1 $\mathrm{CD}_{3} \mathrm{OD}: \mathrm{D}_{2} \mathrm{O}$ & $21.5 \pm 0.5$ & $22.0 \pm 0.5$ & $21.8 \pm 0.3$ \\
\hline 19 & \multirow{5}{*}{$3 a \cdot 5 b$} & 3:1 CD $\mathrm{CD}_{3} \mathrm{OD}: \mathrm{D}_{2} \mathrm{O}$ & $19 \pm 3$ & $18 \pm 1$ & $18.9 \pm 0.5$ \\
\hline 20 & & 1:1 $\mathrm{CD}_{3} \mathrm{OD}: \mathrm{D}_{2} \mathrm{O}$ & $30 \pm 4$ & $31 \pm 3$ & $30.0 \pm 0.5$ \\
\hline 21 & & $\mathrm{D}_{2} \mathrm{O}$ & $48 \pm 5$ & $50 \pm 3$ & $49 \pm 1$ \\
\hline 22 & & $\mathrm{D}_{2} \mathrm{O}+\mathrm{KCl}$ & $190 \pm 80^{a}$ & $100 \pm 40^{\mathrm{a}}$ & $120 \pm 50^{a}$ \\
\hline 23 & & $\begin{array}{c}\mathrm{D}_{2} \mathrm{O}+ \\
\mathrm{K}_{2} \mathrm{HPO}_{4} / \mathrm{K}_{3} \mathrm{HPO}_{4} \\
\end{array}$ & $5300 \pm 700^{\mathrm{a}}$ & $8600 \pm 2000^{a}$ & $6000 \pm 1000^{a}$ \\
\hline 24 & $3 a \cdot C_{6} D_{6}$ & $\mathrm{CD}_{3} \mathrm{OD}$ & $0.4 \pm 0.1^{b}$ & $0.3 \pm 0.1^{\mathrm{b}}$ & $0.4 \pm 0.1^{b}$ \\
\hline 25 & $3 \mathrm{a} \cdot \mathrm{C}_{10} \mathrm{D}_{8}$ & $\mathrm{CD}_{3} \mathrm{OD}$ & $1.0 \pm 0.1^{\mathrm{b}}$ & $0.7 \pm 0.1^{\mathrm{b}}$ & $0.8 \pm 0.2^{b}$ \\
\hline 26 & $2 \mathrm{a} \cdot \mathrm{C}_{\mathbf{1 0}} \mathrm{D}_{\mathbf{8}}$ & $\mathrm{CD}_{3} \mathrm{OD}$ & $0.2 \pm 0.1$ & $0.3 \pm 0.1$ & $0.3 \pm 0.1$ \\
\hline
\end{tabular}




\section{5. ${ }^{1} \mathrm{H}$ and ${ }^{19}$ F NMR Titration Data}

\section{$\underline{5.1 K_{a}}$ data for complex $1 \mathrm{a} \cdot \mathbf{4}$}

\section{Complex 1a.4 in $\mathrm{MeCN}-d_{3}$}

\section{[G] in $\mathrm{mM}$}

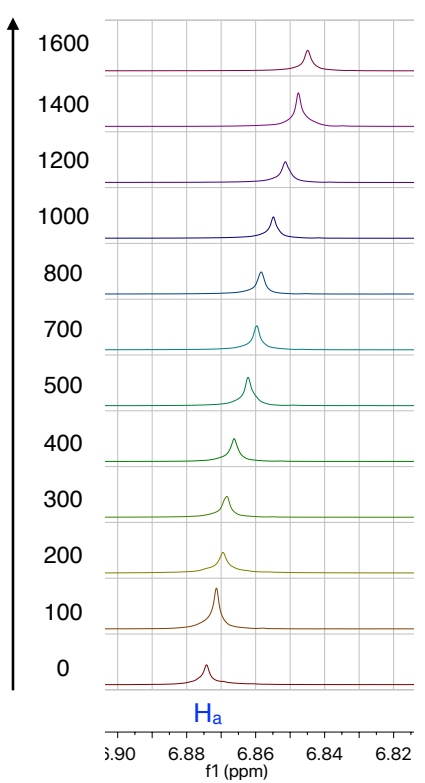

\section{Complex 1a·4 in MeOD-d $d_{4}$}

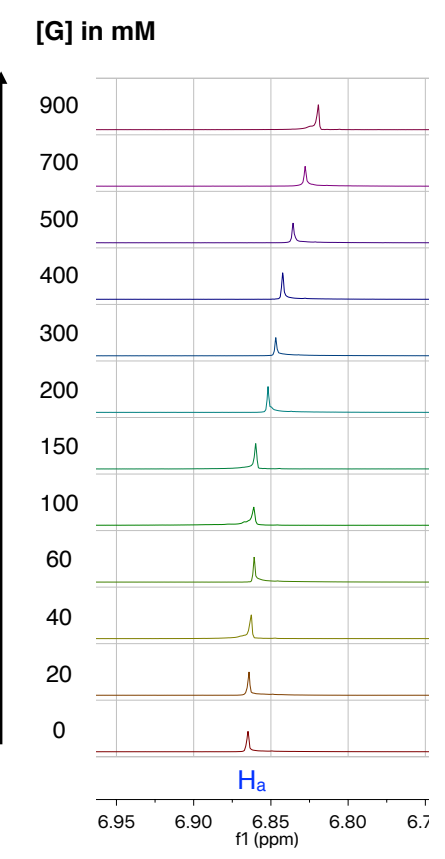

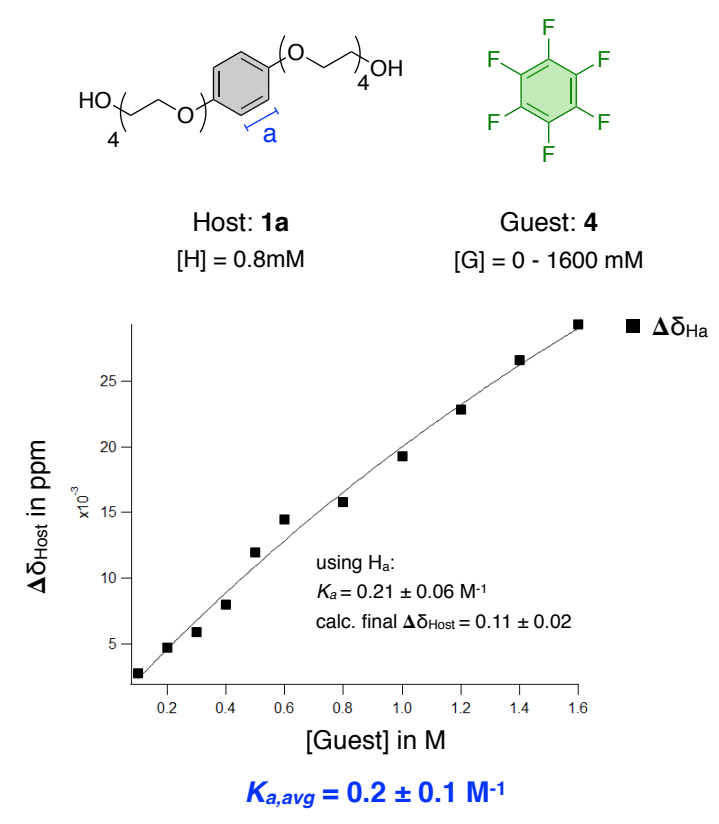




\section{$\underline{5.2} K_{a}$ data for complex $2 \mathrm{a} \cdot \mathbf{4}$}

\section{Complex 2a-4 in $\mathrm{MeCN}-d_{3}$}

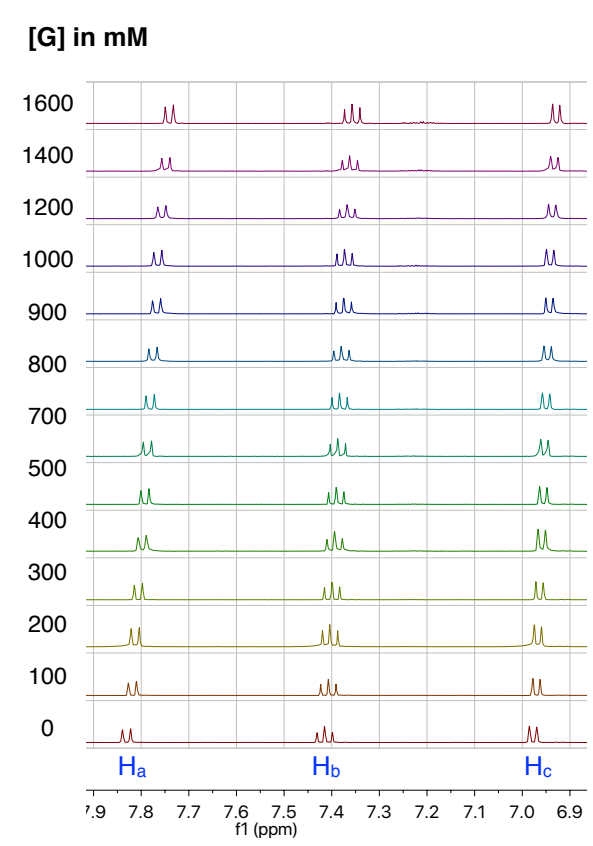

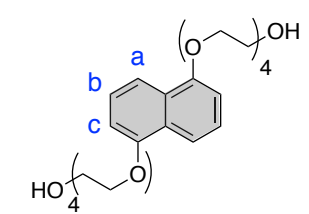

Host: $\mathbf{2 a}$

$[\mathrm{H}]=0.8 \mathrm{mM}$

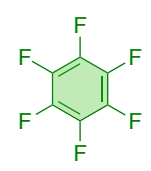

Guest: 4

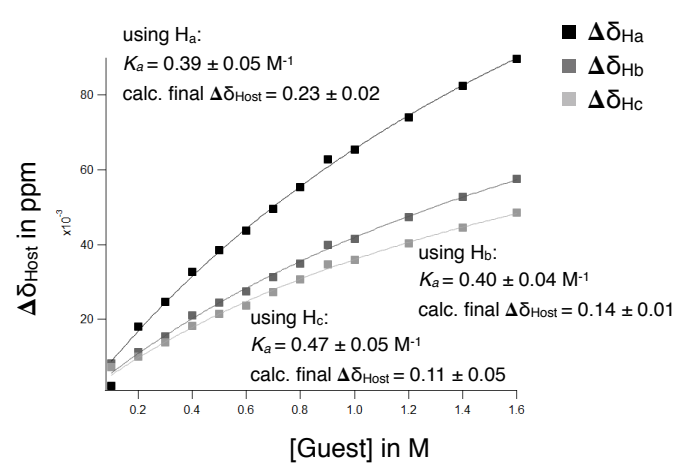

$K_{a, a v g}=0.4 \pm 0.1 \mathrm{M}^{-1}$

\section{Complex 2a-4 in MeOD- $d_{4}$}

[G] in $\mathrm{mM}$

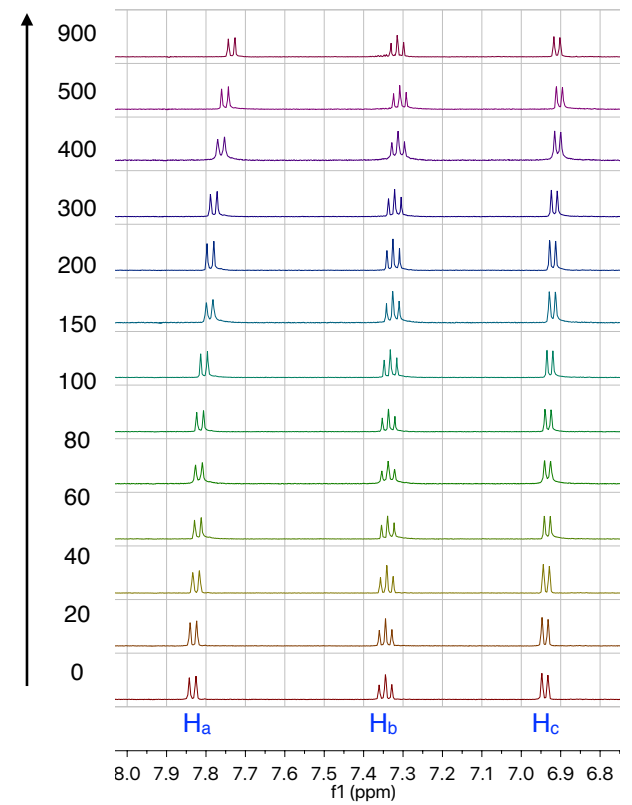

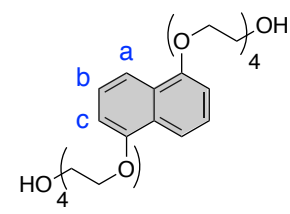

Host: 2a

$[\mathrm{H}]=0.8 \mathrm{mM}$

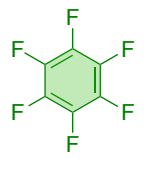

Guest: 4

$[\mathrm{G}]=0-900 \mathrm{mM}$

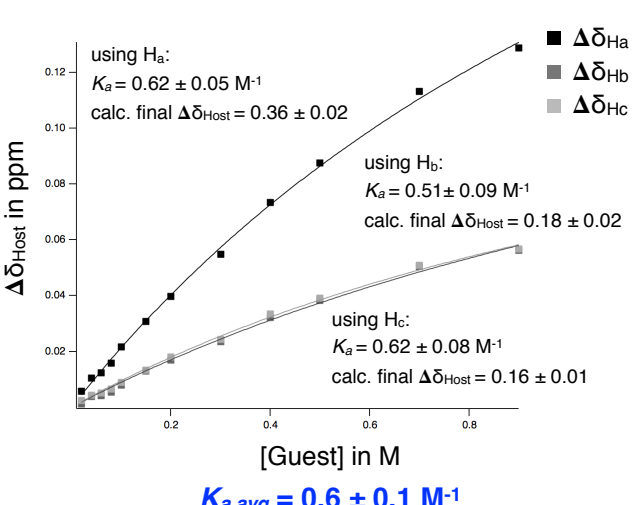

$K_{a, a v g}=0.6 \pm 0.1 \mathrm{M}^{-1}$ 


\section{$\underline{5.3 K_{a}}$ data for complex $\mathbf{3 a} \cdot \mathbf{4}$}

\section{Complex 3a・4 in $\mathrm{CDCl}_{3}$}

\section{[G] in $\mathrm{mM}$}

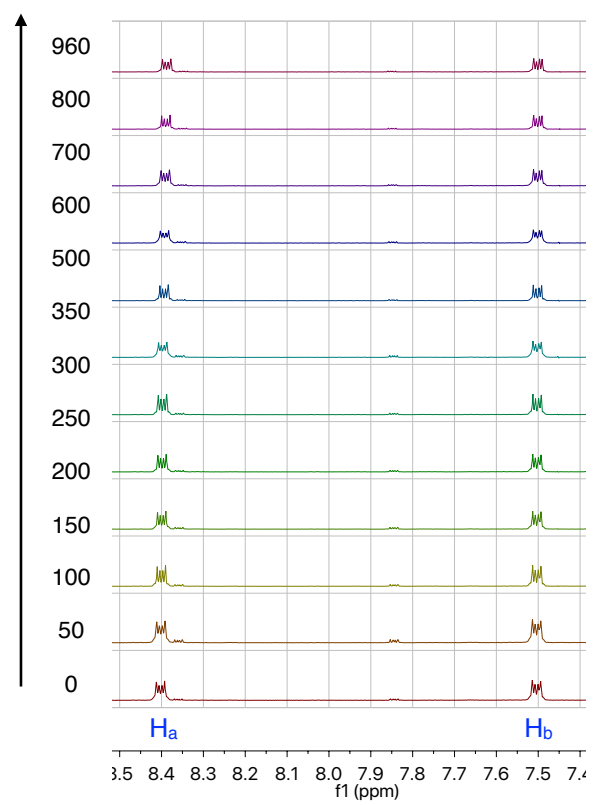

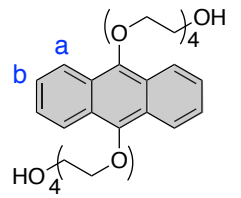

Host: $\mathbf{3 a}$

$[\mathrm{H}]=0.8 \mathrm{mM}$

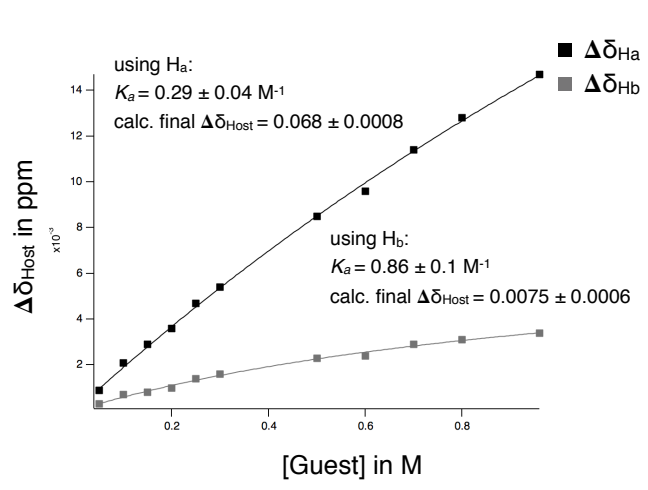

$K_{a, a v g}=0.6 \pm 0.3 \mathrm{M}^{-1}$

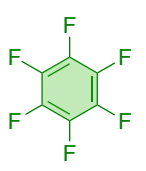

Guest: 4

[G] $=0-960 \mathrm{mM}$

\section{Complex 3a·4 in DMSO- $d_{6}$}

\section{[G] in $\mathrm{mM}$}

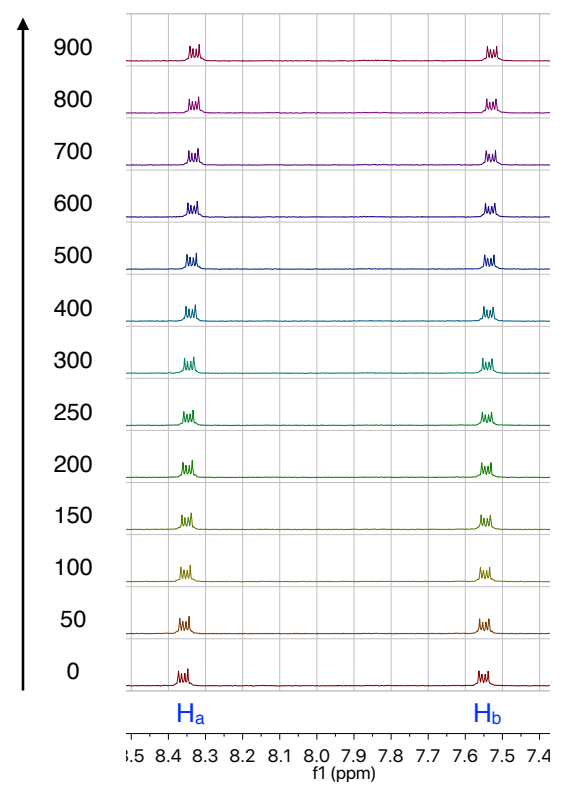

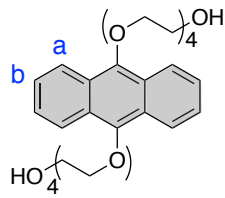

Host: $\mathbf{3 a}$

$[\mathrm{H}]=0.8 \mathrm{mM}$

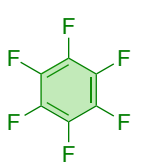

Guest: 4

$[\mathrm{G}]=0-960 \mathrm{mM}$

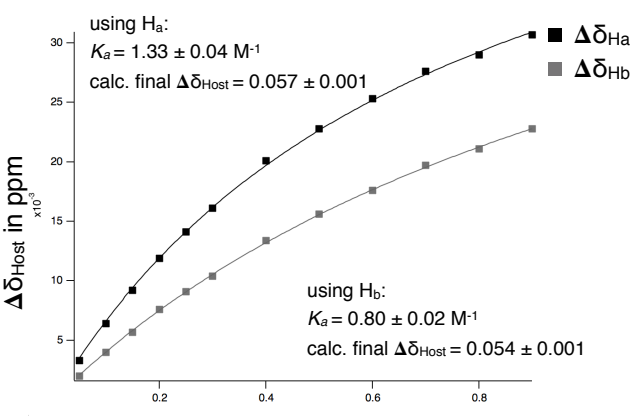

[Guest] in M

$K_{a, a v g}=1.1 \pm 0.3 \mathrm{M}^{-1}$ 


\section{Complex 3a.4 in MeCN-d $d_{3}$}

\section{[G] in $\mathrm{mM}$}

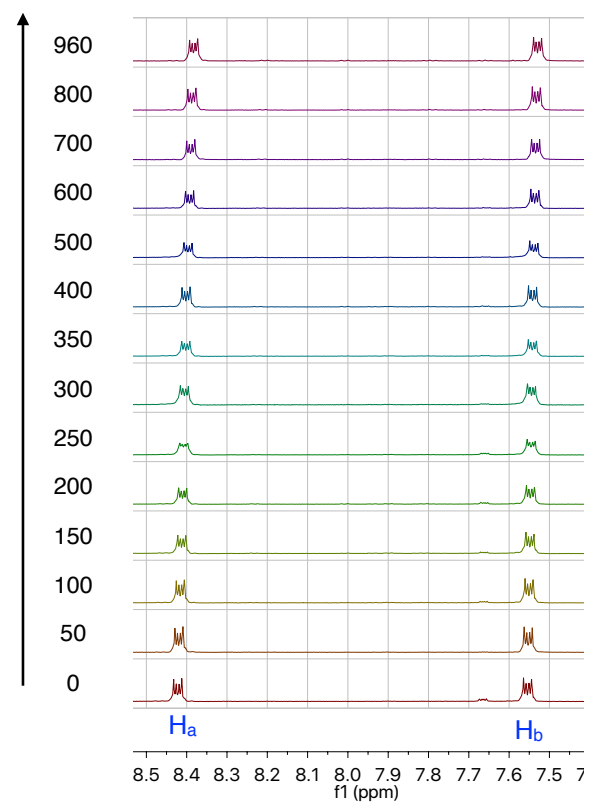

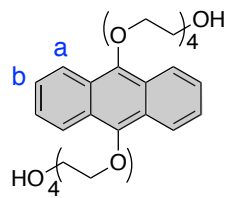

Host: 3a

$[\mathrm{H}]=0.8 \mathrm{mM}$

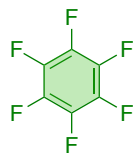

Guest: 4

[G] $=0-960 \mathrm{mM}$

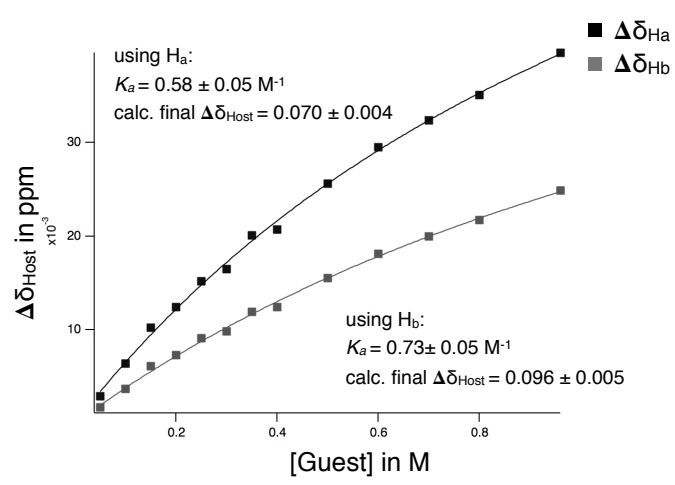

$K_{a, a v g}=0.7 \pm 0.1 \mathrm{M}^{-1}$

\section{Complex $\mathbf{3 a} \cdot \mathbf{4}$ in MeOD- $d_{4}$}

\section{[G] in $\mathrm{mM}$}

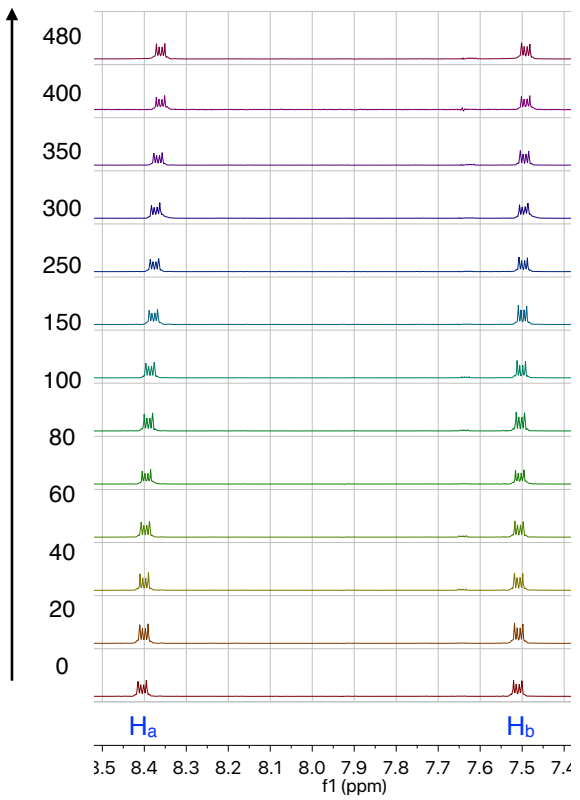

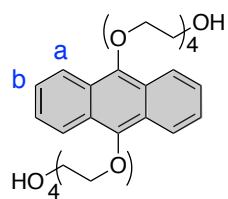

Host: 3a

$[\mathrm{H}]=0.8 \mathrm{mM}$

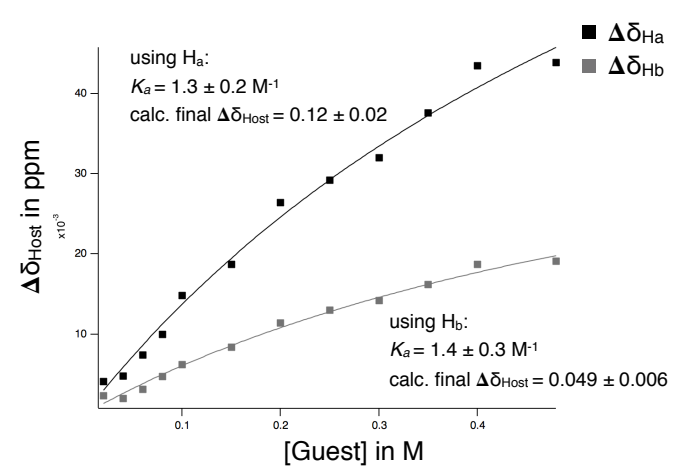

$K_{a, a v g}=1.4 \pm 0.1 \mathrm{M}^{-1}$ 


\section{Complex 3a-4 in 3:1 MeOD- $d_{4}: \mathrm{D}_{2} \mathrm{O}$}

\section{[G] in $\mathrm{mM}$}

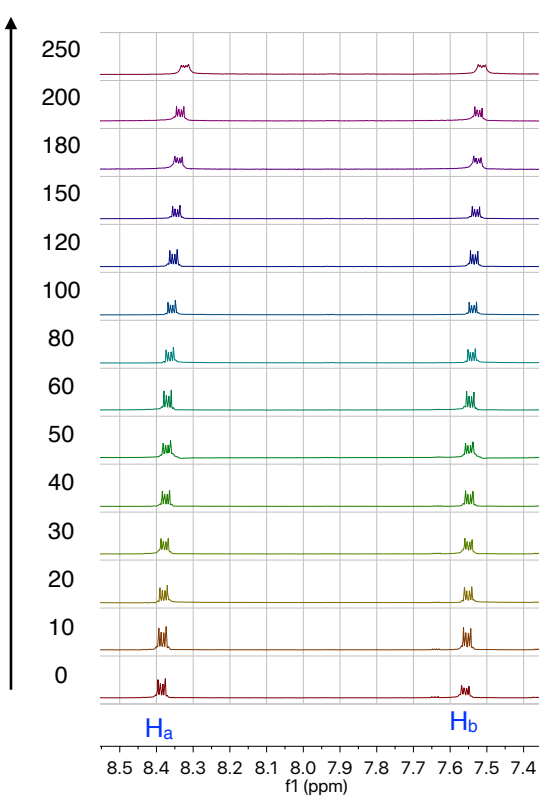

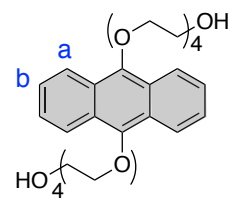

Host: 3a

$[\mathrm{H}]=0.8 \mathrm{mM}$

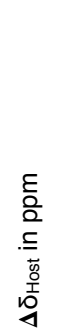




\section{$\underline{5.4 K_{a}} \underline{\text { data for complex } 1 \mathrm{a} \cdot \mathbf{5 a}}$}

\section{Complex 1a.5a in $\mathrm{MeCN}-d_{3}$}

\section{[G] in $\mathrm{mM}$}

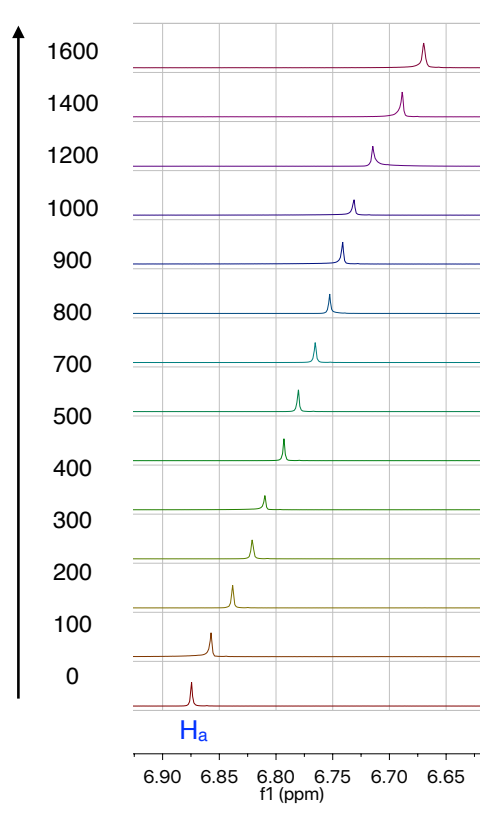

\section{Complex 1a·5a in MeOD- $d_{4}$}

\section{[G] in $\mathrm{mM}$}

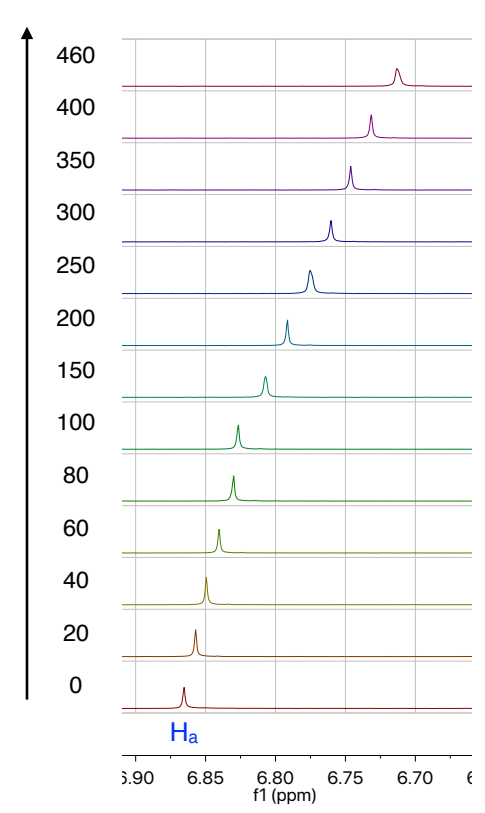<smiles>CC(C)(O)COc1ccc(OCC(C)(O)O)cc1</smiles>

Host: 1a

$[\mathrm{H}]=0.8 \mathrm{mM}$

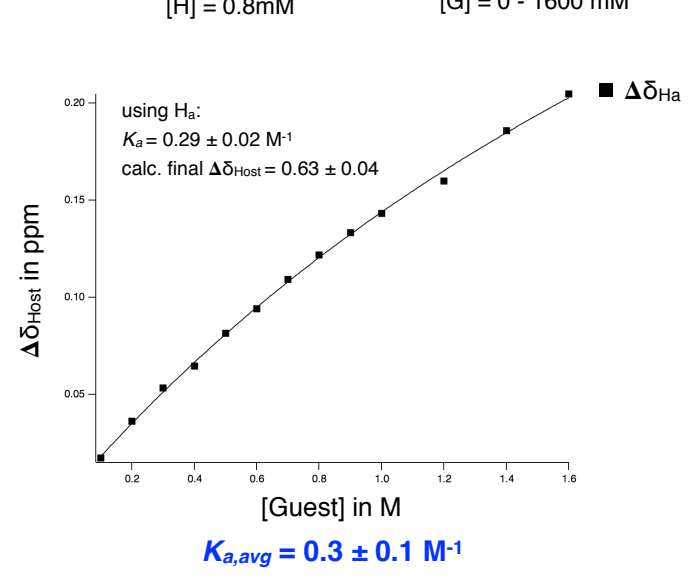

$K_{a, a v g}=0.3 \pm 0.1 \mathrm{M}^{-1}$

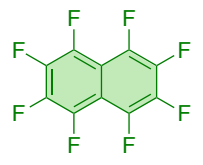

Guest: $\mathbf{5 a}$

$[G]=0-1600 \mathrm{mM}$ 


\section{$\underline{5.5} K_{a}$ data for complex $2 \mathbf{a} \cdot \mathbf{5 a}$}

\section{Complex 2a-5a in $\mathrm{MeCN}-d_{3}$}

[G] in $\mathrm{mM}$

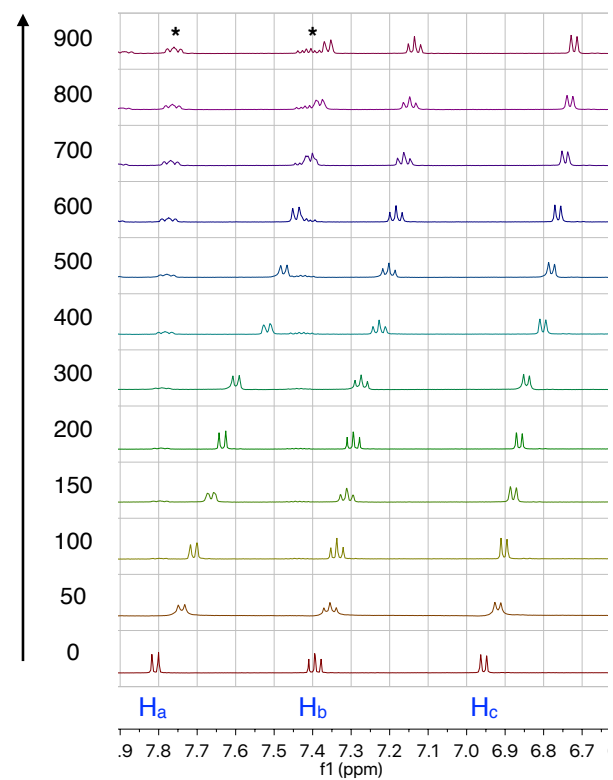

* = Partially hydrogenated impurities from octafluoronaphthalene (5a).

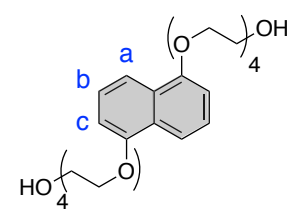

Host: 2a

$[\mathrm{H}]=0.8 \mathrm{mM}$

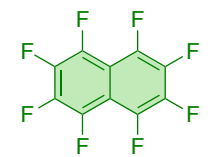

Guest: 5 a

[G] $=0-960 \mathrm{mM}$

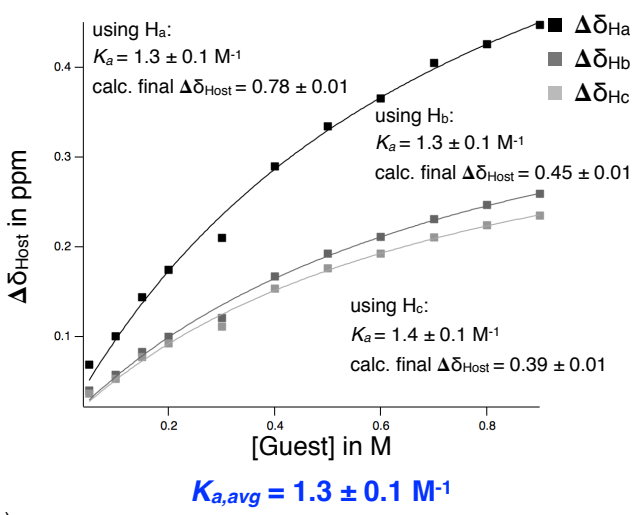

Complex 2a-5a in MeOD- $d_{4}$

[G] in $\mathrm{mM}$

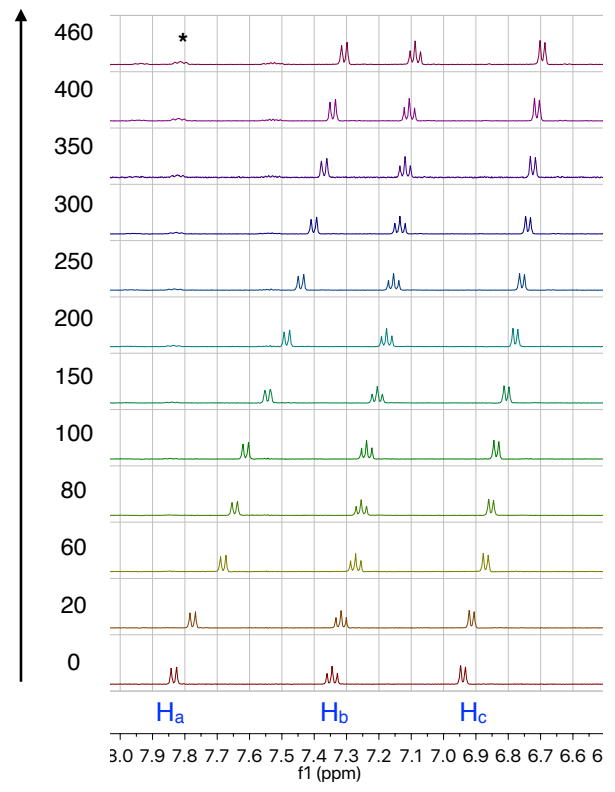

* $=$ Partially hydrogenated impurities from octafluoronaphthalene $\mathbf{( 5 a}$.

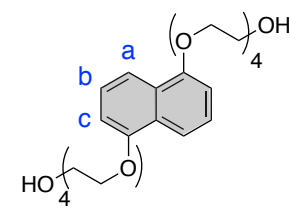

Host: 2a

$[\mathrm{H}]=0.8 \mathrm{mM}$

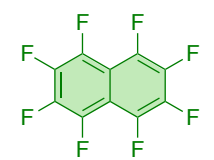

Guest: 5 a

[G] $=0-460 \mathrm{mM}$

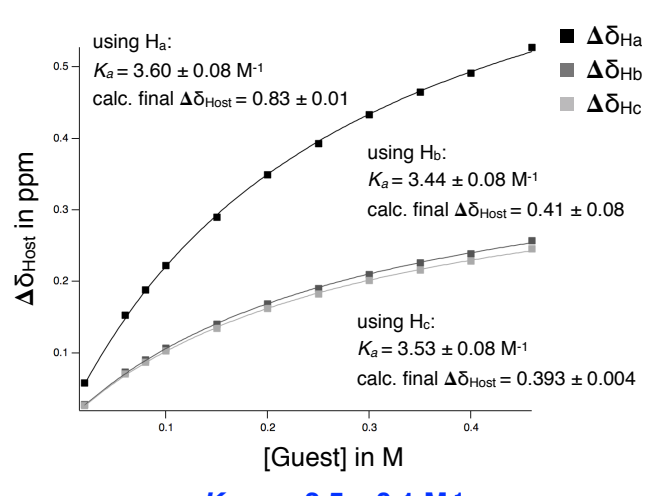

$K_{a, a v g}=3.5 \pm 0.1 \mathrm{M}^{-1}$ 


\section{$\underline{\mathbf{5 . 6} K_{a}}$ data for complex $\mathbf{3 a} \cdot \mathbf{5 a}$}

\section{Complex $\mathbf{3 a} \cdot \mathbf{5 a}$ in $\mathrm{CDCl}_{3}$}

[G] in $\mathrm{mM}$

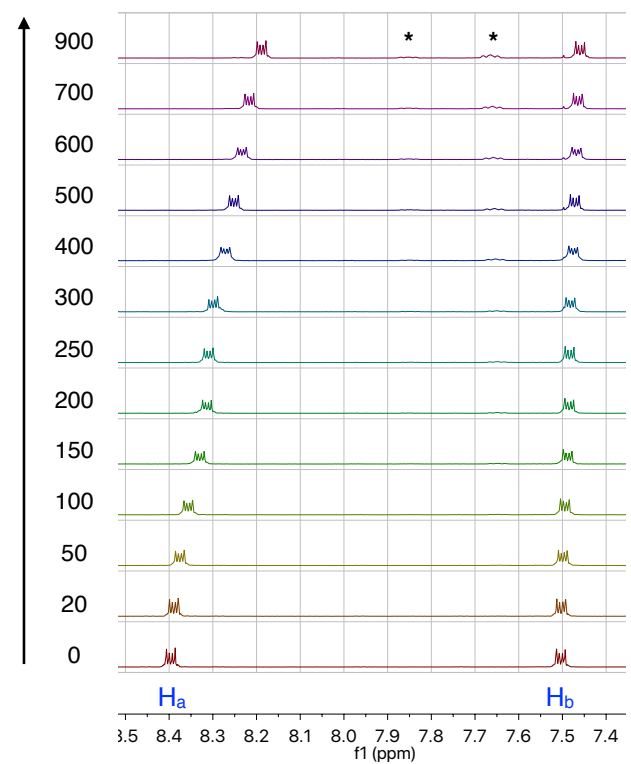

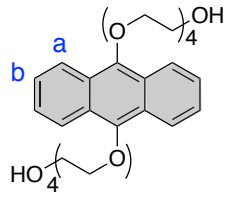

Host: 3a $[\mathrm{H}]=0.8 \mathrm{mM}$

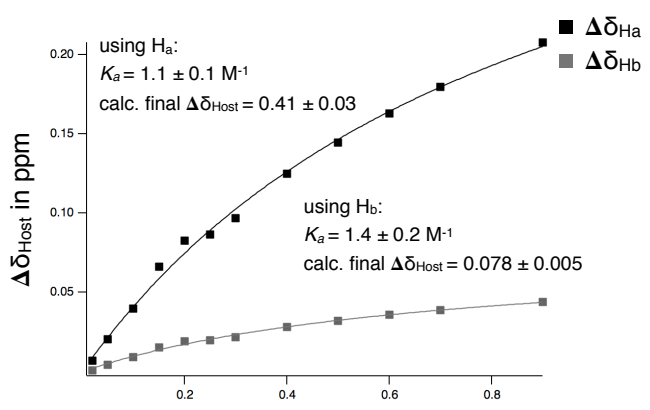

[Guest] in $\mathrm{M}$

$K_{a, a v g}=1.3 \pm 0.2 \mathrm{M}^{-1}$

* = Partially hydrogenated impurities from octafluoronaphthalene (5a).

\section{Complex 3a·5a in DMSO- $d_{6}$}

[G] in $\mathrm{mM}$

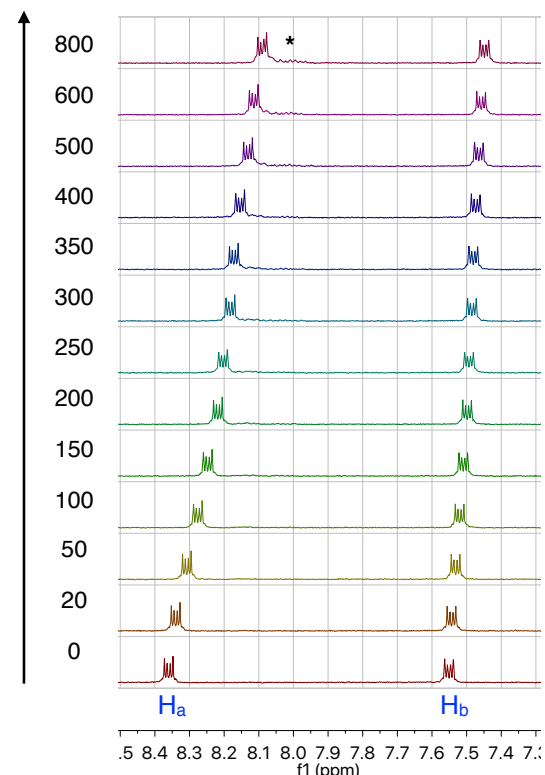

* = Partially hydrogenated impurities from octafluoronaphthalene (5a).

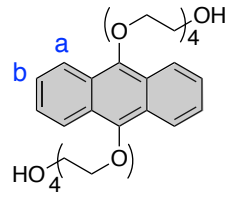

Host: $3 \mathbf{a}$ $[\mathrm{H}]=0.8 \mathrm{mM}$

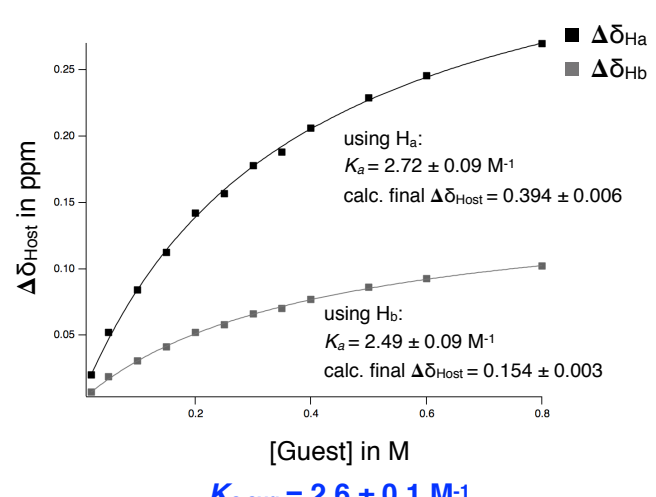




\section{Complex 3a.5a in $\mathrm{MeCN}-d_{3}$}

\section{[G] in $\mathrm{mM}$}

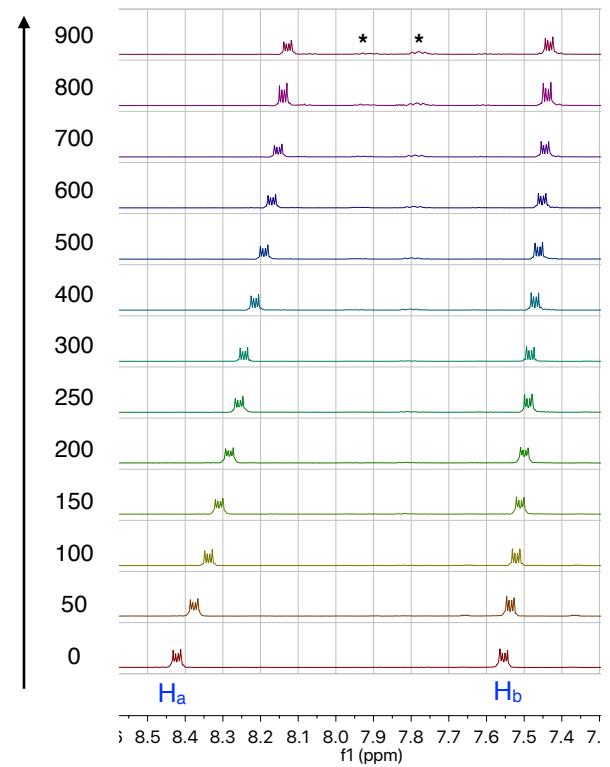

* = Partially hydrogenated impurities from octafluoronaphthalene (5a)

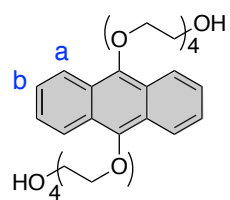

Host: $3 a$

$[\mathrm{H}]=0.8 \mathrm{mM}$

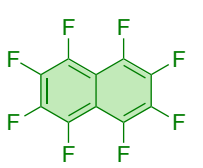

Guest: $5 \mathbf{a}$ $[\mathrm{G}]=0-900 \mathrm{mM}$

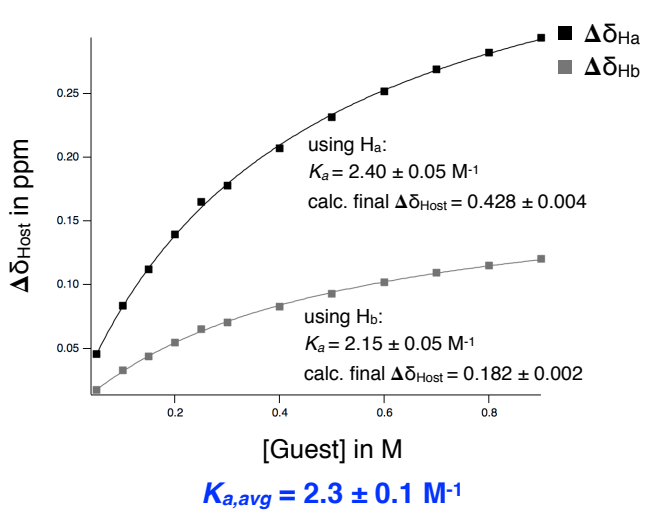

\section{Complex 3a·5a in MeOD- $d_{4}$}

[G] in $\mathrm{mM}$

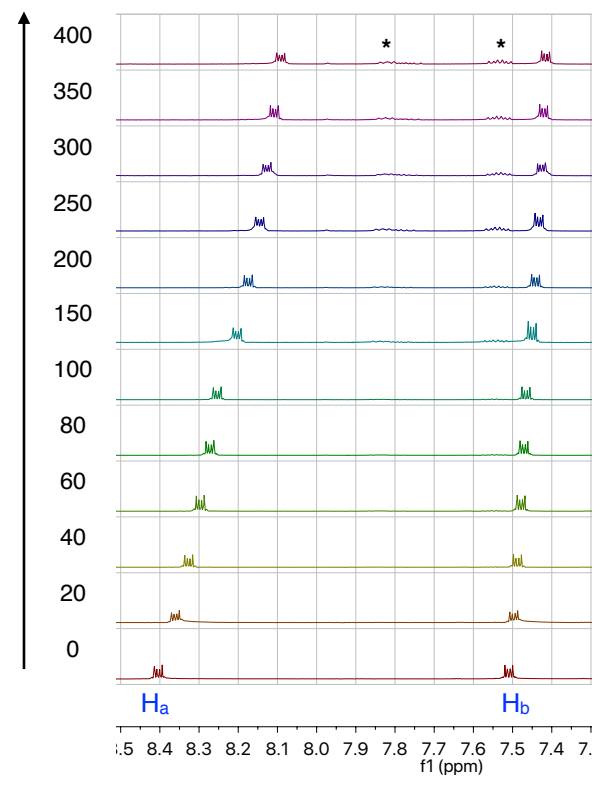

* = Partially hydrogenated impurities from octafluoronaphthalene (5a)

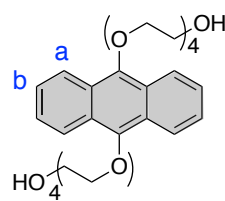

Host: $3 a$

$[\mathrm{H}]=0.8 \mathrm{mM}$

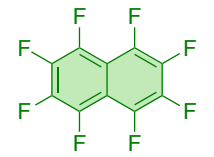

Guest: $5 \mathbf{a}$

$[\mathrm{G}]=0-400 \mathrm{mM}$

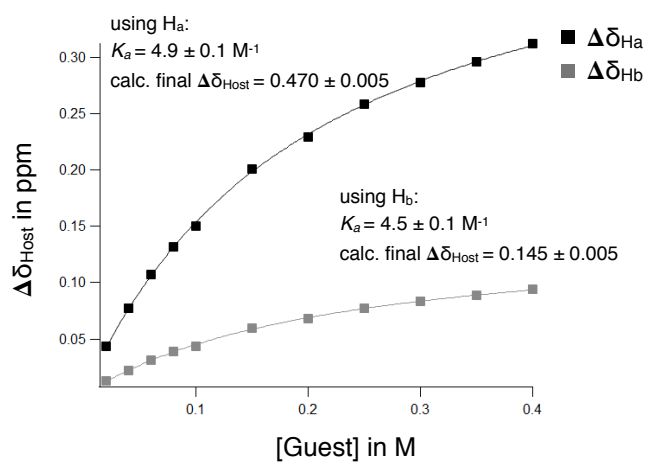

$K_{a, a v g}=4.7 \pm 0.1 \mathrm{M}^{-1}$ 
Complex 3a·5a in 3:1 MeOD- $d_{4}: \mathrm{D}_{2} \mathrm{O}$
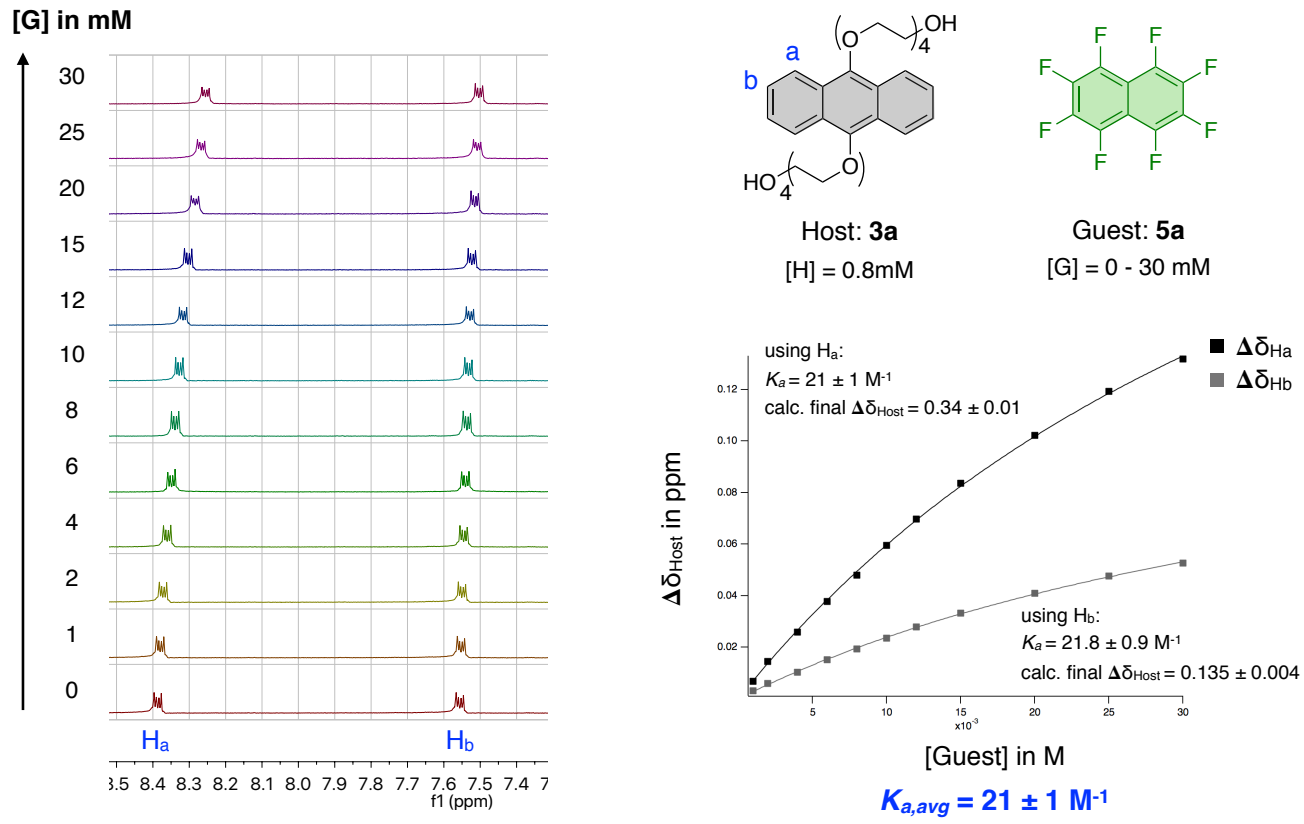


\section{$\underline{\mathbf{5 . 7} K_{a}} \underline{\text { data for complex } \mathbf{3 a} \cdot \mathbf{5 b}}$}

\section{Complex 3a·5b in 3:1 MeOD- $d_{4}: \mathrm{D}_{2} \mathrm{O}$}

\section{[G] in $\mathrm{mM}$}

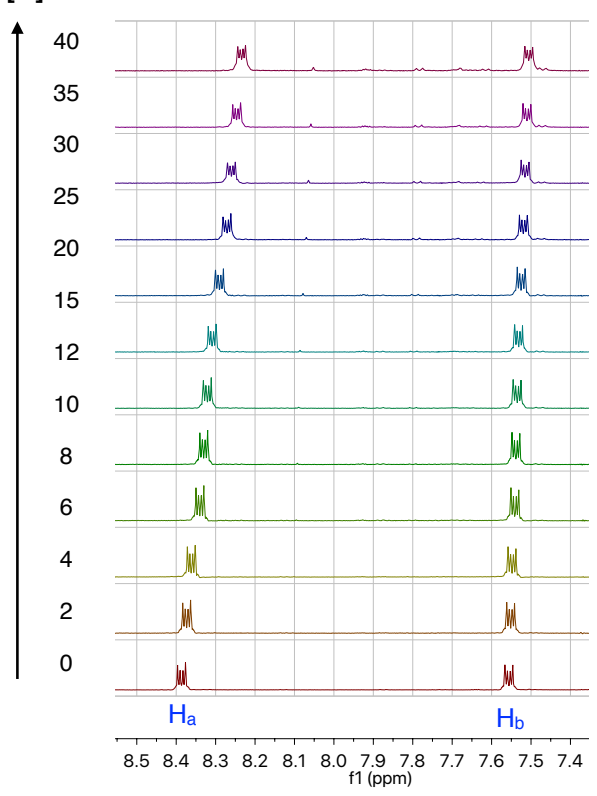

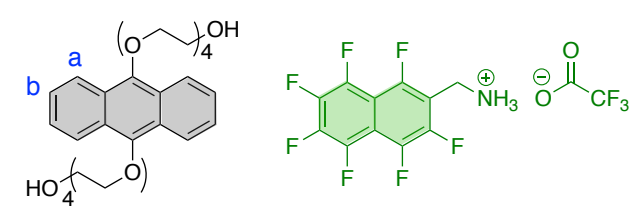

Host: 3a

$[\mathrm{H}]=0.8 \mathrm{mM}$

Guest: 5a

[G] $=0-40 \mathrm{mM}$

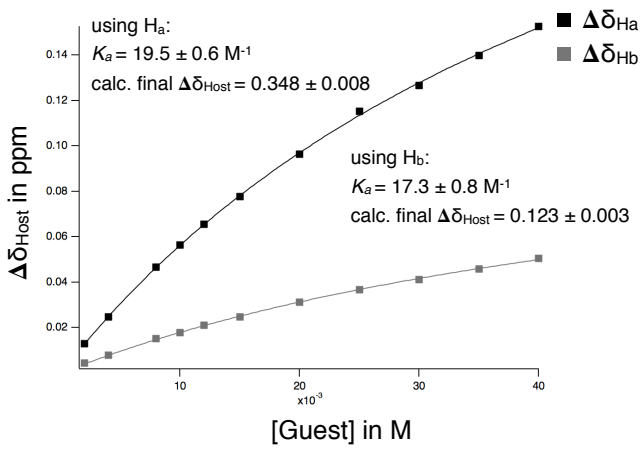

$K_{a, a v g}=18.4 \pm 0.7 \mathrm{M}^{-1}$

\section{Complex 3a-5b in 1:1 MeOD- $d_{4}: \mathrm{D}_{2} \mathrm{O}$}

\section{[G] in $\mathrm{mM}$}

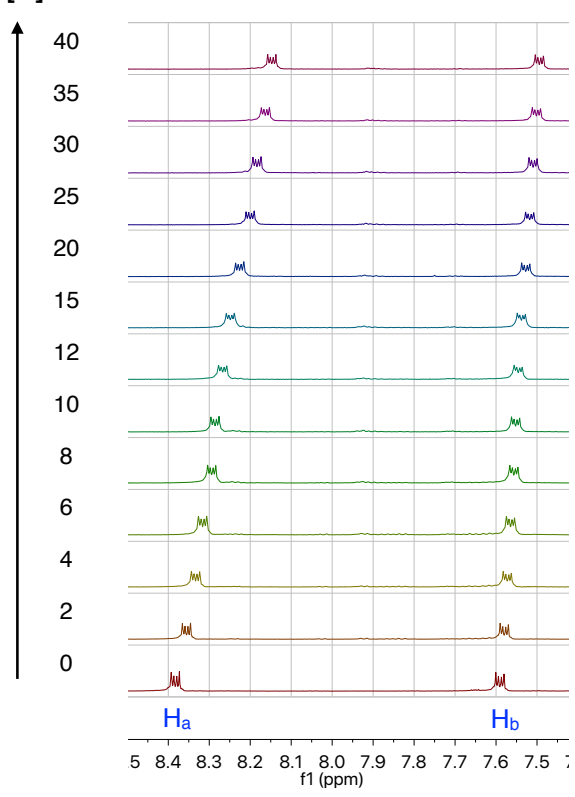

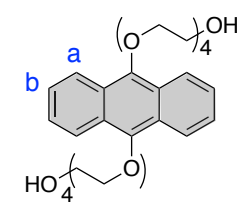

Host: $\mathbf{3 a}$

$[\mathrm{H}]=0.8 \mathrm{mM}$

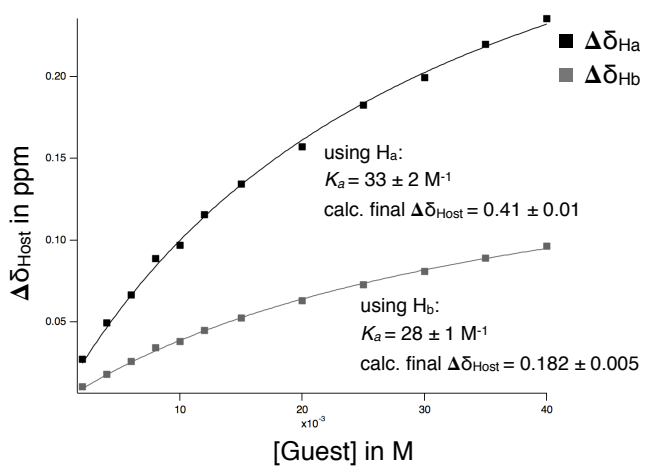

$K_{a, a v g}=31 \pm 3 \mathrm{M}^{-1}$

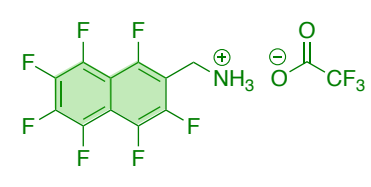

Guest: 5a

$[G]=0-40 \mathrm{mM}$

using $\mathrm{H}_{\mathrm{a}}$ :

$K_{a}=33 \pm 2 \mathrm{M}^{-1}$

using $\mathrm{H}_{\mathrm{b}}$

$K_{\mathrm{a}}=28 \pm 1 \mathrm{M}^{-1}$

calc. final $\Delta \delta_{\text {Host }}=0.182 \pm 0.005$ 


\section{Complex $3 a \cdot 5 b$ in $\mathrm{D}_{2} \mathrm{O}$}

[G] in $\mathrm{mM}$

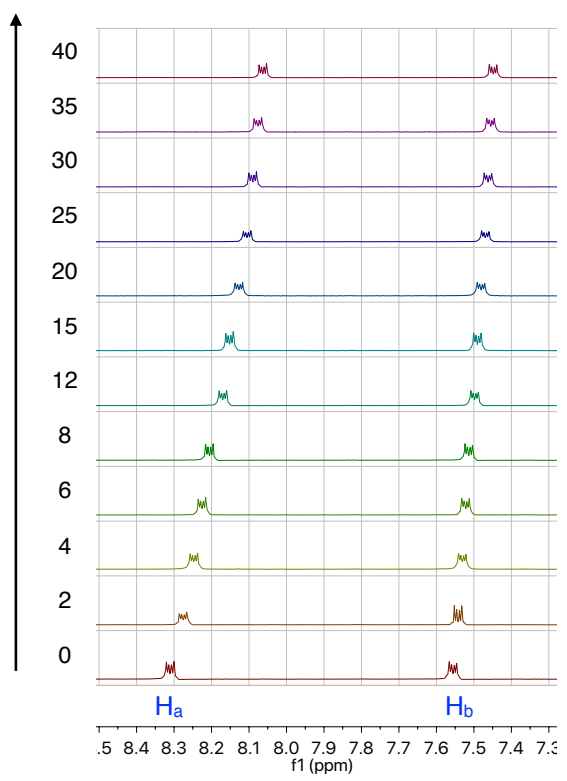

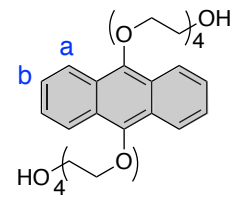

Host: $\mathbf{3 a}$

$[\mathrm{H}]=0.8 \mathrm{mM}$

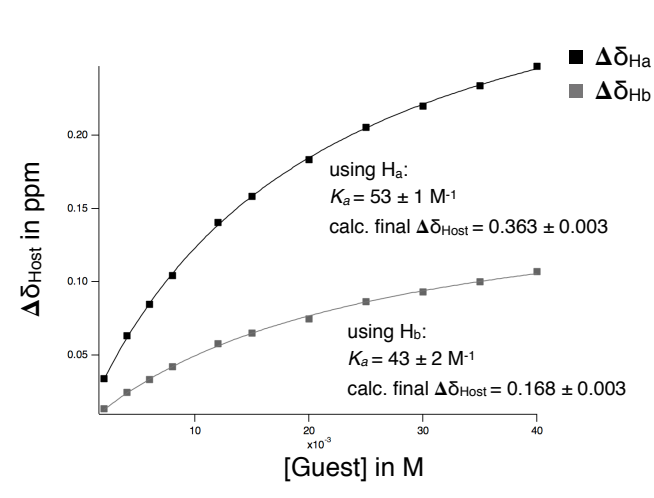

$K_{a, a v g}=48 \pm 1 \mathrm{M}^{-1}$

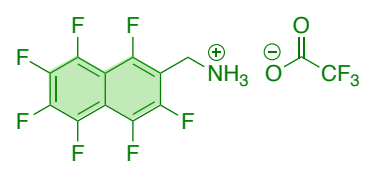

Guest: 5a

$[\mathrm{G}]=0-60 \mathrm{mM}$

using $\mathrm{H}_{\mathrm{a}}$

$K_{a}=53 \pm 1 \mathrm{M}$

using $\mathrm{H}_{\mathrm{b}}$ :

$K_{a}=43 \pm 2 \mathrm{M}-$

\section{Complex $\mathbf{3 a} \cdot \mathbf{5 b}$ in $\mathrm{D}_{2} \mathrm{O}$ with $\mathrm{KCl}$}

[G] in $\mathrm{mM}$

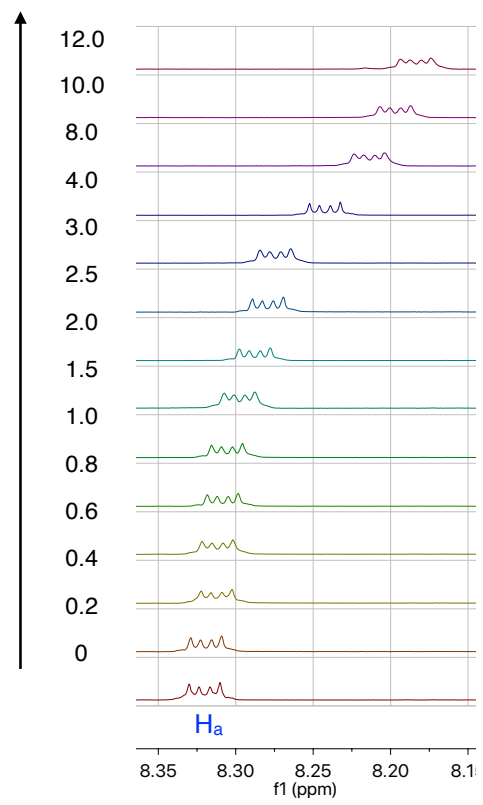

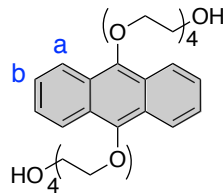

Host: $\mathbf{3 a}$

$[\mathrm{H}]=0.8 \mathrm{mM}$

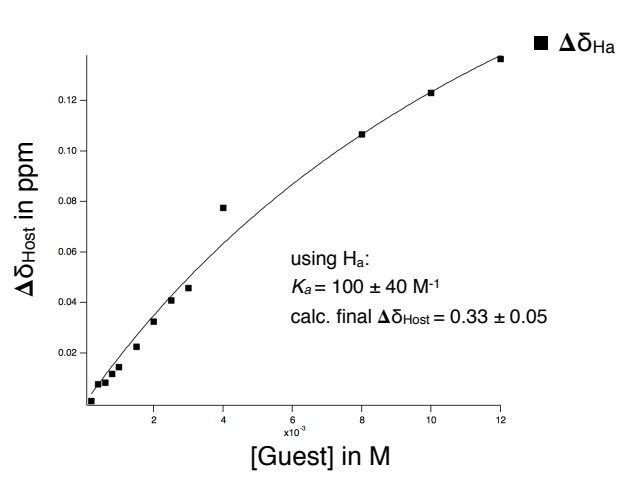




\section{Complex 3a·5b in $\mathrm{D}_{2} \mathrm{O}$ with $\mathrm{K}_{3} \mathrm{PO}_{4} / \mathrm{K}_{2} \mathrm{HPO}_{4}$}

[G] in $\mathrm{mM}$

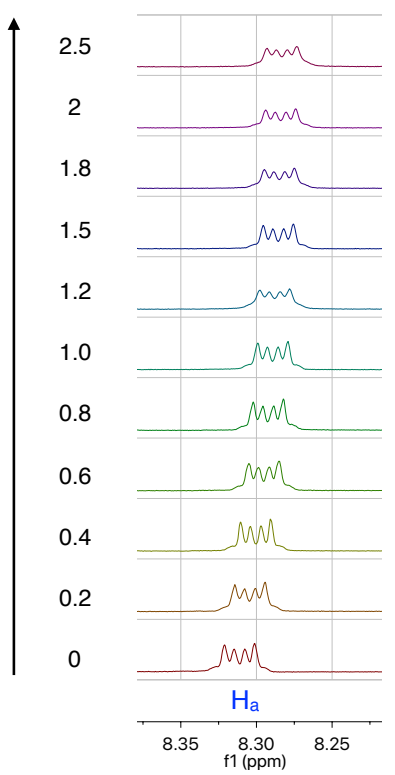

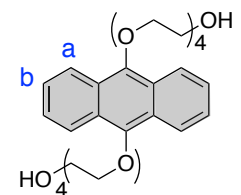

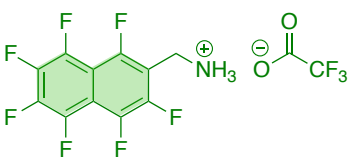

Host: $\mathbf{3 a}$

$[\mathrm{H}]=0.8 \mathrm{mM}$

Guest: $\mathbf{5 a}$

$[\mathrm{G}]=0-60 \mathrm{mM}$

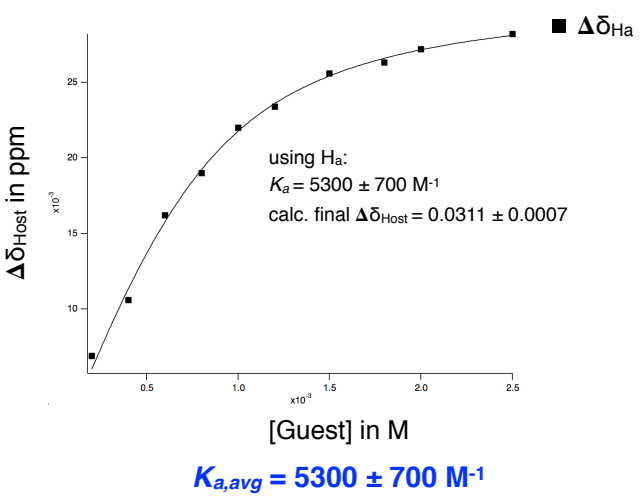




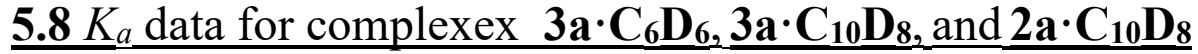

\section{Complex $3 \mathbf{a} \cdot \mathbf{B}-\boldsymbol{d}_{6}$ in MeOD- $\boldsymbol{d}_{4}$}

\section{[G] in $\mathrm{mM}$}

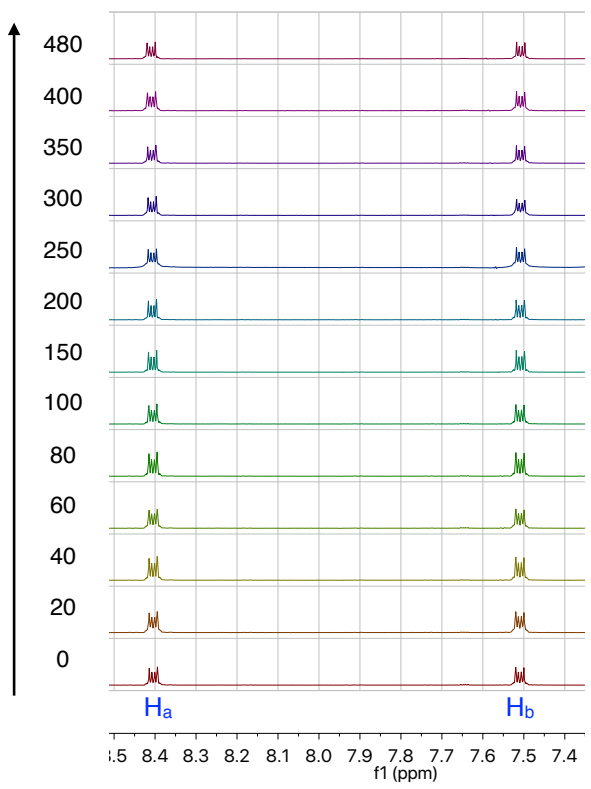

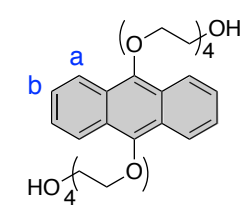

Host: $\mathbf{3 a}$

$[\mathrm{H}]=0.8 \mathrm{mM}$

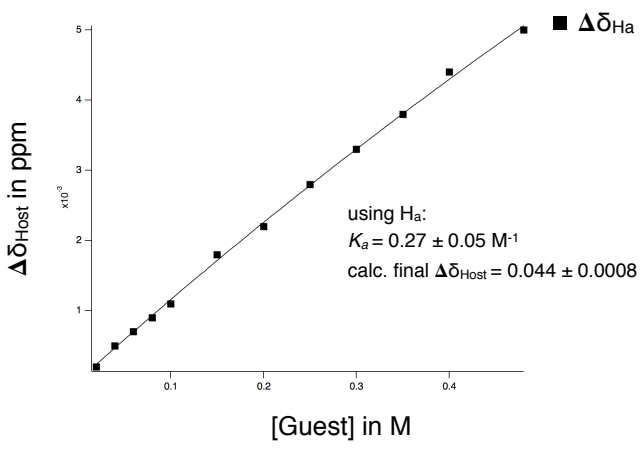

$K_{a, a v g}=0.3 \pm 0.1 \mathrm{M}^{-1}$

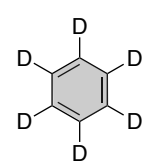

Guest: B- $\boldsymbol{d}_{\boldsymbol{6}}$

[G] $=0-60 \mathrm{mM}$

$K_{a}=0.27 \pm 0.05 \mathrm{M}^{-1}$

alc. final $\Delta \delta_{\text {Host }}=0.044 \pm 0.0008$
Complex 3a-N- $d_{8}$ in MeOD- $d_{4}$

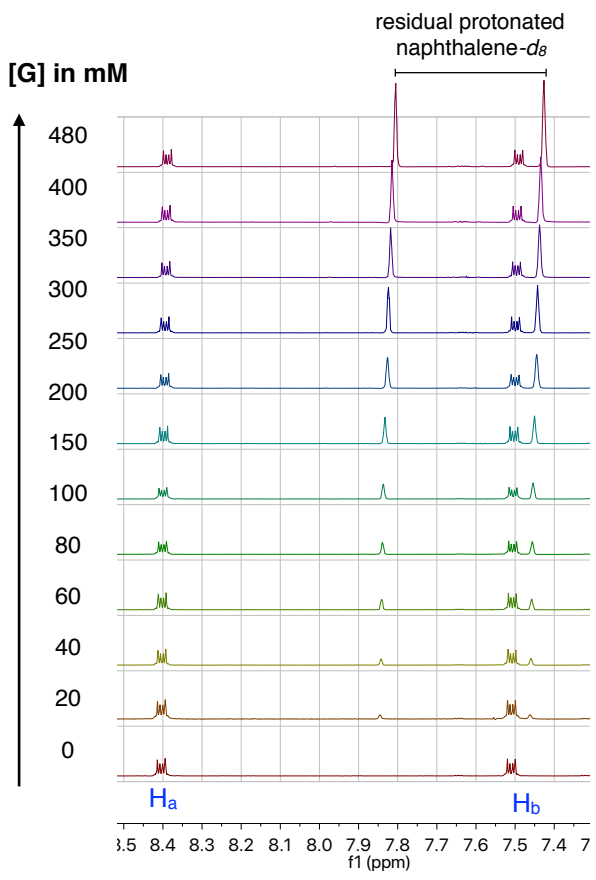

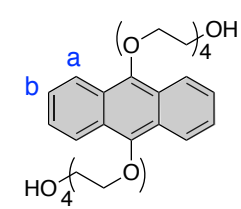

Host: $3 a$

$[\mathrm{H}]=0.8 \mathrm{mM}$

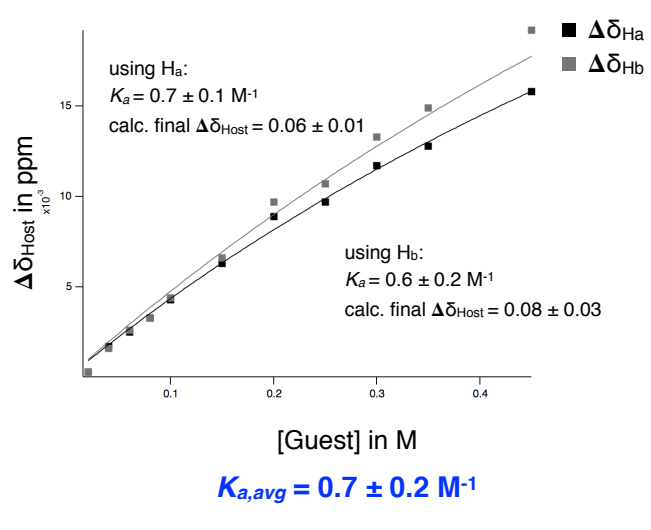




\section{Complex $\mathbf{2} \cdot \mathbf{N}-\boldsymbol{d}_{\boldsymbol{8}}$ in MeOD- $\boldsymbol{d}_{4}$}
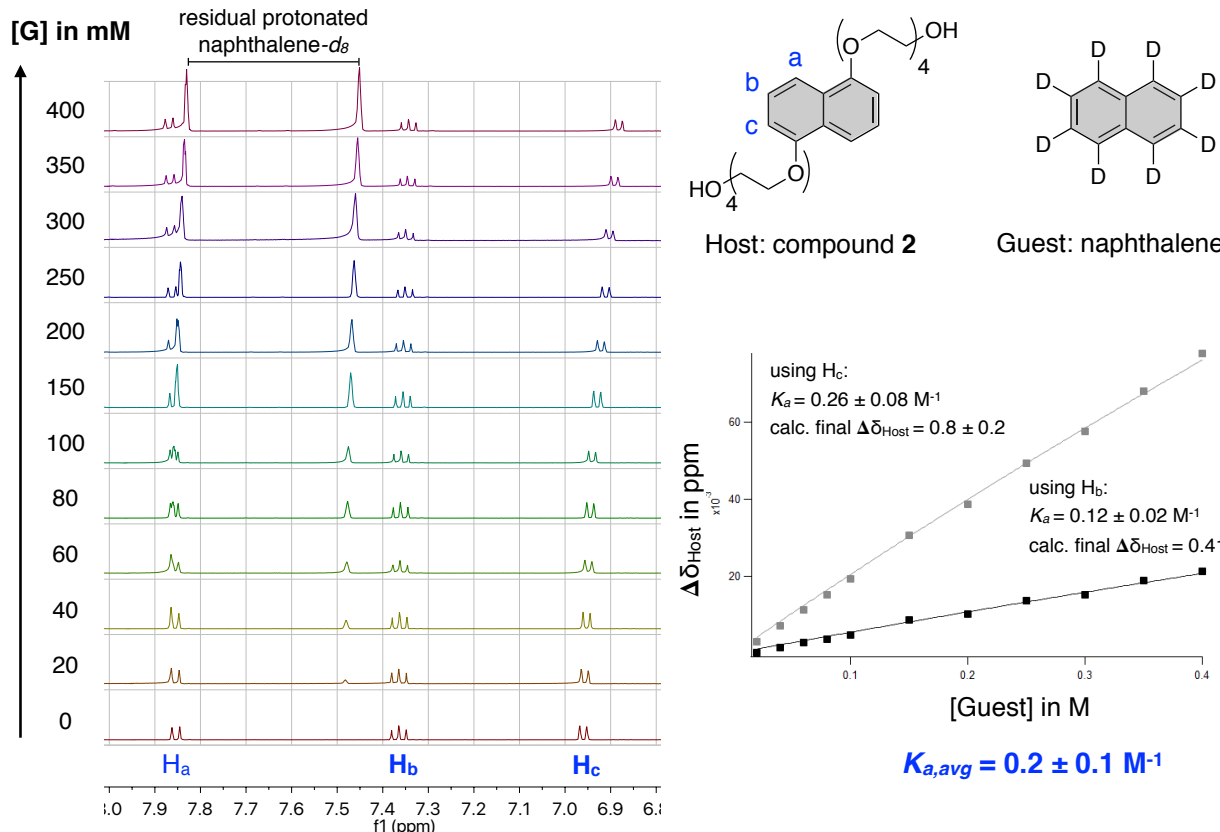

Host: compound 2

Guest: naphthalene- $d_{8}$

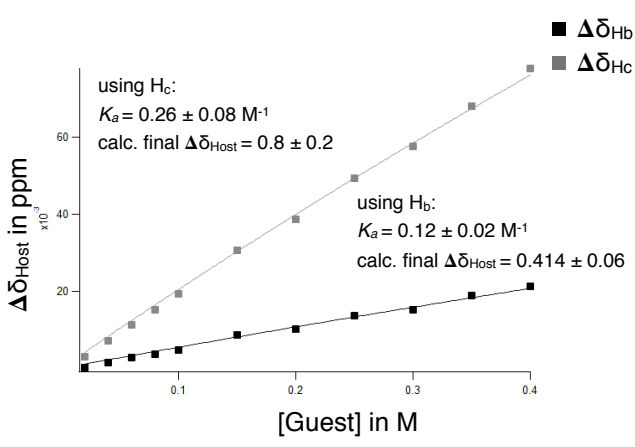

$K_{a, a v g}=0.2 \pm 0.1 \mathrm{M}^{-1}$

Only $H_{b}$ and $H_{c}$ are used for titration 


\section{Crystallographic Information}

\subsection{Crystal data and structure for complex $\mathbf{2 b} \cdot \mathbf{4}$ (CCDC number: 2060321)}
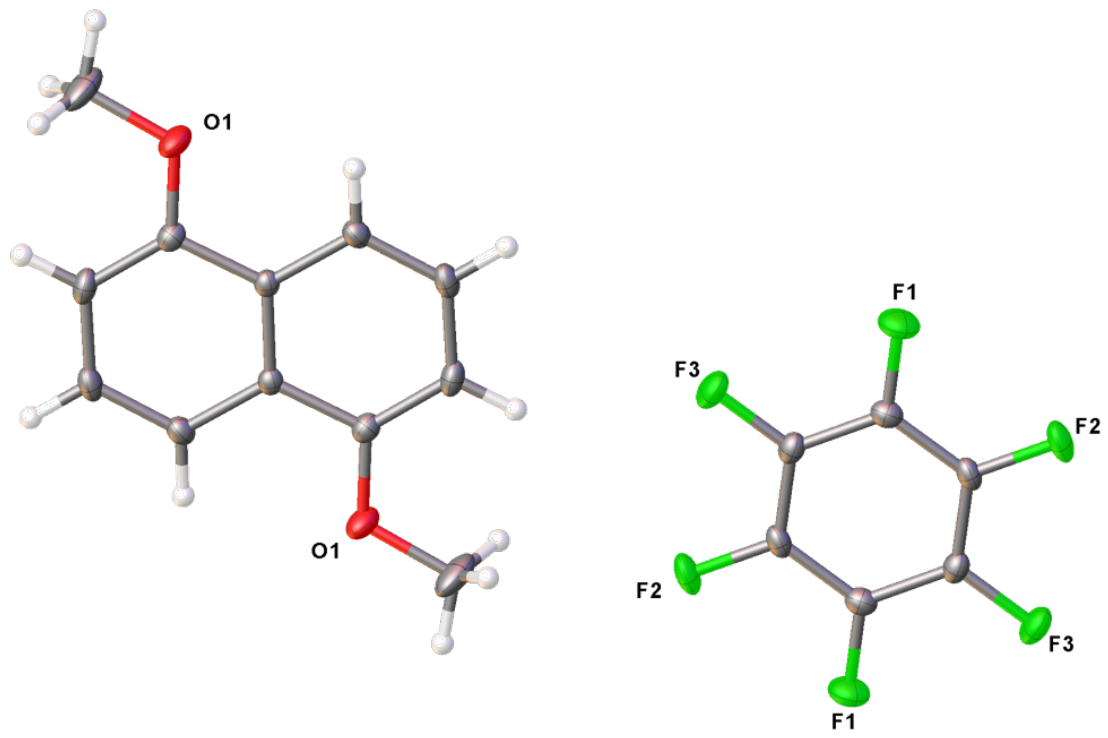

Table S2. Crystal data and structure refinement for complex $\mathbf{2 b} \cdot \mathbf{4}$.

Identification code

Empirical formula

Formula weight

Temperature

Wavelength

Crystal system

Space group

Unit cell dimensions

Volume

Z, Z'

Density (calculated)

Absorption coefficient

$\mathrm{F}(000)$

Crystal size

Theta range for data collection

Index ranges

Reflections collected

Independent reflections

Completeness to theta $=25.242^{\circ}$

Absorption correction

Max. and min. transmission

Refinement method

Data / restraints / parameters

Goodness-of-fit on $\mathrm{F}^{2}$

Final $\mathrm{R}$ indices [I $>2$ sigma(I)]

$\mathrm{R}$ indices (all data)

Extinction coefficient

Largest diff. peak and hole

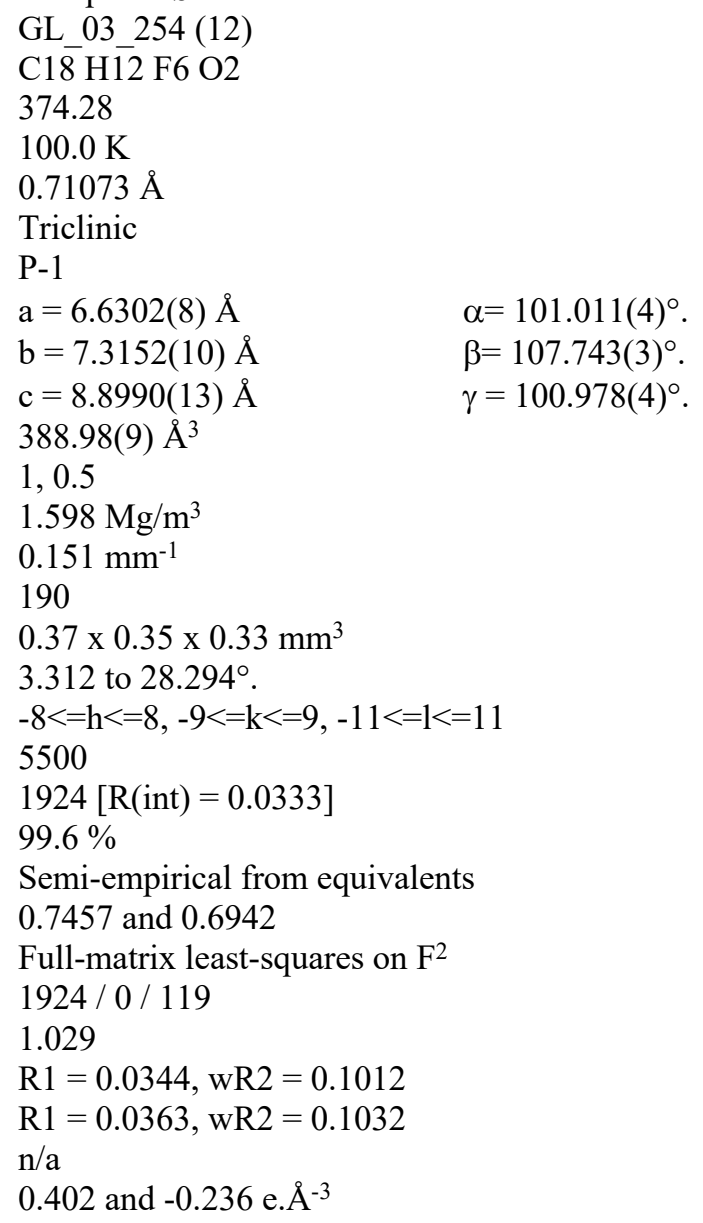


Table S3. Atomic coordinates ( $\times 10^{4}$ ) and equivalent isotropic displacement parameters $\left(\AA^{2} \times 10^{3}\right)$ for complex $\mathbf{2 b} \cdot \mathbf{4}$. $U(\mathrm{eq})$ is defined as one third of the trace of the orthogonalized $U^{\mathrm{ij}}$ tensor.

\begin{tabular}{lcccc}
\hline & $\mathrm{x}$ & $\mathrm{y}$ & $\mathrm{z}$ & $\mathrm{U}(\mathrm{eq})$ \\
\hline $\mathrm{F}(2)$ & $5130(1)$ & $2440(1)$ & $1852(1)$ & $26(1)$ \\
$\mathrm{F}(3)$ & $6587(1)$ & $6303(1)$ & $3321(1)$ & $26(1)$ \\
$\mathrm{F}(1)$ & $3544(1)$ & $1141(1)$ & $-1467(1)$ & $29(1)$ \\
$\mathrm{O}(1)$ & $8187(1)$ & $1837(1)$ & $6893(1)$ & $25(1)$ \\
$\mathrm{C}(5)$ & $9600(2)$ & $4036(1)$ & $9499(1)$ & $14(1)$ \\
$\mathrm{C}(1)$ & $10456(2)$ & $7539(1)$ & $9779(1)$ & $17(1)$ \\
$\mathrm{C}(8)$ & $5069(2)$ & $3696(2)$ & $944(1)$ & $17(1)$ \\
$\mathrm{C}(9)$ & $4263(2)$ & $3034(1)$ & $-745(1)$ & $18(1)$ \\
$\mathrm{C}(4)$ & $8873(2)$ & $3736(1)$ & $7762(1)$ & $16(1)$ \\
$\mathrm{C}(2)$ & $9703(2)$ & $7182(2)$ & $8107(1)$ & $19(1)$ \\
$\mathrm{C}(3)$ & $8902(2)$ & $5276(2)$ & $7082(1)$ & $19(1)$ \\
$\mathrm{C}(7)$ & $5805(2)$ & $5659(2)$ & $1685(1)$ & $17(1)$ \\
$\mathrm{C}(6)$ & $7533(2)$ & $1440(2)$ & $5155(1)$ & $36(1)$ \\
& & & & \\
\hline
\end{tabular}


Table S4. Bond lengths $[\AA]$ and angles $\left[{ }^{\circ}\right]$ for complex $\mathbf{2 b} \cdot \mathbf{4}$.

\begin{tabular}{|c|c|}
\hline $\mathrm{F}(2)-\mathrm{C}(8)$ & $1.3334(11)$ \\
\hline $\mathrm{F}(3)-\mathrm{C}(7)$ & $1.3398(11)$ \\
\hline $\mathrm{F}(1)-\mathrm{C}(9)$ & $1.3342(12)$ \\
\hline $\mathrm{O}(1)-\mathrm{C}(4)$ & $1.3670(12)$ \\
\hline $\mathrm{O}(1)-\mathrm{C}(6)$ & $1.4266(13)$ \\
\hline$C(5)-C(5) \# 1$ & $1.4190(18)$ \\
\hline $\mathrm{C}(5)-\mathrm{C}(1) \# 1$ & $1.4221(13)$ \\
\hline $\mathrm{C}(5)-\mathrm{C}(4)$ & $1.4297(13)$ \\
\hline $\mathrm{C}(1)-\mathrm{H}(1)$ & 0.9500 \\
\hline $\mathrm{C}(1)-\mathrm{C}(2)$ & $1.3710(14)$ \\
\hline $\mathrm{C}(8)-\mathrm{C}(9)$ & $1.3828(14)$ \\
\hline $\mathrm{C}(8)-\mathrm{C}(7)$ & $1.3817(14)$ \\
\hline $\mathrm{C}(9)-\mathrm{C}(7) \# 2$ & $1.3839(14)$ \\
\hline$C(4)-C(3)$ & $1.3772(14)$ \\
\hline $\mathrm{C}(2)-\mathrm{H}(2)$ & 0.9500 \\
\hline$C(2)-C(3)$ & $1.4124(15)$ \\
\hline $\mathrm{C}(3)-\mathrm{H}(3)$ & 0.9500 \\
\hline $\mathrm{C}(6)-\mathrm{H}(6 \mathrm{~A})$ & 0.9800 \\
\hline $\mathrm{C}(6)-\mathrm{H}(6 \mathrm{~B})$ & 0.9800 \\
\hline $\mathrm{C}(6)-\mathrm{H}(6 \mathrm{C})$ & 0.9800 \\
\hline$C(4)-O(1)-C(6)$ & $117.08(9)$ \\
\hline $\mathrm{C}(5) \# 1-\mathrm{C}(5)-\mathrm{C}(1) \# 1$ & $120.20(11)$ \\
\hline $\mathrm{C}(5) \# 1-\mathrm{C}(5)-\mathrm{C}(4)$ & $118.17(10)$ \\
\hline C(1)\#1-C(5)-C(4) & $121.62(9)$ \\
\hline $\mathrm{C}(5) \# 1-\mathrm{C}(1)-\mathrm{H}(1)$ & 120.2 \\
\hline $\mathrm{C}(2)-\mathrm{C}(1)-\mathrm{C}(5) \# 1$ & $119.59(9)$ \\
\hline $\mathrm{C}(2)-\mathrm{C}(1)-\mathrm{H}(1)$ & 120.2 \\
\hline $\mathrm{F}(2)-\mathrm{C}(8)-\mathrm{C}(9)$ & 119.94(9) \\
\hline $\mathrm{F}(2)-\mathrm{C}(8)-\mathrm{C}(7)$ & $120.15(9)$ \\
\hline $\mathrm{C}(7)-\mathrm{C}(8)-\mathrm{C}(9)$ & 119.91(9) \\
\hline $\mathrm{F}(1)-\mathrm{C}(9)-\mathrm{C}(8)$ & $120.13(9)$ \\
\hline $\mathrm{F}(1)-\mathrm{C}(9)-\mathrm{C}(7) \# 2$ & $120.07(9)$ \\
\hline $\mathrm{C}(8)-\mathrm{C}(9)-\mathrm{C}(7) \# 2$ & 119.81(9) \\
\hline
\end{tabular}




$\begin{array}{ll}\mathrm{O}(1)-\mathrm{C}(4)-\mathrm{C}(5) & 114.26(8) \\ \mathrm{O}(1)-\mathrm{C}(4)-\mathrm{C}(3) & 124.84(9) \\ \mathrm{C}(3)-\mathrm{C}(4)-\mathrm{C}(5) & 120.90(9) \\ \mathrm{C}(1)-\mathrm{C}(2)-\mathrm{H}(2) & 119.3 \\ \mathrm{C}(1)-\mathrm{C}(2)-\mathrm{C}(3) & 121.33(9) \\ \mathrm{C}(3)-\mathrm{C}(2)-\mathrm{H}(2) & 119.3 \\ \mathrm{C}(4)-\mathrm{C}(3)-\mathrm{C}(2) & 119.79(9) \\ \mathrm{C}(4)-\mathrm{C}(3)-\mathrm{H}(3) & 120.1 \\ \mathrm{C}(2)-\mathrm{C}(3)-\mathrm{H}(3) & 120.1 \\ \mathrm{~F}(3)-\mathrm{C}(7)-\mathrm{C}(8) & 120.00(9) \\ \mathrm{F}(3)-\mathrm{C}(7)-\mathrm{C}(9) \# 2 & 119.72(9) \\ \mathrm{C}(8)-\mathrm{C}(7)-\mathrm{C}(9) \# 2 & 120.28(9) \\ \mathrm{O}(1)-\mathrm{C}(6)-\mathrm{H}(6 \mathrm{~A}) & 109.5 \\ \mathrm{O}(1)-\mathrm{C}(6)-\mathrm{H}(6 \mathrm{~B}) & 109.5 \\ \mathrm{O}(1)-\mathrm{C}(6)-\mathrm{H}(6 \mathrm{C}) & 109.5 \\ \mathrm{H}(6 \mathrm{~A})-\mathrm{C}(6)-\mathrm{H}(6 \mathrm{~B}) & 109.5 \\ \mathrm{H}(6 \mathrm{~A})-\mathrm{C}(6)-\mathrm{H}(6 \mathrm{C}) & 109.5 \\ \mathrm{H}(6 \mathrm{~B})-\mathrm{C}(6)-\mathrm{H}(6 \mathrm{C}) & 109.5 \\ & \end{array}$

Symmetry transformations used to generate equivalent atoms:

$\# 1-x+2,-y+1,-z+2 \quad \# 2-x+1,-y+1,-z$ 
Table S5. Anisotropic displacement parameters $\left(\AA^{2} \times 10^{3}\right)$ for complex $\mathbf{2 b} \cdot \mathbf{4}$. The anisotropic displacement factor exponent takes the form: $-2 \pi^{2}\left[h^{2} a^{* 2} U^{11}+\ldots+2 h k a^{*} b^{*} U^{12}\right]$

\begin{tabular}{lcccccc}
\hline & $\mathrm{U}^{11}$ & $\mathrm{U}^{22}$ & $\mathrm{U}^{33}$ & $\mathrm{U}^{23}$ & $\mathrm{U}^{13}$ & $\mathrm{U}^{12}$ \\
\hline $\mathrm{F}(2)$ & $30(1)$ & $29(1)$ & $30(1)$ & $21(1)$ & $14(1)$ & $14(1)$ \\
$\mathrm{F}(3)$ & $28(1)$ & $35(1)$ & $13(1)$ & $3(1)$ & $4(1)$ & $10(1)$ \\
$\mathrm{F}(1)$ & $34(1)$ & $15(1)$ & $32(1)$ & $2(1)$ & $10(1)$ & $4(1)$ \\
$\mathrm{O}(1)$ & $34(1)$ & $20(1)$ & $14(1)$ & $0(1)$ & $8(1)$ & $1(1)$ \\
$\mathrm{C}(5)$ & $13(1)$ & $16(1)$ & $14(1)$ & $6(1)$ & $6(1)$ & $5(1)$ \\
$\mathrm{C}(1)$ & $19(1)$ & $15(1)$ & $19(1)$ & $7(1)$ & $9(1)$ & $6(1)$ \\
$\mathrm{C}(8)$ & $17(1)$ & $20(1)$ & $21(1)$ & $12(1)$ & $8(1)$ & $9(1)$ \\
$\mathrm{C}(9)$ & $17(1)$ & $15(1)$ & $21(1)$ & $4(1)$ & $7(1)$ & $5(1)$ \\
$\mathrm{C}(4)$ & $16(1)$ & $19(1)$ & $14(1)$ & $4(1)$ & $6(1)$ & $3(1)$ \\
$\mathrm{C}(2)$ & $20(1)$ & $22(1)$ & $21(1)$ & $13(1)$ & $10(1)$ & $9(1)$ \\
$\mathrm{C}(3)$ & $18(1)$ & $27(1)$ & $13(1)$ & $9(1)$ & $6(1)$ & $7(1)$ \\
$\mathrm{C}(7)$ & $16(1)$ & $23(1)$ & $14(1)$ & $5(1)$ & $5(1)$ & $7(1)$ \\
$\mathrm{C}(6)$ & $47(1)$ & $35(1)$ & $15(1)$ & $-2(1)$ & $10(1)$ & $-6(1)$ \\
\hline
\end{tabular}

Table S6. Hydrogen coordinates ( $\left.\mathrm{x} 10^{4}\right)$ and isotropic displacement parameters $\left(\AA^{2} \times 10^{3}\right)$ for sletten21R_0m_a.

\begin{tabular}{lrrrr}
\hline & $x$ & $y$ & $z$ & $U(e q)$ \\
\hline$H(1)$ & 11013 & 8830 & 10450 & 20 \\
$H(2)$ & 9719 & 8237 & 7629 & 22 \\
$H(3)$ & 8386 & 5058 & 5926 & 23 \\
H(6A) & 7153 & 43 & 4676 & 54 \\
H(6B) & 8745 & 2080 & 4865 & 54 \\
H(6C) & 6252 & 1925 & 4730 & 54 \\
& & & & \\
\hline
\end{tabular}




\subsection{Crystal data and structure for complex 3b·4 (CCDC number: 2060322)}

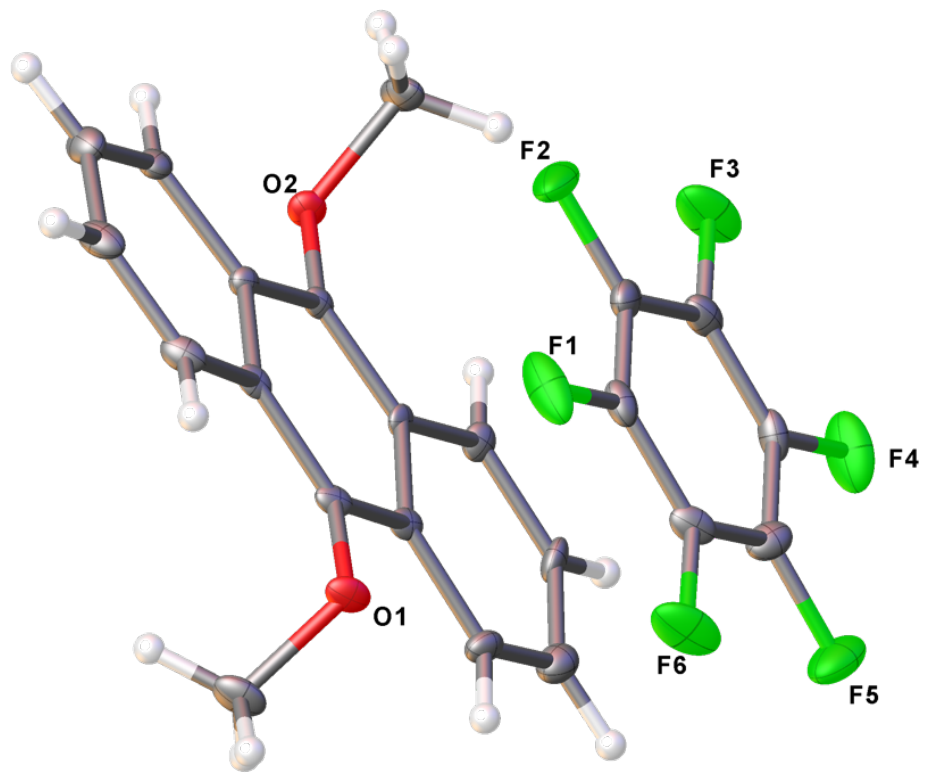

Table S7. Crystal data and structure refinement for complex $\mathbf{3 b} \cdot \mathbf{4}$.

Identification code

Empirical formula

Formula weight

Temperature

Wavelength

Crystal system

Space group

Unit cell dimensions

Volume

Z

Density (calculated)

Absorption coefficient

$\mathrm{F}(000)$

Crystal size

Theta range for data collection

Index ranges

Reflections collected

Independent reflections

Completeness to theta $=24.998^{\circ}$

Absorption correction

Max. and min. transmission

Refinement method

Data / restraints / parameters

Goodness-of-fit on $\mathrm{F}^{2}$

Final $\mathrm{R}$ indices [I $>2$ sigma(I)]

$\mathrm{R}$ indices (all data)

Extinction coefficient

Largest diff. peak and hole
GL_03_249(2)

C22 $\mathrm{H} 14$ F6 O2

424.33

$100.0 \mathrm{~K}$

$0.71073 \AA$

Monoclinic

P 21/n

$\mathrm{a}=6.903(6) \AA$

$\alpha=90^{\circ}$.

$\mathrm{b}=21.556(16) \AA$

$\beta=93.48(2)^{\circ}$.

$\mathrm{c}=11.993(9) \AA$

1781(2) $\AA^{3}$

$\gamma=90^{\circ}$.

4

$1.582 \mathrm{Mg} / \mathrm{m}^{3}$

$0.143 \mathrm{~mm}^{-1}$

864

$0.3 \times 0.14 \times 0.1 \mathrm{~mm}^{3}$

0.945 to $24.998^{\circ}$.

$-7<=\mathrm{h}<=8,-23<=\mathrm{k}<=25,-14<=\mathrm{l}<=14$

18499

$3141[\mathrm{R}(\mathrm{int})=0.0662]$

$100.0 \%$

Semi-empirical from equivalents

0.4288 and 0.2990

Full-matrix least-squares on $\mathrm{F}^{2}$

$3141 / 0 / 274$

1.093

$\mathrm{R} 1=0.1003, \mathrm{wR} 2=0.2923$

$\mathrm{R} 1=0.1211, \mathrm{wR} 2=0.3104$

$\mathrm{n} / \mathrm{a}$

1.021 and -0.781 e. $\AA^{-3}$ 
Table S8. Atomic coordinates ( $\left.\times 10^{4}\right)$ and equivalent isotropic displacement parameters $\left(\AA^{2} \times 10^{3}\right)$ for complex $\mathbf{3 b} \cdot \mathbf{4}$. $U(\mathrm{eq})$ is defined as one third of the trace of the orthogonalized $U^{\mathrm{ij}}$ tensor.

\begin{tabular}{|c|c|c|c|c|}
\hline & $\mathrm{x}$ & $\mathrm{y}$ & $\mathrm{z}$ & $\mathrm{U}(\mathrm{eq})$ \\
\hline $\mathrm{F}(2)$ & $5969(5)$ & $3033(2)$ & $3556(3)$ & $33(1)$ \\
\hline $\mathrm{F}(5)$ & $6641(5)$ & $5238(2)$ & $1426(3)$ & $34(1)$ \\
\hline $\mathrm{F}(1)$ & $6584(5)$ & $3059(2)$ & 1344(3) & $32(1)$ \\
\hline $\mathrm{O}(2)$ & $116(5)$ & $3447(2)$ & $4758(3)$ & $13(1)$ \\
\hline $\mathrm{F}(6)$ & $6869(5)$ & $4165(2)$ & $275(3)$ & $33(1)$ \\
\hline $\mathrm{F}(3)$ & $5758(5)$ & 4114(2) & 4705(3) & $36(1)$ \\
\hline $\mathrm{O}(1)$ & $2426(5)$ & $3461(2)$ & $396(3)$ & $14(1)$ \\
\hline $\mathrm{F}(4)$ & $6112(5)$ & $5213(2)$ & $3640(3)$ & $36(1)$ \\
\hline C (8) & 1782(7) & $3458(3)$ & $1474(4)$ & $12(1)$ \\
\hline$C(22)$ & $6622(7)$ & $4150(3)$ & 1374(4) & $16(1)$ \\
\hline C(9) & $1501(7)$ & $2889(2)$ & 1990(4) & 11(1) \\
\hline$C(14)$ & $897(7)$ & $2887(2)$ & $3125(4)$ & $10(1)$ \\
\hline$C(7)$ & $1562(7)$ & $4020(2)$ & 2019(4) & 11(1) \\
\hline$C(2)$ & 1051(7) & 4018(2) & $3169(4)$ & $10(1)$ \\
\hline$C(1)$ & 724(7) & $3444(2)$ & $3679(4)$ & 11(1) \\
\hline$C(18)$ & $6150(8)$ & $3576(3)$ & $3028(5)$ & $18(1)$ \\
\hline C(4) & 1161(8) & $5145(3)$ & $3213(5)$ & $15(1)$ \\
\hline$C(3)$ & $905(7)$ & $4589(2)$ & $3737(5)$ & 13(1) \\
\hline$C(17)$ & $6458(8)$ & $3586(3)$ & $1910(5)$ & $16(1)$ \\
\hline$C(19)$ & $6035(8)$ & $4126(3)$ & $3614(5)$ & $18(1)$ \\
\hline$C(20)$ & $6201(8)$ & $4678(3)$ & $3083(5)$ & $17(1)$ \\
\hline$C(15)$ & 1679(9) & $3373(3)$ & $5608(4)$ & $18(1)$ \\
\hline$C(13)$ & $484(7)$ & $2302(2)$ & $3632(5)$ & $13(1)$ \\
\hline$C(16)$ & $890(8)$ & $3423(3)$ & $-465(4)$ & $20(1)$ \\
\hline$C(6)$ & 1783(8) & $4608(3)$ & 1491(5) & $15(1)$ \\
\hline$C(21)$ & $6511(8)$ & $4690(3)$ & 1952(5) & $19(1)$ \\
\hline$C(12)$ & $708(8)$ & $1765(3)$ & $3070(5)$ & 17(1) \\
\hline$C(5)$ & $1623(8)$ & $5149(3)$ & 2066(5) & $20(1)$ \\
\hline$C(10)$ & 1781(8) & 2308(3) & $1454(5)$ & $15(1)$ \\
\hline$C(11)$ & 1391(8) & $1762(3)$ & 1977(5) & $17(1)$ \\
\hline
\end{tabular}


Table S9. Bond lengths $[\AA]$ and angles $\left[{ }^{\circ}\right]$ for complex $\mathbf{3 b} \cdot \mathbf{4}$.

\begin{tabular}{|c|c|}
\hline $\mathrm{F}(2)-\mathrm{C}(18)$ & $1.339(7)$ \\
\hline $\mathrm{F}(5)-\mathrm{C}(21)$ & $1.344(7)$ \\
\hline $\mathrm{F}(1)-\mathrm{C}(17)$ & $1.329(6)$ \\
\hline $\mathrm{O}(2)-\mathrm{C}(1)$ & $1.384(6)$ \\
\hline $\mathrm{O}(2)-\mathrm{C}(15)$ & $1.447(7)$ \\
\hline$F(6)-C(22)$ & $1.339(6)$ \\
\hline $\mathrm{F}(3)-\mathrm{C}(19)$ & $1.334(7)$ \\
\hline $\mathrm{O}(1)-\mathrm{C}(8)$ & $1.392(6)$ \\
\hline $\mathrm{O}(1)-\mathrm{C}(16)$ & $1.437(7)$ \\
\hline $\mathrm{F}(4)-\mathrm{C}(20)$ & $1.335(6)$ \\
\hline $\mathrm{C}(8)-\mathrm{C}(9)$ & $1.393(8)$ \\
\hline $\mathrm{C}(8)-\mathrm{C}(7)$ & $1.389(8)$ \\
\hline $\mathrm{C}(22)-\mathrm{C}(17)$ & $1.384(8)$ \\
\hline$C(22)-C(21)$ & $1.360(8)$ \\
\hline$C(9)-C(14)$ & $1.447(7)$ \\
\hline$C(9)-C(10)$ & $1.426(8)$ \\
\hline$C(14)-C(1)$ & $1.382(7)$ \\
\hline$C(14)-C(13)$ & $1.436(7)$ \\
\hline$C(7)-C(2)$ & $1.445(8)$ \\
\hline$C(7)-C(6)$ & $1.428(7)$ \\
\hline $\mathrm{C}(2)-\mathrm{C}(1)$ & $1.404(7)$ \\
\hline$C(2)-C(3)$ & $1.412(8)$ \\
\hline $\mathrm{C}(18)-\mathrm{C}(17)$ & $1.371(9)$ \\
\hline $\mathrm{C}(18)-\mathrm{C}(19)$ & $1.384(9)$ \\
\hline $\mathrm{C}(4)-\mathrm{H}(4)$ & 0.9500 \\
\hline$C(4)-C(3)$ & $1.369(8)$ \\
\hline$C(4)-C(5)$ & $1.431(8)$ \\
\hline $\mathrm{C}(3)-\mathrm{H}(3)$ & 0.9500 \\
\hline$C(19)-C(20)$ & $1.357(9)$ \\
\hline$C(20)-C(21)$ & $1.386(9)$ \\
\hline $\mathrm{C}(15)-\mathrm{H}(15 \mathrm{~A})$ & 0.9800 \\
\hline $\mathrm{C}(15)-\mathrm{H}(15 \mathrm{~B})$ & 0.9800 \\
\hline $\mathrm{C}(15)-\mathrm{H}(15 \mathrm{C})$ & 0.9800 \\
\hline $\mathrm{C}(13)-\mathrm{H}(13)$ & 0.9500 \\
\hline
\end{tabular}




\begin{tabular}{|c|c|}
\hline$C(13)-C(12)$ & $1.352(8)$ \\
\hline $\mathrm{C}(16)-\mathrm{H}(16 \mathrm{~A})$ & 0.9800 \\
\hline $\mathrm{C}(16)-\mathrm{H}(16 \mathrm{~B})$ & 0.9800 \\
\hline $\mathrm{C}(16)-\mathrm{H}(16 \mathrm{C})$ & 0.9800 \\
\hline $\mathrm{C}(6)-\mathrm{H}(6)$ & 0.9500 \\
\hline$C(6)-C(5)$ & $1.362(8)$ \\
\hline $\mathrm{C}(12)-\mathrm{H}(12)$ & 0.9500 \\
\hline $\mathrm{C}(12)-\mathrm{C}(11)$ & $1.420(9)$ \\
\hline $\mathrm{C}(5)-\mathrm{H}(5)$ & 0.9500 \\
\hline $\mathrm{C}(10)-\mathrm{H}(10)$ & 0.9500 \\
\hline $\mathrm{C}(10)-\mathrm{C}(11)$ & $1.367(8)$ \\
\hline $\mathrm{C}(11)-\mathrm{H}(11)$ & 0.9500 \\
\hline $\mathrm{C}(1)-\mathrm{O}(2)-\mathrm{C}(15)$ & $113.7(4)$ \\
\hline $\mathrm{C}(8)-\mathrm{O}(1)-\mathrm{C}(16)$ & $113.8(4)$ \\
\hline $\mathrm{O}(1)-\mathrm{C}(8)-\mathrm{C}(9)$ & $118.5(5)$ \\
\hline $\mathrm{C}(7)-\mathrm{C}(8)-\mathrm{O}(1)$ & $118.8(5)$ \\
\hline $\mathrm{C}(7)-\mathrm{C}(8)-\mathrm{C}(9)$ & $122.5(5)$ \\
\hline $\mathrm{F}(6)-\mathrm{C}(22)-\mathrm{C}(17)$ & $119.8(5)$ \\
\hline $\mathrm{F}(6)-\mathrm{C}(22)-\mathrm{C}(21)$ & $119.7(5)$ \\
\hline $\mathrm{C}(21)-\mathrm{C}(22)-\mathrm{C}(17)$ & $120.5(5)$ \\
\hline C(8)-C(9)-C(14) & $118.4(5)$ \\
\hline C(8)-C(9)-C(10) & $123.2(5)$ \\
\hline$C(10)-C(9)-C(14)$ & $118.4(5)$ \\
\hline $\mathrm{C}(1)-\mathrm{C}(14)-\mathrm{C}(9)$ & $119.3(5)$ \\
\hline C(1)-C(14)-C(13) & $122.3(5)$ \\
\hline $\mathrm{C}(13)-\mathrm{C}(14)-\mathrm{C}(9)$ & $118.5(5)$ \\
\hline $\mathrm{C}(8)-\mathrm{C}(7)-\mathrm{C}(2)$ & $119.0(5)$ \\
\hline$C(8)-C(7)-C(6)$ & $123.2(5)$ \\
\hline$C(6)-C(7)-C(2)$ & $117.7(5)$ \\
\hline $\mathrm{C}(1)-\mathrm{C}(2)-\mathrm{C}(7)$ & $118.3(5)$ \\
\hline$C(1)-C(2)-C(3)$ & $122.6(5)$ \\
\hline $\mathrm{C}(3)-\mathrm{C}(2)-\mathrm{C}(7)$ & $119.0(5)$ \\
\hline $\mathrm{O}(2)-\mathrm{C}(1)-\mathrm{C}(2)$ & $118.0(5)$ \\
\hline $\mathrm{C}(14)-\mathrm{C}(1)-\mathrm{O}(2)$ & $119.6(5)$ \\
\hline $\mathrm{C}(14)-\mathrm{C}(1)-\mathrm{C}(2)$ & $122.4(5)$ \\
\hline
\end{tabular}




\begin{tabular}{|c|c|}
\hline $\mathrm{F}(2)-\mathrm{C}(18)-\mathrm{C}(17)$ & $120.0(5)$ \\
\hline $\mathrm{F}(2)-\mathrm{C}(18)-\mathrm{C}(19)$ & $120.0(5)$ \\
\hline$C(17)-C(18)-C(19)$ & $120.0(5)$ \\
\hline $\mathrm{C}(3)-\mathrm{C}(4)-\mathrm{H}(4)$ & 120.4 \\
\hline$C(3)-C(4)-C(5)$ & $119.3(5)$ \\
\hline $\mathrm{C}(5)-\mathrm{C}(4)-\mathrm{H}(4)$ & 120.4 \\
\hline $\mathrm{C}(2)-\mathrm{C}(3)-\mathrm{H}(3)$ & 119.1 \\
\hline $\mathrm{C}(4)-\mathrm{C}(3)-\mathrm{C}(2)$ & $121.8(5)$ \\
\hline $\mathrm{C}(4)-\mathrm{C}(3)-\mathrm{H}(3)$ & 119.1 \\
\hline $\mathrm{F}(1)-\mathrm{C}(17)-\mathrm{C}(22)$ & $120.3(5)$ \\
\hline $\mathrm{F}(1)-\mathrm{C}(17)-\mathrm{C}(18)$ & $120.4(5)$ \\
\hline$C(18)-C(17)-C(22)$ & $119.3(5)$ \\
\hline $\mathrm{F}(3)-\mathrm{C}(19)-\mathrm{C}(18)$ & $119.8(5)$ \\
\hline $\mathrm{F}(3)-\mathrm{C}(19)-\mathrm{C}(20)$ & $119.9(5)$ \\
\hline$C(20)-C(19)-C(18)$ & $120.3(5)$ \\
\hline $\mathrm{F}(4)-\mathrm{C}(20)-\mathrm{C}(19)$ & $121.0(5)$ \\
\hline $\mathrm{F}(4)-\mathrm{C}(20)-\mathrm{C}(21)$ & $119.2(5)$ \\
\hline$C(19)-C(20)-C(21)$ & $119.8(5)$ \\
\hline $\mathrm{O}(2)-\mathrm{C}(15)-\mathrm{H}(15 \mathrm{~A})$ & 109.5 \\
\hline $\mathrm{O}(2)-\mathrm{C}(15)-\mathrm{H}(15 \mathrm{~B})$ & 109.5 \\
\hline $\mathrm{O}(2)-\mathrm{C}(15)-\mathrm{H}(15 \mathrm{C})$ & 109.5 \\
\hline $\mathrm{H}(15 \mathrm{~A})-\mathrm{C}(15)-\mathrm{H}(15 \mathrm{~B})$ & 109.5 \\
\hline $\mathrm{H}(15 \mathrm{~A})-\mathrm{C}(15)-\mathrm{H}(15 \mathrm{C})$ & 109.5 \\
\hline $\mathrm{H}(15 \mathrm{~B})-\mathrm{C}(15)-\mathrm{H}(15 \mathrm{C})$ & 109.5 \\
\hline $\mathrm{C}(14)-\mathrm{C}(13)-\mathrm{H}(13)$ & 119.7 \\
\hline$C(12)-C(13)-C(14)$ & $120.6(5)$ \\
\hline $\mathrm{C}(12)-\mathrm{C}(13)-\mathrm{H}(13)$ & 119.7 \\
\hline $\mathrm{O}(1)-\mathrm{C}(16)-\mathrm{H}(16 \mathrm{~A})$ & 109.5 \\
\hline $\mathrm{O}(1)-\mathrm{C}(16)-\mathrm{H}(16 \mathrm{~B})$ & 109.5 \\
\hline $\mathrm{O}(1)-\mathrm{C}(16)-\mathrm{H}(16 \mathrm{C})$ & 109.5 \\
\hline $\mathrm{H}(16 \mathrm{~A})-\mathrm{C}(16)-\mathrm{H}(16 \mathrm{~B})$ & 109.5 \\
\hline$H(16 A)-C(16)-H(16 C)$ & 109.5 \\
\hline $\mathrm{H}(16 \mathrm{~B})-\mathrm{C}(16)-\mathrm{H}(16 \mathrm{C})$ & 109.5 \\
\hline$C(7)-C(6)-H(6)$ & 119.3 \\
\hline$C(5)-C(6)-C(7)$ & $121.4(5)$ \\
\hline $\mathrm{C}(5)-\mathrm{C}(6)-\mathrm{H}(6)$ & 119.3 \\
\hline
\end{tabular}




$\begin{array}{ll}\mathrm{F}(5)-\mathrm{C}(21)-\mathrm{C}(22) & 120.4(5) \\ \mathrm{F}(5)-\mathrm{C}(21)-\mathrm{C}(20) & 119.6(5) \\ \mathrm{C}(22)-\mathrm{C}(21)-\mathrm{C}(20) & 120.0(5) \\ \mathrm{C}(13)-\mathrm{C}(12)-\mathrm{H}(12) & 119.4 \\ \mathrm{C}(13)-\mathrm{C}(12)-\mathrm{C}(11) & 121.2(5) \\ \mathrm{C}(11)-\mathrm{C}(12)-\mathrm{H}(12) & 119.4 \\ \mathrm{C}(4)-\mathrm{C}(5)-\mathrm{H}(5) & 119.6 \\ \mathrm{C}(6)-\mathrm{C}(5)-\mathrm{C}(4) & 120.8(5) \\ \mathrm{C}(6)-\mathrm{C}(5)-\mathrm{H}(5) & 119.6 \\ \mathrm{C}(9)-\mathrm{C}(10)-\mathrm{H}(10) & 119.6 \\ \mathrm{C}(11)-\mathrm{C}(10)-\mathrm{C}(9) & 120.8(5) \\ \mathrm{C}(11)-\mathrm{C}(10)-\mathrm{H}(10) & 119.6 \\ \mathrm{C}(12)-\mathrm{C}(11)-\mathrm{H}(11) & 119.8 \\ \mathrm{C}(10)-\mathrm{C}(11)-\mathrm{C}(12) & 120.4(5) \\ \mathrm{C}(10)-\mathrm{C}(11)-\mathrm{H}(11) & 119.8\end{array}$

Symmetry transformations used to generate equivalent atoms: 
Table S10. Anisotropic displacement parameters $\left(\AA^{2} \times 10^{3}\right)$ for complex $\mathbf{3 b} \cdot \mathbf{4}$. The anisotropic displacement factor exponent takes the form: $-2 \pi^{2}\left[h^{2} a^{* 2} U^{11}+\ldots+2 h k a^{*} b^{*} U^{12}\right]$

\begin{tabular}{|c|c|c|c|c|c|c|}
\hline & $\mathrm{U}^{11}$ & $\mathrm{U}^{22}$ & $\mathrm{U}^{33}$ & $\mathrm{U}^{23}$ & $\mathrm{U}^{13}$ & $\mathrm{U}^{12}$ \\
\hline $\mathrm{F}(2)$ & $23(2)$ & $25(2)$ & $50(2)$ & $25(2)$ & $-8(2)$ & $-5(2)$ \\
\hline $\mathrm{F}(5)$ & $26(2)$ & $23(2)$ & $52(3)$ & $18(2)$ & $5(2)$ & $4(2)$ \\
\hline $\mathrm{F}(1)$ & $23(2)$ & $24(2)$ & $48(2)$ & $-25(2)$ & $-11(2)$ & $11(2)$ \\
\hline $\mathrm{O}(2)$ & $13(2)$ & $18(2)$ & $9(2)$ & $0(2)$ & $5(1)$ & $0(2)$ \\
\hline $\mathrm{F}(6)$ & $27(2)$ & $63(3)$ & $10(2)$ & $1(2)$ & $7(1)$ & $9(2)$ \\
\hline $\mathrm{F}(3)$ & $25(2)$ & $72(3)$ & $10(2)$ & $-1(2)$ & $7(1)$ & $1(2)$ \\
\hline $\mathrm{O}(1)$ & $12(2)$ & $24(2)$ & $8(2)$ & $1(2)$ & $5(1)$ & $3(2)$ \\
\hline $\mathrm{F}(4)$ & $26(2)$ & $31(2)$ & $51(3)$ & $-29(2)$ & $2(2)$ & $3(2)$ \\
\hline $\mathrm{C}(8)$ & $5(2)$ & 19(3) & $12(3)$ & $3(2)$ & $1(2)$ & $3(2)$ \\
\hline$C(22)$ & $10(3)$ & $30(3)$ & $8(2)$ & $-3(2)$ & $4(2)$ & $7(2)$ \\
\hline$C(9)$ & $4(2)$ & $18(3)$ & $12(3)$ & $-2(2)$ & $1(2)$ & $3(2)$ \\
\hline$C(14)$ & $7(2)$ & $12(3)$ & 11(3) & $4(2)$ & $1(2)$ & $-2(2)$ \\
\hline$C(7)$ & $4(2)$ & $15(3)$ & $14(3)$ & $2(2)$ & $1(2)$ & $2(2)$ \\
\hline$C(2)$ & $4(2)$ & $13(3)$ & $14(3)$ & $3(2)$ & $2(2)$ & $-3(2)$ \\
\hline$C(1)$ & $5(2)$ & $17(3)$ & $10(3)$ & $-1(2)$ & $1(2)$ & $1(2)$ \\
\hline$C(18)$ & $9(3)$ & $20(3)$ & $24(3)$ & $5(2)$ & $-2(2)$ & $-5(2)$ \\
\hline$C(4)$ & 11(3) & $9(3)$ & $26(3)$ & $1(2)$ & $5(2)$ & $-6(2)$ \\
\hline$C(3)$ & $8(2)$ & $17(3)$ & $15(3)$ & $0(2)$ & $4(2)$ & $1(2)$ \\
\hline$C(17)$ & $9(3)$ & $16(3)$ & $24(3)$ & $-7(2)$ & $-3(2)$ & $1(2)$ \\
\hline$C(19)$ & $9(3)$ & $33(3)$ & $12(3)$ & $2(2)$ & $2(2)$ & $-1(2)$ \\
\hline$C(20)$ & $10(3)$ & $18(3)$ & $23(3)$ & $-13(2)$ & $1(2)$ & $-1(2)$ \\
\hline$C(15)$ & $26(3)$ & $18(3)$ & $8(3)$ & $0(2)$ & $-2(2)$ & $-2(2)$ \\
\hline$C(13)$ & $10(2)$ & $16(3)$ & $14(3)$ & $3(2)$ & $-2(2)$ & $2(2)$ \\
\hline$C(16)$ & $21(3)$ & $31(3)$ & $7(3)$ & $-2(2)$ & $1(2)$ & $7(3)$ \\
\hline$C(6)$ & $12(3)$ & $17(3)$ & $16(3)$ & $7(2)$ & $3(2)$ & $-1(2)$ \\
\hline $\mathrm{C}(21)$ & $14(3)$ & $15(3)$ & $27(3)$ & $11(2)$ & $4(2)$ & $4(2)$ \\
\hline$C(12)$ & $23(3)$ & $10(3)$ & $19(3)$ & $1(2)$ & $-4(2)$ & $-2(2)$ \\
\hline$C(5)$ & $15(3)$ & $18(3)$ & $26(3)$ & $6(2)$ & $0(2)$ & $1(2)$ \\
\hline$C(10)$ & $15(3)$ & $19(3)$ & $12(3)$ & $-2(2)$ & $1(2)$ & $6(2)$ \\
\hline $\mathrm{C}(11)$ & $19(3)$ & $14(3)$ & $18(3)$ & $-3(2)$ & $-4(2)$ & $8(2)$ \\
\hline
\end{tabular}


Table S11. Hydrogen coordinates ( $\left.\times 10^{4}\right)$ and isotropic displacement parameters $\left(\AA^{2} \times 10^{3}\right)$ for complex $3 \mathbf{b} \cdot \mathbf{4}$.

\begin{tabular}{lrrrr}
\hline & $x$ & $y$ & $z$ & $U(e q)$ \\
& & & & \\
$H(4)$ & 1033 & 5523 & 3607 & 18 \\
$H(3)$ & 621 & 4587 & 4502 & 16 \\
$\mathrm{H}(15 \mathrm{~A})$ & 2444 & 3005 & 5442 & 26 \\
$\mathrm{H}(15 \mathrm{~B})$ & 1138 & 3324 & 6339 & 26 \\
$\mathrm{H}(15 \mathrm{C})$ & 2515 & 3741 & 5618 & 26 \\
$\mathrm{H}(13)$ & 48 & 2292 & 4368 & 16 \\
$\mathrm{H}(16 \mathrm{~A})$ & 1446 & 3377 & -1192 & 30 \\
$\mathrm{H}(16 \mathrm{~B})$ & 107 & 3803 & -462 & 30 \\
$\mathrm{H}(16 \mathrm{C})$ & 67 & 3064 & -326 & 30 \\
$\mathrm{H}(6)$ & 2048 & 4622 & 723 & 18 \\
$\mathrm{H}(12)$ & 404 & 1383 & 3413 & 21 \\
$\mathrm{H}(5)$ & 1819 & 5533 & 1702 & 24 \\
$\mathrm{H}(10)$ & 2245 & 2300 & 724 & 19 \\
$\mathrm{H}(11)$ & 1578 & 1379 & 1607 & 21 \\
\hline
\end{tabular}




\subsection{Crystal data and structure for complex 1b·5a (CCDC number: 2060323)}

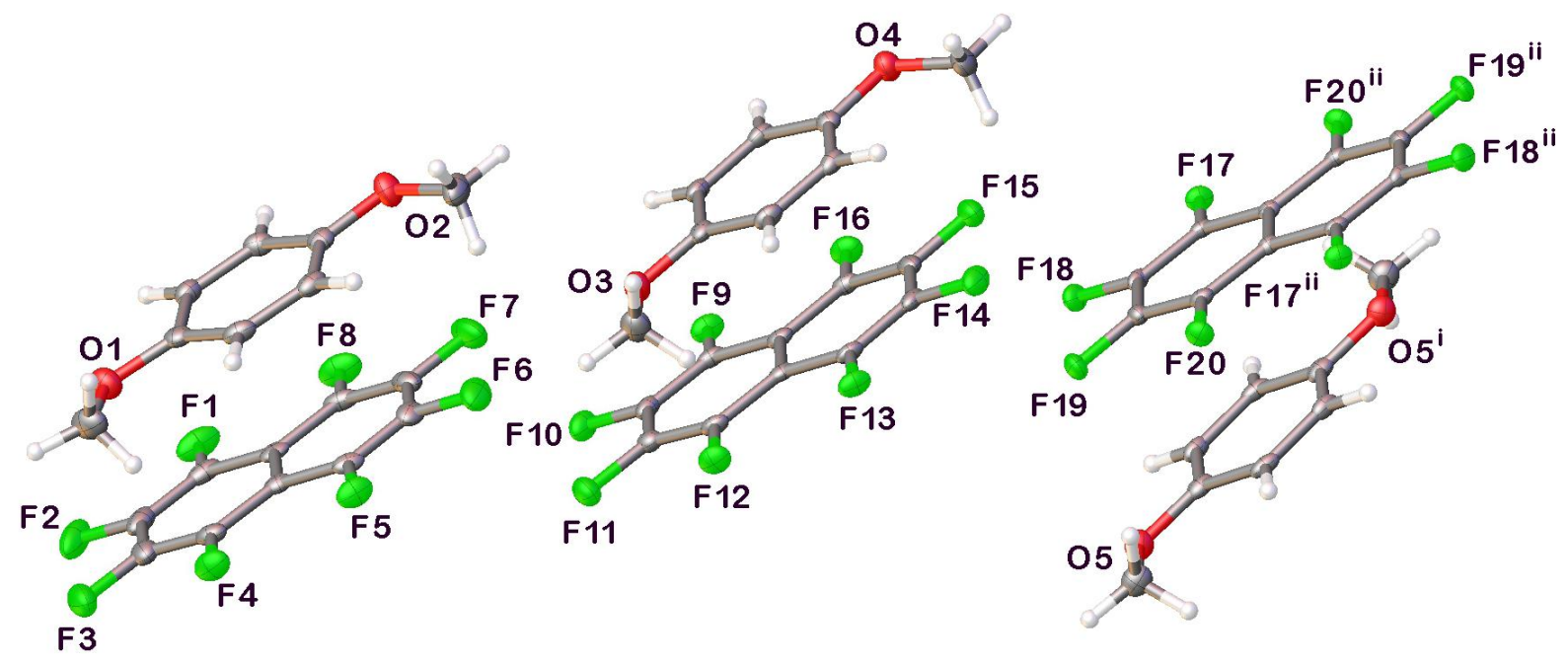

Table S12. Crystal data and structure refinement for complex $\mathbf{1 b} \cdot \mathbf{5 a}$.

Identification code

Empirical formula

Formula weight

Temperature

Wavelength

Crystal system

Space group

Unit cell dimensions

Volume

Z, Z'

Density (calculated)

Absorption coefficient

$\mathrm{F}(000)$

Crystal size

Theta range for data collection

Index ranges

Reflections collected

Independent reflections

Completeness to theta $=25.242^{\circ}$

Absorption correction

Max. and min. transmission

Refinement method

Data / restraints / parameters

Goodness-of-fit on $\mathrm{F}^{2}$

Final $\mathrm{R}$ indices [I $>2 \operatorname{sigma}(\mathrm{I})]$

$\mathrm{R}$ indices (all data)

Extinction coefficient

Largest diff. peak and hole
GL_03_249 3

$\mathrm{C} 18 \mathrm{H} 10 \mathrm{~F} 8 \mathrm{O} 2$

410.26

$100.0 \mathrm{~K}$

$0.71073 \AA$

Triclinic

P-1

$\mathrm{a}=6.253(6) \AA$

$\mathrm{b}=8.019(7) \AA$

$\mathrm{c}=40.52(4) \AA$

2024(3) $\AA^{3}$

$5,2.5$

$1.683 \mathrm{Mg} / \mathrm{m}^{3}$

$0.171 \mathrm{~mm}^{-1}$

1030

$0.28 \times 0.23 \times 0.22 \mathrm{~mm}^{3}$

2.018 to $27.081^{\circ}$.

$-7<=\mathrm{h}<=7,-10<=\mathrm{k}<=10,-51<=1<=50$

23085

$8607[\mathrm{R}(\mathrm{int})=0.0430]$

$98.7 \%$

Semi-empirical from equivalents

0.4912 and 0.4217

Full-matrix least-squares on $\mathrm{F}^{2}$

8607 / 0 / 636

1.075

$\mathrm{R} 1=0.0585, \mathrm{wR} 2=0.1405$

$\mathrm{R} 1=0.0771, \mathrm{wR} 2=0.1508$

$\mathrm{n} / \mathrm{a}$

0.422 and -0.376 e. $\AA^{-3}$ $\alpha=93.251(9)^{\circ}$.

$\beta=93.799(10)^{\circ}$.

$\gamma=90.921(9)^{\circ}$. 
Table S13. Atomic coordinates ( x 10 $)$ and equivalent isotropic displacement parameters $\left(\AA^{2} \times 10^{3}\right)$ for complex 1b·5a. $U(e q)$ is defined as one third of the trace of the orthogonalized $U^{i j}$ tensor.

\begin{tabular}{|c|c|c|c|c|}
\hline & $\mathrm{x}$ & $\mathrm{y}$ & $\mathrm{z}$ & $\mathrm{U}(\mathrm{eq})$ \\
\hline $\mathrm{F}(001)$ & $5618(2)$ & $3298(2)$ & $4960(1)$ & $17(1)$ \\
\hline $\mathrm{F}(002)$ & $13486(2)$ & $5111(2)$ & $5557(1)$ & 19(1) \\
\hline $\mathrm{F}(003)$ & $12255(2)$ & $5360(2)$ & 6951(1) & $21(1)$ \\
\hline $\mathrm{F}(004)$ & $6772(2)$ & $2402(2)$ & $5574(1)$ & $20(1)$ \\
\hline $\mathrm{F}(005)$ & $3511(2)$ & 1951(2) & $6907(1)$ & $22(1)$ \\
\hline$F(006)$ & $11421(2)$ & $3836(2)$ & $7477(1)$ & $23(1)$ \\
\hline $\mathrm{F}(007)$ & $4320(2)$ & $3511(2)$ & 6381(1) & $24(1)$ \\
\hline $\mathrm{F}(008)$ & $10687(2)$ & $3255(2)$ & $5868(1)$ & $20(1)$ \\
\hline $\mathrm{F}(009)$ & $8989(2)$ & $2946(2)$ & $9448(1)$ & $28(1)$ \\
\hline $\mathrm{F}(00 \mathrm{~A})$ & $9755(2)$ & $4335(2)$ & $8904(1)$ & $26(1)$ \\
\hline $\mathrm{F}(00 \mathrm{~B})$ & $10975(2)$ & $6289(2)$ & $6342(1)$ & $26(1)$ \\
\hline $\mathrm{F}(00 \mathrm{C})$ & $4733(2)$ & $1138(2)$ & $7526(1)$ & $25(1)$ \\
\hline $\mathrm{F}(00 \mathrm{D})$ & $7040(2)$ & $5418(2)$ & 6064(1) & $27(1)$ \\
\hline $\mathrm{F}(00 \mathrm{E})$ & $8643(2)$ & $2051(2)$ & $7807(1)$ & $26(1)$ \\
\hline $\mathrm{F}(00 \mathrm{~F})$ & $1088(2)$ & $867(2)$ & $8906(1)$ & $34(1)$ \\
\hline $\mathrm{F}(00 \mathrm{G})$ & $1840(2)$ & $2272(2)$ & $8363(1)$ & $35(1)$ \\
\hline $\mathrm{F}(00 \mathrm{H})$ & $8422(3)$ & $5104(2)$ & $8290(1)$ & $34(1)$ \\
\hline $\mathrm{F}(00 \mathrm{I})$ & 4514(3) & $4096(2)$ & $8020(1)$ & $39(1)$ \\
\hline $\mathrm{F}(00 \mathrm{~J})$ & $6315(3)$ & $1168(2)$ & 9794(1) & $39(1)$ \\
\hline $\mathrm{F}(00 \mathrm{~K})$ & 2384(3) & $152(2)$ & $9526(1)$ & $40(1)$ \\
\hline $\mathrm{O}(00 \mathrm{~L})$ & 9195(3) & $7246(2)$ & $7670(1)$ & $23(1)$ \\
\hline $\mathrm{O}(00 \mathrm{M})$ & $11249(3)$ & $-1305(2)$ & $5603(1)$ & $23(1)$ \\
\hline $\mathrm{O}(00 \mathrm{~N})$ & 6786(3) & $9517(2)$ & $6434(1)$ & $24(1)$ \\
\hline $\mathrm{O}(00 \mathrm{O})$ & $5768(3)$ & $6117(2)$ & $9688(1)$ & $28(1)$ \\
\hline $\mathrm{O}(00 \mathrm{P})$ & $4826(3)$ & $8478(2)$ & $8425(1)$ & $27(1)$ \\
\hline $\mathrm{C}(00 \mathrm{Q})$ & 8973(3) & $4763(2)$ & 4921(1) & 11(1) \\
\hline $\mathrm{C}(00 \mathrm{R})$ & 11562(3) & $4717(2)$ & $5403(1)$ & $13(1)$ \\
\hline $\mathrm{C}(00 \mathrm{~S})$ & 7559(3) & $3797(2)$ & $5099(1)$ & $12(1)$ \\
\hline $\mathrm{C}(00 \mathrm{~T})$ & 8912(3) & $3913(2)$ & 7004(1) & $14(1)$ \\
\hline $\mathrm{C}(00 \mathrm{U})$ & 10147(3) & $3780(3)$ & $5564(1)$ & $15(1)$ \\
\hline $\mathrm{C}(00 \mathrm{~V})$ & $6253(3)$ & $3945(3)$ & 6531(1) & $16(1)$ \\
\hline
\end{tabular}




\begin{tabular}{|c|c|c|c|c|}
\hline $\mathrm{C}(00 \mathrm{~W})$ & $6456(3)$ & 2877(3) & $8977(1)$ & $16(1)$ \\
\hline $\mathrm{C}(00 \mathrm{X})$ & 7057(4) & 2455(3) & $9305(1)$ & $19(1)$ \\
\hline $\mathrm{C}(00 \mathrm{Y})$ & $6059(4)$ & 2041(3) & $7353(1)$ & $18(1)$ \\
\hline $\mathrm{C}(00 \mathrm{Z})$ & $8130(3)$ & $3324(2)$ & $5411(1)$ & $14(1)$ \\
\hline$C(010)$ & 9484(3) & $3417(3)$ & $7330(1)$ & $16(1)$ \\
\hline$C(011)$ & 8091(4) & $2520(3)$ & $7499(1)$ & $18(1)$ \\
\hline $\mathrm{C}(012)$ & $6842(3)$ & $3426(3)$ & $6854(1)$ & $15(1)$ \\
\hline C(013) & $12074(3)$ & $402(3)$ & $5137(1)$ & $18(1)$ \\
\hline$C(014)$ & $5459(3)$ & 2471(3) & $7040(1)$ & $16(1)$ \\
\hline$C(015)$ & $4377(3)$ & 2335(3) & $8834(1)$ & $18(1)$ \\
\hline$C(016)$ & 10291(3) & 4876(3) & 6821(1) & $15(1)$ \\
\hline $\mathrm{C}(017)$ & $7537(4)$ & 9023(3) & $6740(1)$ & $16(1)$ \\
\hline$C(018)$ & $3765(4)$ & 2767(3) & $8506(1)$ & $22(1)$ \\
\hline $\mathrm{C}(019)$ & 9664(4) & $5367(3)$ & $6511(1)$ & $18(1)$ \\
\hline $\mathrm{C}(01 \mathrm{~A})$ & 7790(3) & 3811(3) & $8781(1)$ & $18(1)$ \\
\hline $\mathrm{C}(01 \mathrm{~B})$ & $5708(4)$ & $6769(3)$ & $9380(1)$ & $19(1)$ \\
\hline$C(01 C)$ & 10703(3) & $-604(3)$ & $5306(1)$ & $18(1)$ \\
\hline$C(01 D)$ & $7619(4)$ & $4900(3)$ & $6367(1)$ & $18(1)$ \\
\hline $\mathrm{C}(01 \mathrm{E})$ & 9599(3) & $9337(3)$ & $6880(1)$ & $17(1)$ \\
\hline $\mathrm{C}(01 \mathrm{~F})$ & $8743(4)$ & 7898(3) & $7366(1)$ & $16(1)$ \\
\hline $\mathrm{C}(01 \mathrm{G})$ & $5108(4)$ & $3681(3)$ & $8329(1)$ & $24(1)$ \\
\hline $\mathrm{C}(01 \mathrm{H})$ & 3042(4) & 1406(3) & $9031(1)$ & $23(1)$ \\
\hline $\mathrm{C}(01 \mathrm{I})$ & $5236(4)$ & 7939(3) & $8740(1)$ & 19(1) \\
\hline $\mathrm{C}(01 \mathrm{~J})$ & $8648(3)$ & $-996(3)$ & $5167(1)$ & $17(1)$ \\
\hline $\mathrm{C}(01 \mathrm{~K})$ & $7130(4)$ & 4201(3) & $8469(1)$ & $22(1)$ \\
\hline $\mathrm{C}(01 \mathrm{~L})$ & 7403(4) & 7591(3) & $9248(1)$ & $21(1)$ \\
\hline $\mathrm{C}(01 \mathrm{M})$ & $3768(4)$ & $6535(3)$ & $9189(1)$ & $20(1)$ \\
\hline $\mathrm{C}(01 \mathrm{~N})$ & $10205(3)$ & 8791(3) & 7194(1) & $18(1)$ \\
\hline $\mathrm{C}(010)$ & $6666(3)$ & $7584(3)$ & $7224(1)$ & $18(1)$ \\
\hline $\mathrm{C}(01 \mathrm{P})$ & $6058(3)$ & $8140(3)$ & $6915(1)$ & $17(1)$ \\
\hline$C(01 Q)$ & $7171(4)$ & $8167(3)$ & $8926(1)$ & $21(1)$ \\
\hline$C(01 R)$ & $5713(4)$ & 1558(3) & $9485(1)$ & $26(1)$ \\
\hline $\mathrm{C}(01 \mathrm{~S})$ & $3692(4)$ & $1035(3)$ & $9346(1)$ & $27(1)$ \\
\hline $\mathrm{C}(01 \mathrm{~T})$ & $3521(4)$ & 7106(3) & $8874(1)$ & $19(1)$ \\
\hline $\mathrm{C}(01 \mathrm{U})$ & $11259(4)$ & 7633(3) & $7832(1)$ & $28(1)$ \\
\hline $\mathrm{C}(01 \mathrm{~V})$ & $8359(4)$ & $10150(3)$ & $6227(1)$ & $27(1)$ \\
\hline
\end{tabular}




\begin{tabular}{lrlll}
$\mathrm{C}(01 \mathrm{~W})$ & $13283(4)$ & $-831(3)$ & $5768(1)$ & $25(1)$ \\
$\mathrm{C}(01 \mathrm{X})$ & $6593(4)$ & $9187(3)$ & $8269(1)$ & $31(1)$ \\
$\mathrm{C}(01 \mathrm{Y})$ & $7650(4)$ & $6465(3)$ & $9901(1)$ & $33(1)$ \\
\hline
\end{tabular}


Table S14. Bond lengths $[\AA]$ and angles $\left[{ }^{\circ}\right]$ for complex $\mathbf{1 b} \cdot \mathbf{5 a}$.

\begin{tabular}{|c|c|}
\hline $\mathrm{F}(001)-\mathrm{C}(00 \mathrm{~S})$ & $1.347(3)$ \\
\hline $\mathrm{F}(002)-\mathrm{C}(00 \mathrm{R})$ & $1.342(3)$ \\
\hline $\mathrm{F}(003)-\mathrm{C}(016)$ & $1.346(3)$ \\
\hline $\mathrm{F}(004)-\mathrm{C}(00 \mathrm{Z})$ & $1.348(2)$ \\
\hline $\mathrm{F}(005)-\mathrm{C}(014)$ & $1.348(3)$ \\
\hline $\mathrm{F}(006)-\mathrm{C}(010)$ & $1.343(3)$ \\
\hline $\mathrm{F}(007)-\mathrm{C}(00 \mathrm{~V})$ & $1.348(3)$ \\
\hline $\mathrm{F}(008)-\mathrm{C}(00 \mathrm{U})$ & $1.348(3)$ \\
\hline $\mathrm{F}(009)-\mathrm{C}(00 \mathrm{X})$ & $1.348(3)$ \\
\hline $\mathrm{F}(00 \mathrm{~A})-\mathrm{C}(01 \mathrm{~A})$ & $1.346(3)$ \\
\hline $\mathrm{F}(00 \mathrm{~B})-\mathrm{C}(019)$ & $1.342(3)$ \\
\hline $\mathrm{F}(00 \mathrm{C})-\mathrm{C}(00 \mathrm{Y})$ & $1.348(3)$ \\
\hline $\mathrm{F}(00 \mathrm{D})-\mathrm{C}(01 \mathrm{D})$ & $1.346(3)$ \\
\hline $\mathrm{F}(00 \mathrm{E})-\mathrm{C}(011)$ & $1.348(3)$ \\
\hline $\mathrm{F}(00 \mathrm{~F})-\mathrm{C}(01 \mathrm{H})$ & $1.346(3)$ \\
\hline $\mathrm{F}(00 \mathrm{G})-\mathrm{C}(018)$ & $1.345(3)$ \\
\hline $\mathrm{F}(00 \mathrm{H})-\mathrm{C}(01 \mathrm{~K})$ & $1.349(3)$ \\
\hline $\mathrm{F}(00 \mathrm{I})-\mathrm{C}(01 \mathrm{G})$ & $1.344(3)$ \\
\hline $\mathrm{F}(00 \mathrm{~J})-\mathrm{C}(01 \mathrm{R})$ & $1.339(3)$ \\
\hline $\mathrm{F}(00 \mathrm{~K})-\mathrm{C}(01 \mathrm{~S})$ & $1.350(3)$ \\
\hline $\mathrm{O}(00 \mathrm{~L})-\mathrm{C}(01 \mathrm{~F})$ & $1.377(3)$ \\
\hline $\mathrm{O}(00 \mathrm{~L})-\mathrm{C}(01 \mathrm{U})$ & $1.431(3)$ \\
\hline $\mathrm{O}(00 \mathrm{M})-\mathrm{C}(01 \mathrm{C})$ & $1.380(3)$ \\
\hline $\mathrm{O}(00 \mathrm{M})-\mathrm{C}(01 \mathrm{~W})$ & $1.433(3)$ \\
\hline $\mathrm{O}(00 \mathrm{~N})-\mathrm{C}(017)$ & $1.379(3)$ \\
\hline $\mathrm{O}(00 \mathrm{~N})-\mathrm{C}(01 \mathrm{~V})$ & $1.437(3)$ \\
\hline $\mathrm{O}(00 \mathrm{O})-\mathrm{C}(01 \mathrm{~B})$ & $1.378(3)$ \\
\hline $\mathrm{O}(00 \mathrm{O})-\mathrm{C}(01 \mathrm{Y})$ & $1.428(3)$ \\
\hline $\mathrm{O}(00 \mathrm{P})-\mathrm{C}(01 \mathrm{I})$ & $1.379(3)$ \\
\hline $\mathrm{O}(00 \mathrm{P})-\mathrm{C}(01 \mathrm{X})$ & $1.434(3)$ \\
\hline $\mathrm{C}(00 \mathrm{Q})-\mathrm{C}(00 \mathrm{Q}) \# 1$ & $1.432(4)$ \\
\hline $\mathrm{C}(00 \mathrm{Q})-\mathrm{C}(00 \mathrm{R}) \# 1$ & $1.421(3)$ \\
\hline $\mathrm{C}(00 \mathrm{Q})-\mathrm{C}(00 \mathrm{~S})$ & $1.423(3)$ \\
\hline $\mathrm{C}(00 \mathrm{R})-\mathrm{C}(00 \mathrm{U})$ & $1.372(3)$ \\
\hline
\end{tabular}




\begin{tabular}{|c|c|}
\hline $\mathrm{C}(00 \mathrm{~S})-\mathrm{C}(00 \mathrm{Z})$ & $1.367(3)$ \\
\hline $\mathrm{C}(00 \mathrm{~T})-\mathrm{C}(010)$ & $1.424(3)$ \\
\hline $\mathrm{C}(00 \mathrm{~T})-\mathrm{C}(012)$ & $1.432(3)$ \\
\hline $\mathrm{C}(00 \mathrm{~T})-\mathrm{C}(016)$ & $1.421(3)$ \\
\hline $\mathrm{C}(00 \mathrm{U})-\mathrm{C}(00 \mathrm{Z})$ & $1.401(3)$ \\
\hline $\mathrm{C}(00 \mathrm{~V})-\mathrm{C}(012)$ & $1.422(3)$ \\
\hline $\mathrm{C}(00 \mathrm{~V})-\mathrm{C}(01 \mathrm{D})$ & $1.369(3)$ \\
\hline $\mathrm{C}(00 \mathrm{~W})-\mathrm{C}(00 \mathrm{X})$ & $1.418(3)$ \\
\hline $\mathrm{C}(00 \mathrm{~W})-\mathrm{C}(015)$ & $1.438(3)$ \\
\hline $\mathrm{C}(00 \mathrm{~W})-\mathrm{C}(01 \mathrm{~A})$ & $1.420(3)$ \\
\hline $\mathrm{C}(00 \mathrm{X})-\mathrm{C}(01 \mathrm{R})$ & $1.371(4)$ \\
\hline $\mathrm{C}(00 \mathrm{Y})-\mathrm{C}(011)$ & $1.404(3)$ \\
\hline $\mathrm{C}(00 \mathrm{Y})-\mathrm{C}(014)$ & $1.365(3)$ \\
\hline $\mathrm{C}(010)-\mathrm{C}(011)$ & $1.365(3)$ \\
\hline$C(012)-C(014)$ & $1.425(3)$ \\
\hline $\mathrm{C}(013)-\mathrm{H}(013)$ & 0.9500 \\
\hline $\mathrm{C}(013)-\mathrm{C}(01 \mathrm{C})$ & $1.406(3)$ \\
\hline $\mathrm{C}(013)-\mathrm{C}(01 \mathrm{~J}) \# 2$ & $1.396(3)$ \\
\hline $\mathrm{C}(015)-\mathrm{C}(018)$ & $1.421(3)$ \\
\hline $\mathrm{C}(015)-\mathrm{C}(01 \mathrm{H})$ & $1.423(3)$ \\
\hline $\mathrm{C}(016)-\mathrm{C}(019)$ & $1.371(3)$ \\
\hline $\mathrm{C}(017)-\mathrm{C}(01 \mathrm{E})$ & $1.388(3)$ \\
\hline $\mathrm{C}(017)-\mathrm{C}(01 \mathrm{P})$ & $1.409(3)$ \\
\hline $\mathrm{C}(018)-\mathrm{C}(01 \mathrm{G})$ & $1.369(4)$ \\
\hline $\mathrm{C}(019)-\mathrm{C}(01 \mathrm{D})$ & $1.406(3)$ \\
\hline $\mathrm{C}(01 \mathrm{~A})-\mathrm{C}(01 \mathrm{~K})$ & $1.362(3)$ \\
\hline $\mathrm{C}(01 \mathrm{~B})-\mathrm{C}(01 \mathrm{~L})$ & 1.391(3) \\
\hline $\mathrm{C}(01 \mathrm{~B})-\mathrm{C}(01 \mathrm{M})$ & $1.398(3)$ \\
\hline $\mathrm{C}(01 \mathrm{C})-\mathrm{C}(01 \mathrm{~J})$ & 1.391(3) \\
\hline C(01E)-H(01E) & 0.9500 \\
\hline$C(01 E)-C(01 N)$ & $1.401(3)$ \\
\hline $\mathrm{C}(01 \mathrm{~F})-\mathrm{C}(01 \mathrm{~N})$ & $1.398(3)$ \\
\hline $\mathrm{C}(01 \mathrm{~F})-\mathrm{C}(01 \mathrm{O})$ & $1.399(3)$ \\
\hline $\mathrm{C}(01 \mathrm{G})-\mathrm{C}(01 \mathrm{~K})$ & $1.398(4)$ \\
\hline $\mathrm{C}(01 \mathrm{H})-\mathrm{C}(01 \mathrm{~S})$ & $1.364(4)$ \\
\hline $\mathrm{C}(01 \mathrm{I})-\mathrm{C}(01 \mathrm{Q})$ & $1.388(3)$ \\
\hline
\end{tabular}




\begin{tabular}{|c|c|}
\hline $\mathrm{C}(01 \mathrm{I})-\mathrm{C}(01 \mathrm{~T})$ & $1.409(3)$ \\
\hline $\mathrm{C}(01 \mathrm{~J})-\mathrm{H}(01 \mathrm{U})$ & 0.9500 \\
\hline $\mathrm{C}(01 \mathrm{~L})-\mathrm{H}(01 \mathrm{~L})$ & 0.9500 \\
\hline $\mathrm{C}(01 \mathrm{~L})-\mathrm{C}(01 \mathrm{Q})$ & $1.406(4)$ \\
\hline $\mathrm{C}(01 \mathrm{M})-\mathrm{H}(01 \mathrm{M})$ & 0.9500 \\
\hline $\mathrm{C}(01 \mathrm{M})-\mathrm{C}(01 \mathrm{~T})$ & $1.383(3)$ \\
\hline $\mathrm{C}(01 \mathrm{~N})-\mathrm{H}(01 \mathrm{~N})$ & 0.9500 \\
\hline $\mathrm{C}(01 \mathrm{O})-\mathrm{H}(01 \mathrm{O})$ & 0.9500 \\
\hline $\mathrm{C}(01 \mathrm{O})-\mathrm{C}(01 \mathrm{P})$ & $1.384(3)$ \\
\hline $\mathrm{C}(01 \mathrm{P})-\mathrm{H}(01 \mathrm{P})$ & 0.9500 \\
\hline C(01Q)-H(01Q) & 0.9500 \\
\hline $\mathrm{C}(01 \mathrm{R})-\mathrm{C}(01 \mathrm{~S})$ & $1.398(4)$ \\
\hline $\mathrm{C}(01 \mathrm{~T})-\mathrm{H}(01 \mathrm{~T})$ & 0.9500 \\
\hline $\mathrm{C}(01 \mathrm{U})-\mathrm{H}(01 \mathrm{~A})$ & 0.9800 \\
\hline $\mathrm{C}(01 \mathrm{U})-\mathrm{H}(01 \mathrm{~B})$ & 0.9800 \\
\hline $\mathrm{C}(01 \mathrm{U})-\mathrm{H}(01 \mathrm{C})$ & 0.9800 \\
\hline $\mathrm{C}(01 \mathrm{~V})-\mathrm{H}(01 \mathrm{D})$ & 0.9800 \\
\hline $\mathrm{C}(01 \mathrm{~V})-\mathrm{H}(01 \mathrm{~F})$ & 0.9800 \\
\hline $\mathrm{C}(01 \mathrm{~V})-\mathrm{H}(01 \mathrm{G})$ & 0.9800 \\
\hline $\mathrm{C}(01 \mathrm{~W})-\mathrm{H}(01 \mathrm{~V})$ & 0.9800 \\
\hline $\mathrm{C}(01 \mathrm{~W})-\mathrm{H}(01 \mathrm{~W})$ & 0.9800 \\
\hline $\mathrm{C}(01 \mathrm{~W})-\mathrm{H}(01 \mathrm{X})$ & 0.9800 \\
\hline $\mathrm{C}(01 \mathrm{X})-\mathrm{H}(01 \mathrm{H})$ & 0.9800 \\
\hline $\mathrm{C}(01 \mathrm{X})-\mathrm{H}(01 \mathrm{I})$ & 0.9800 \\
\hline $\mathrm{C}(01 \mathrm{X})-\mathrm{H}(01 \mathrm{~J})$ & 0.9800 \\
\hline $\mathrm{C}(01 \mathrm{Y})-\mathrm{H}(01 \mathrm{~K})$ & 0.9800 \\
\hline $\mathrm{C}(01 \mathrm{Y})-\mathrm{H}(01 \mathrm{R})$ & 0.9800 \\
\hline $\mathrm{C}(01 \mathrm{Y})-\mathrm{H}(01 \mathrm{~S})$ & 0.9800 \\
\hline $\mathrm{C}(01 \mathrm{~F})-\mathrm{O}(00 \mathrm{~L})-\mathrm{C}(01 \mathrm{U})$ & $117.23(18)$ \\
\hline $\mathrm{C}(01 \mathrm{C})-\mathrm{O}(00 \mathrm{M})-\mathrm{C}(01 \mathrm{~W})$ & $117.46(18)$ \\
\hline $\mathrm{C}(017)-\mathrm{O}(00 \mathrm{~N})-\mathrm{C}(01 \mathrm{~V})$ & $116.49(19)$ \\
\hline $\mathrm{C}(01 \mathrm{~B})-\mathrm{O}(00 \mathrm{O})-\mathrm{C}(01 \mathrm{Y})$ & $117.0(2)$ \\
\hline $\mathrm{C}(01 \mathrm{I})-\mathrm{O}(00 \mathrm{P})-\mathrm{C}(01 \mathrm{X})$ & $117.1(2)$ \\
\hline $\mathrm{C}(00 \mathrm{R}) \# 1-\mathrm{C}(00 \mathrm{Q})-\mathrm{C}(00 \mathrm{Q}) \# 1$ & $118.7(2)$ \\
\hline $\mathrm{C}(00 \mathrm{R}) \# 1-\mathrm{C}(00 \mathrm{Q})-\mathrm{C}(00 \mathrm{~S})$ & $123.18(19)$ \\
\hline
\end{tabular}




\begin{tabular}{|c|c|}
\hline $\mathrm{C}(00 \mathrm{~S})-\mathrm{C}(00 \mathrm{Q})-\mathrm{C}(00 \mathrm{Q}) \# 1$ & $118.1(2)$ \\
\hline $\mathrm{F}(002)-\mathrm{C}(00 \mathrm{R})-\mathrm{C}(00 \mathrm{Q}) \# 1$ & $120.53(18)$ \\
\hline $\mathrm{F}(002)-\mathrm{C}(00 \mathrm{R})-\mathrm{C}(00 \mathrm{U})$ & $118.46(19)$ \\
\hline $\mathrm{C}(00 \mathrm{U})-\mathrm{C}(00 \mathrm{R})-\mathrm{C}(00 \mathrm{Q}) \# 1$ & $121.00(19)$ \\
\hline $\mathrm{F}(001)-\mathrm{C}(00 \mathrm{~S})-\mathrm{C}(00 \mathrm{Q})$ & $120.58(19)$ \\
\hline $\mathrm{F}(001)-\mathrm{C}(00 \mathrm{~S})-\mathrm{C}(00 \mathrm{Z})$ & $117.95(18)$ \\
\hline $\mathrm{C}(00 \mathrm{Z})-\mathrm{C}(00 \mathrm{~S})-\mathrm{C}(00 \mathrm{Q})$ & $121.46(19)$ \\
\hline $\mathrm{C}(010)-\mathrm{C}(00 \mathrm{~T})-\mathrm{C}(012)$ & $118.48(19)$ \\
\hline $\mathrm{C}(016)-\mathrm{C}(00 \mathrm{~T})-\mathrm{C}(010)$ & $123.3(2)$ \\
\hline $\mathrm{C}(016)-\mathrm{C}(00 \mathrm{~T})-\mathrm{C}(012)$ & $118.3(2)$ \\
\hline $\mathrm{F}(008)-\mathrm{C}(00 \mathrm{U})-\mathrm{C}(00 \mathrm{R})$ & $120.65(19)$ \\
\hline $\mathrm{F}(008)-\mathrm{C}(00 \mathrm{U})-\mathrm{C}(00 \mathrm{Z})$ & 118.97(18) \\
\hline $\mathrm{C}(00 \mathrm{R})-\mathrm{C}(00 \mathrm{U})-\mathrm{C}(00 \mathrm{Z})$ & $120.4(2)$ \\
\hline $\mathrm{F}(007)-\mathrm{C}(00 \mathrm{~V})-\mathrm{C}(012)$ & $120.51(19)$ \\
\hline $\mathrm{F}(007)-\mathrm{C}(00 \mathrm{~V})-\mathrm{C}(01 \mathrm{D})$ & $118.4(2)$ \\
\hline $\mathrm{C}(01 \mathrm{D})-\mathrm{C}(00 \mathrm{~V})-\mathrm{C}(012)$ & $121.1(2)$ \\
\hline $\mathrm{C}(00 \mathrm{X})-\mathrm{C}(00 \mathrm{~W})-\mathrm{C}(015)$ & $118.3(2)$ \\
\hline $\mathrm{C}(00 \mathrm{X})-\mathrm{C}(00 \mathrm{~W})-\mathrm{C}(01 \mathrm{~A})$ & $123.7(2)$ \\
\hline $\mathrm{C}(01 \mathrm{~A})-\mathrm{C}(00 \mathrm{~W})-\mathrm{C}(015)$ & $118.1(2)$ \\
\hline $\mathrm{F}(009)-\mathrm{C}(00 \mathrm{X})-\mathrm{C}(00 \mathrm{~W})$ & $120.0(2)$ \\
\hline $\mathrm{F}(009)-\mathrm{C}(00 \mathrm{X})-\mathrm{C}(01 \mathrm{R})$ & $118.2(2)$ \\
\hline $\mathrm{C}(01 \mathrm{R})-\mathrm{C}(00 \mathrm{X})-\mathrm{C}(00 \mathrm{~W})$ & $121.8(2)$ \\
\hline $\mathrm{F}(00 \mathrm{C})-\mathrm{C}(00 \mathrm{Y})-\mathrm{C}(011)$ & $118.9(2)$ \\
\hline $\mathrm{F}(00 \mathrm{C})-\mathrm{C}(00 \mathrm{Y})-\mathrm{C}(014)$ & $120.9(2)$ \\
\hline $\mathrm{C}(014)-\mathrm{C}(00 \mathrm{Y})-\mathrm{C}(011)$ & $120.2(2)$ \\
\hline $\mathrm{F}(004)-\mathrm{C}(00 \mathrm{Z})-\mathrm{C}(00 \mathrm{~S})$ & $120.58(19)$ \\
\hline $\mathrm{F}(004)-\mathrm{C}(00 \mathrm{Z})-\mathrm{C}(00 \mathrm{U})$ & $119.07(19)$ \\
\hline $\mathrm{C}(00 \mathrm{~S})-\mathrm{C}(00 \mathrm{Z})-\mathrm{C}(00 \mathrm{U})$ & $120.35(19)$ \\
\hline $\mathrm{F}(006)-\mathrm{C}(010)-\mathrm{C}(00 \mathrm{~T})$ & $120.12(19)$ \\
\hline $\mathrm{F}(006)-\mathrm{C}(010)-\mathrm{C}(011)$ & $118.6(2)$ \\
\hline $\mathrm{C}(011)-\mathrm{C}(010)-\mathrm{C}(00 \mathrm{~T})$ & $121.3(2)$ \\
\hline $\mathrm{F}(00 \mathrm{E})-\mathrm{C}(011)-\mathrm{C}(00 \mathrm{Y})$ & $118.6(2)$ \\
\hline $\mathrm{F}(00 \mathrm{E})-\mathrm{C}(011)-\mathrm{C}(010)$ & $121.0(2)$ \\
\hline $\mathrm{C}(010)-\mathrm{C}(011)-\mathrm{C}(00 \mathrm{Y})$ & $120.4(2)$ \\
\hline $\mathrm{C}(00 \mathrm{~V})-\mathrm{C}(012)-\mathrm{C}(00 \mathrm{~T})$ & $118.6(2)$ \\
\hline $\mathrm{C}(00 \mathrm{~V})-\mathrm{C}(012)-\mathrm{C}(014)$ & $123.3(2)$ \\
\hline
\end{tabular}




\begin{tabular}{|c|c|}
\hline $\mathrm{C}(014)-\mathrm{C}(012)-\mathrm{C}(00 \mathrm{~T})$ & $118.0(2)$ \\
\hline $\mathrm{C}(01 \mathrm{C})-\mathrm{C}(013)-\mathrm{H}(013)$ & 120.3 \\
\hline $\mathrm{C}(01 \mathrm{~J}) \# 2-\mathrm{C}(013)-\mathrm{H}(013)$ & 120.3 \\
\hline $\mathrm{C}(01 \mathrm{~J}) \# 2-\mathrm{C}(013)-\mathrm{C}(01 \mathrm{C})$ & $119.5(2)$ \\
\hline $\mathrm{F}(005)-\mathrm{C}(014)-\mathrm{C}(00 \mathrm{Y})$ & $118.01(19)$ \\
\hline $\mathrm{F}(005)-\mathrm{C}(014)-\mathrm{C}(012)$ & $120.4(2)$ \\
\hline $\mathrm{C}(00 \mathrm{Y})-\mathrm{C}(014)-\mathrm{C}(012)$ & $121.6(2)$ \\
\hline $\mathrm{C}(018)-\mathrm{C}(015)-\mathrm{C}(00 \mathrm{~W})$ & $118.4(2)$ \\
\hline $\mathrm{C}(018)-\mathrm{C}(015)-\mathrm{C}(01 \mathrm{H})$ & $123.8(2)$ \\
\hline $\mathrm{C}(01 \mathrm{H})-\mathrm{C}(015)-\mathrm{C}(00 \mathrm{~W})$ & $117.9(2)$ \\
\hline $\mathrm{F}(003)-\mathrm{C}(016)-\mathrm{C}(00 \mathrm{~T})$ & $120.5(2)$ \\
\hline $\mathrm{F}(003)-\mathrm{C}(016)-\mathrm{C}(019)$ & $117.88(19)$ \\
\hline $\mathrm{C}(019)-\mathrm{C}(016)-\mathrm{C}(00 \mathrm{~T})$ & $121.6(2)$ \\
\hline $\mathrm{O}(00 \mathrm{~N})-\mathrm{C}(017)-\mathrm{C}(01 \mathrm{E})$ & $124.7(2)$ \\
\hline $\mathrm{O}(00 \mathrm{~N})-\mathrm{C}(017)-\mathrm{C}(01 \mathrm{P})$ & $115.9(2)$ \\
\hline $\mathrm{C}(01 \mathrm{E})-\mathrm{C}(017)-\mathrm{C}(01 \mathrm{P})$ & $119.4(2)$ \\
\hline $\mathrm{F}(00 \mathrm{G})-\mathrm{C}(018)-\mathrm{C}(015)$ & $120.1(2)$ \\
\hline $\mathrm{F}(00 \mathrm{G})-\mathrm{C}(018)-\mathrm{C}(01 \mathrm{G})$ & $118.6(2)$ \\
\hline $\mathrm{C}(01 \mathrm{G})-\mathrm{C}(018)-\mathrm{C}(015)$ & $121.2(2)$ \\
\hline $\mathrm{F}(00 \mathrm{~B})-\mathrm{C}(019)-\mathrm{C}(016)$ & $121.0(2)$ \\
\hline $\mathrm{F}(00 \mathrm{~B})-\mathrm{C}(019)-\mathrm{C}(01 \mathrm{D})$ & 119.1(2) \\
\hline $\mathrm{C}(016)-\mathrm{C}(019)-\mathrm{C}(01 \mathrm{D})$ & $119.8(2)$ \\
\hline $\mathrm{F}(00 \mathrm{~A})-\mathrm{C}(01 \mathrm{~A})-\mathrm{C}(00 \mathrm{~W})$ & $120.2(2)$ \\
\hline $\mathrm{F}(00 \mathrm{~A})-\mathrm{C}(01 \mathrm{~A})-\mathrm{C}(01 \mathrm{~K})$ & $118.3(2)$ \\
\hline $\mathrm{C}(01 \mathrm{~K})-\mathrm{C}(01 \mathrm{~A})-\mathrm{C}(00 \mathrm{~W})$ & $121.5(2)$ \\
\hline $\mathrm{O}(00 \mathrm{O})-\mathrm{C}(01 \mathrm{~B})-\mathrm{C}(01 \mathrm{~L})$ & $125.4(2)$ \\
\hline $\mathrm{O}(00 \mathrm{O})-\mathrm{C}(01 \mathrm{~B})-\mathrm{C}(01 \mathrm{M})$ & $115.4(2)$ \\
\hline $\mathrm{C}(01 \mathrm{~L})-\mathrm{C}(01 \mathrm{~B})-\mathrm{C}(01 \mathrm{M})$ & 119.1(2) \\
\hline $\mathrm{O}(00 \mathrm{M})-\mathrm{C}(01 \mathrm{C})-\mathrm{C}(013)$ & $125.0(2)$ \\
\hline $\mathrm{O}(00 \mathrm{M})-\mathrm{C}(01 \mathrm{C})-\mathrm{C}(01 \mathrm{~J})$ & $115.54(19)$ \\
\hline $\mathrm{C}(01 \mathrm{~J})-\mathrm{C}(01 \mathrm{C})-\mathrm{C}(013)$ & $119.4(2)$ \\
\hline $\mathrm{F}(00 \mathrm{D})-\mathrm{C}(01 \mathrm{D})-\mathrm{C}(00 \mathrm{~V})$ & $121.0(2)$ \\
\hline $\mathrm{F}(00 \mathrm{D})-\mathrm{C}(01 \mathrm{D})-\mathrm{C}(019)$ & $118.5(2)$ \\
\hline $\mathrm{C}(00 \mathrm{~V})-\mathrm{C}(01 \mathrm{D})-\mathrm{C}(019)$ & $120.5(2)$ \\
\hline $\mathrm{C}(017)-\mathrm{C}(01 \mathrm{E})-\mathrm{H}(01 \mathrm{E})$ & 119.8 \\
\hline $\mathrm{C}(017)-\mathrm{C}(01 \mathrm{E})-\mathrm{C}(01 \mathrm{~N})$ & $120.4(2)$ \\
\hline
\end{tabular}




\begin{tabular}{|c|c|}
\hline $\mathrm{C}(01 \mathrm{~N})-\mathrm{C}(01 \mathrm{E})-\mathrm{H}(01 \mathrm{E})$ & 119.8 \\
\hline $\mathrm{O}(00 \mathrm{~L})-\mathrm{C}(01 \mathrm{~F})-\mathrm{C}(01 \mathrm{~N})$ & $124.9(2)$ \\
\hline $\mathrm{O}(00 \mathrm{~L})-\mathrm{C}(01 \mathrm{~F})-\mathrm{C}(01 \mathrm{O})$ & 115.77(19) \\
\hline $\mathrm{C}(01 \mathrm{~N})-\mathrm{C}(01 \mathrm{~F})-\mathrm{C}(01 \mathrm{O})$ & $119.4(2)$ \\
\hline $\mathrm{F}(00 \mathrm{I})-\mathrm{C}(01 \mathrm{G})-\mathrm{C}(018)$ & $121.0(2)$ \\
\hline $\mathrm{F}(00 \mathrm{I})-\mathrm{C}(01 \mathrm{G})-\mathrm{C}(01 \mathrm{~K})$ & $118.8(2)$ \\
\hline $\mathrm{C}(018)-\mathrm{C}(01 \mathrm{G})-\mathrm{C}(01 \mathrm{~K})$ & $120.2(2)$ \\
\hline $\mathrm{F}(00 \mathrm{~F})-\mathrm{C}(01 \mathrm{H})-\mathrm{C}(015)$ & $119.8(2)$ \\
\hline $\mathrm{F}(00 \mathrm{~F})-\mathrm{C}(01 \mathrm{H})-\mathrm{C}(01 \mathrm{~S})$ & $118.5(2)$ \\
\hline $\mathrm{C}(01 \mathrm{~S})-\mathrm{C}(01 \mathrm{H})-\mathrm{C}(015)$ & $121.7(2)$ \\
\hline $\mathrm{O}(00 \mathrm{P})-\mathrm{C}(01 \mathrm{I})-\mathrm{C}(01 \mathrm{Q})$ & $124.9(2)$ \\
\hline $\mathrm{O}(00 \mathrm{P})-\mathrm{C}(01 \mathrm{I})-\mathrm{C}(01 \mathrm{~T})$ & $115.7(2)$ \\
\hline $\mathrm{C}(01 \mathrm{Q})-\mathrm{C}(01 \mathrm{I})-\mathrm{C}(01 \mathrm{~T})$ & $119.4(2)$ \\
\hline $\mathrm{C}(013) \# 2-\mathrm{C}(01 \mathrm{~J})-\mathrm{H}(01 \mathrm{U})$ & 119.5 \\
\hline $\mathrm{C}(01 \mathrm{C})-\mathrm{C}(01 \mathrm{~J})-\mathrm{C}(013) \# 2$ & $121.1(2)$ \\
\hline $\mathrm{C}(01 \mathrm{C})-\mathrm{C}(01 \mathrm{~J})-\mathrm{H}(01 \mathrm{U})$ & 119.5 \\
\hline $\mathrm{F}(00 \mathrm{H})-\mathrm{C}(01 \mathrm{~K})-\mathrm{C}(01 \mathrm{~A})$ & $120.3(2)$ \\
\hline $\mathrm{F}(00 \mathrm{H})-\mathrm{C}(01 \mathrm{~K})-\mathrm{C}(01 \mathrm{G})$ & $119.1(2)$ \\
\hline $\mathrm{C}(01 \mathrm{~A})-\mathrm{C}(01 \mathrm{~K})-\mathrm{C}(01 \mathrm{G})$ & $120.6(2)$ \\
\hline $\mathrm{C}(01 \mathrm{~B})-\mathrm{C}(01 \mathrm{~L})-\mathrm{H}(01 \mathrm{~L})$ & 119.8 \\
\hline $\mathrm{C}(01 \mathrm{~B})-\mathrm{C}(01 \mathrm{~L})-\mathrm{C}(01 \mathrm{Q})$ & $120.4(2)$ \\
\hline $\mathrm{C}(01 \mathrm{Q})-\mathrm{C}(01 \mathrm{~L})-\mathrm{H}(01 \mathrm{~L})$ & 119.8 \\
\hline $\mathrm{C}(01 \mathrm{~B})-\mathrm{C}(01 \mathrm{M})-\mathrm{H}(01 \mathrm{M})$ & 119.5 \\
\hline $\mathrm{C}(01 \mathrm{~T})-\mathrm{C}(01 \mathrm{M})-\mathrm{C}(01 \mathrm{~B})$ & $120.9(2)$ \\
\hline $\mathrm{C}(01 \mathrm{~T})-\mathrm{C}(01 \mathrm{M})-\mathrm{H}(01 \mathrm{M})$ & 119.5 \\
\hline $\mathrm{C}(01 \mathrm{E})-\mathrm{C}(01 \mathrm{~N})-\mathrm{H}(01 \mathrm{~N})$ & 120.0 \\
\hline $\mathrm{C}(01 \mathrm{~F})-\mathrm{C}(01 \mathrm{~N})-\mathrm{C}(01 \mathrm{E})$ & $120.0(2)$ \\
\hline $\mathrm{C}(01 \mathrm{~F})-\mathrm{C}(01 \mathrm{~N})-\mathrm{H}(01 \mathrm{~N})$ & 120.0 \\
\hline $\mathrm{C}(01 \mathrm{~F})-\mathrm{C}(01 \mathrm{O})-\mathrm{H}(01 \mathrm{O})$ & 119.7 \\
\hline $\mathrm{C}(01 \mathrm{P})-\mathrm{C}(01 \mathrm{O})-\mathrm{C}(01 \mathrm{~F})$ & $120.6(2)$ \\
\hline $\mathrm{C}(01 \mathrm{P})-\mathrm{C}(01 \mathrm{O})-\mathrm{H}(01 \mathrm{O})$ & 119.7 \\
\hline $\mathrm{C}(017)-\mathrm{C}(01 \mathrm{P})-\mathrm{H}(01 \mathrm{P})$ & 119.9 \\
\hline $\mathrm{C}(01 \mathrm{O})-\mathrm{C}(01 \mathrm{P})-\mathrm{C}(017)$ & $120.1(2)$ \\
\hline $\mathrm{C}(01 \mathrm{O})-\mathrm{C}(01 \mathrm{P})-\mathrm{H}(01 \mathrm{P})$ & 119.9 \\
\hline $\mathrm{C}(01 \mathrm{I})-\mathrm{C}(01 \mathrm{Q})-\mathrm{C}(01 \mathrm{~L})$ & $120.1(2)$ \\
\hline C(01I)-C(01Q)-H(01Q) & 119.9 \\
\hline
\end{tabular}




\begin{tabular}{|c|c|}
\hline $\mathrm{C}(01 \mathrm{~L})-\mathrm{C}(01 \mathrm{Q})-\mathrm{H}(01 \mathrm{Q})$ & 119.9 \\
\hline $\mathrm{F}(00 \mathrm{~J})-\mathrm{C}(01 \mathrm{R})-\mathrm{C}(00 \mathrm{X})$ & $120.8(2)$ \\
\hline $\mathrm{F}(00 \mathrm{~J})-\mathrm{C}(01 \mathrm{R})-\mathrm{C}(01 \mathrm{~S})$ & $119.4(2)$ \\
\hline $\mathrm{C}(00 \mathrm{X})-\mathrm{C}(01 \mathrm{R})-\mathrm{C}(01 \mathrm{~S})$ & $119.8(2)$ \\
\hline $\mathrm{F}(00 \mathrm{~K})-\mathrm{C}(01 \mathrm{~S})-\mathrm{C}(01 \mathrm{H})$ & $120.1(2)$ \\
\hline $\mathrm{F}(00 \mathrm{~K})-\mathrm{C}(01 \mathrm{~S})-\mathrm{C}(01 \mathrm{R})$ & $119.2(2)$ \\
\hline $\mathrm{C}(01 \mathrm{H})-\mathrm{C}(01 \mathrm{~S})-\mathrm{C}(01 \mathrm{R})$ & $120.6(2)$ \\
\hline $\mathrm{C}(01 \mathrm{I})-\mathrm{C}(01 \mathrm{~T})-\mathrm{H}(01 \mathrm{~T})$ & 120.0 \\
\hline $\mathrm{C}(01 \mathrm{M})-\mathrm{C}(01 \mathrm{~T})-\mathrm{C}(01 \mathrm{I})$ & $120.0(2)$ \\
\hline $\mathrm{C}(01 \mathrm{M})-\mathrm{C}(01 \mathrm{~T})-\mathrm{H}(01 \mathrm{~T})$ & 120.0 \\
\hline $\mathrm{O}(00 \mathrm{~L})-\mathrm{C}(01 \mathrm{U})-\mathrm{H}(01 \mathrm{~A})$ & 109.5 \\
\hline $\mathrm{O}(00 \mathrm{~L})-\mathrm{C}(01 \mathrm{U})-\mathrm{H}(01 \mathrm{~B})$ & 109.5 \\
\hline $\mathrm{O}(00 \mathrm{~L})-\mathrm{C}(01 \mathrm{U})-\mathrm{H}(01 \mathrm{C})$ & 109.5 \\
\hline $\mathrm{H}(01 \mathrm{~A})-\mathrm{C}(01 \mathrm{U})-\mathrm{H}(01 \mathrm{~B})$ & 109.5 \\
\hline $\mathrm{H}(01 \mathrm{~A})-\mathrm{C}(01 \mathrm{U})-\mathrm{H}(01 \mathrm{C})$ & 109.5 \\
\hline $\mathrm{H}(01 \mathrm{~B})-\mathrm{C}(01 \mathrm{U})-\mathrm{H}(01 \mathrm{C})$ & 109.5 \\
\hline $\mathrm{O}(00 \mathrm{~N})-\mathrm{C}(01 \mathrm{~V})-\mathrm{H}(01 \mathrm{D})$ & 109.5 \\
\hline $\mathrm{O}(00 \mathrm{~N})-\mathrm{C}(01 \mathrm{~V})-\mathrm{H}(01 \mathrm{~F})$ & 109.5 \\
\hline $\mathrm{O}(00 \mathrm{~N})-\mathrm{C}(01 \mathrm{~V})-\mathrm{H}(01 \mathrm{G})$ & 109.5 \\
\hline $\mathrm{H}(01 \mathrm{D})-\mathrm{C}(01 \mathrm{~V})-\mathrm{H}(01 \mathrm{~F})$ & 109.5 \\
\hline $\mathrm{H}(01 \mathrm{D})-\mathrm{C}(01 \mathrm{~V})-\mathrm{H}(01 \mathrm{G})$ & 109.5 \\
\hline $\mathrm{H}(01 \mathrm{~F})-\mathrm{C}(01 \mathrm{~V})-\mathrm{H}(01 \mathrm{G})$ & 109.5 \\
\hline $\mathrm{O}(00 \mathrm{M})-\mathrm{C}(01 \mathrm{~W})-\mathrm{H}(01 \mathrm{~V})$ & 109.5 \\
\hline $\mathrm{O}(00 \mathrm{M})-\mathrm{C}(01 \mathrm{~W})-\mathrm{H}(01 \mathrm{~W})$ & 109.5 \\
\hline $\mathrm{O}(00 \mathrm{M})-\mathrm{C}(01 \mathrm{~W})-\mathrm{H}(01 \mathrm{X})$ & 109.5 \\
\hline $\mathrm{H}(01 \mathrm{~V})-\mathrm{C}(01 \mathrm{~W})-\mathrm{H}(01 \mathrm{~W})$ & 109.5 \\
\hline $\mathrm{H}(01 \mathrm{~V})-\mathrm{C}(01 \mathrm{~W})-\mathrm{H}(01 \mathrm{X})$ & 109.5 \\
\hline $\mathrm{H}(01 \mathrm{~W})-\mathrm{C}(01 \mathrm{~W})-\mathrm{H}(01 \mathrm{X})$ & 109.5 \\
\hline $\mathrm{O}(00 \mathrm{P})-\mathrm{C}(01 \mathrm{X})-\mathrm{H}(01 \mathrm{H})$ & 109.5 \\
\hline $\mathrm{O}(00 \mathrm{P})-\mathrm{C}(01 \mathrm{X})-\mathrm{H}(01 \mathrm{I})$ & 109.5 \\
\hline $\mathrm{O}(00 \mathrm{P})-\mathrm{C}(01 \mathrm{X})-\mathrm{H}(01 \mathrm{~J})$ & 109.5 \\
\hline $\mathrm{H}(01 \mathrm{H})-\mathrm{C}(01 \mathrm{X})-\mathrm{H}(01 \mathrm{I})$ & 109.5 \\
\hline $\mathrm{H}(01 \mathrm{H})-\mathrm{C}(01 \mathrm{X})-\mathrm{H}(01 \mathrm{~J})$ & 109.5 \\
\hline $\mathrm{H}(01 \mathrm{I})-\mathrm{C}(01 \mathrm{X})-\mathrm{H}(01 \mathrm{~J})$ & 109.5 \\
\hline $\mathrm{O}(00 \mathrm{O})-\mathrm{C}(01 \mathrm{Y})-\mathrm{H}(01 \mathrm{~K})$ & 109.5 \\
\hline $\mathrm{O}(00 \mathrm{O})-\mathrm{C}(01 \mathrm{Y})-\mathrm{H}(01 \mathrm{R})$ & 109.5 \\
\hline
\end{tabular}




$\begin{array}{ll}\text { O(00O)-C(01Y)-H(01S) } & 109.5 \\ \text { H(01K)-C(01Y)-H(01R) } & 109.5 \\ \text { H(01K)-C(01Y)-H(01S) } & 109.5 \\ \text { H(01R)-C(01Y)-H(01S) } & 109.5\end{array}$

Symmetry transformations used to generate equivalent atoms:

$\# 1-x+2,-y+1,-z+1 \quad \# 2-x+2,-y,-z+1$ 
Table S15. Anisotropic displacement parameters $\left(\AA^{2} \times 10^{3}\right)$ for complex $\mathbf{1 b} \cdot 5 \mathbf{a}$. The anisotropic displacement factor exponent takes the form: $-2 \pi^{2}\left[h^{2} a^{* 2} U^{11}+\ldots+2 h k a^{*} b^{*} U^{12}\right]$

\begin{tabular}{|c|c|c|c|c|c|c|}
\hline & $\mathrm{U}^{11}$ & $\mathrm{U}^{22}$ & $\mathrm{U}^{33}$ & $U^{23}$ & $\mathrm{U}^{13}$ & $\mathrm{U}^{12}$ \\
\hline $\mathrm{F}(001)$ & $11(1)$ & $17(1)$ & $23(1)$ & $0(1)$ & $-1(1)$ & $-3(1)$ \\
\hline $\mathrm{F}(002)$ & $14(1)$ & $22(1)$ & $18(1)$ & $1(1)$ & $-5(1)$ & $-1(1)$ \\
\hline $\mathrm{F}(003)$ & $11(1)$ & $18(1)$ & $33(1)$ & $-2(1)$ & $2(1)$ & $-2(1)$ \\
\hline $\mathrm{F}(004)$ & $18(1)$ & $19(1)$ & $23(1)$ & $5(1)$ & $7(1)$ & $-3(1)$ \\
\hline $\mathrm{F}(005)$ & $13(1)$ & $20(1)$ & $32(1)$ & $-4(1)$ & $0(1)$ & $-4(1)$ \\
\hline $\mathrm{F}(006)$ & $15(1)$ & $27(1)$ & $24(1)$ & $-3(1)$ & $-7(1)$ & $0(1)$ \\
\hline $\mathrm{F}(007)$ & $17(1)$ & $29(1)$ & $24(1)$ & $-4(1)$ & $-7(1)$ & $2(1)$ \\
\hline $\mathrm{F}(008)$ & $23(1)$ & $24(1)$ & $13(1)$ & $5(1)$ & $0(1)$ & $2(1)$ \\
\hline $\mathrm{F}(009)$ & $22(1)$ & $34(1)$ & $27(1)$ & $-7(1)$ & $-8(1)$ & $4(1)$ \\
\hline $\mathrm{F}(00 \mathrm{~A})$ & $14(1)$ & $21(1)$ & $40(1)$ & $-5(1)$ & $1(1)$ & $-5(1)$ \\
\hline $\mathrm{F}(00 \mathrm{~B})$ & $28(1)$ & 19(1) & $32(1)$ & $5(1)$ & $13(1)$ & $-1(1)$ \\
\hline $\mathrm{F}(00 \mathrm{C})$ & $24(1)$ & $20(1)$ & $31(1)$ & $3(1)$ & $11(1)$ & $-2(1)$ \\
\hline $\mathrm{F}(00 \mathrm{D})$ & $34(1)$ & $29(1)$ & $18(1)$ & $4(1)$ & $2(1)$ & $9(1)$ \\
\hline $\mathrm{F}(00 \mathrm{E})$ & $32(1)$ & $27(1)$ & $19(1)$ & $5(1)$ & $0(1)$ & $4(1)$ \\
\hline $\mathrm{F}(00 \mathrm{~F})$ & $16(1)$ & $23(1)$ & $62(1)$ & $-10(1)$ & $5(1)$ & $-6(1)$ \\
\hline $\mathrm{F}(00 \mathrm{G})$ & $22(1)$ & $39(1)$ & $40(1)$ & $-13(1)$ & $-14(1)$ & $4(1)$ \\
\hline $\mathrm{F}(00 \mathrm{H})$ & $46(1)$ & $22(1)$ & $39(1)$ & $9(1)$ & $17(1)$ & $1(1)$ \\
\hline $\mathrm{F}(00 \mathrm{I})$ & $53(1)$ & $40(1)$ & $23(1)$ & $4(1)$ & $-8(1)$ & $16(1)$ \\
\hline $\mathrm{F}(00 \mathrm{~J})$ & $58(1)$ & $39(1)$ & $23(1)$ & $6(1)$ & $8(1)$ & $15(1)$ \\
\hline $\mathrm{F}(00 \mathrm{~K})$ & $48(1)$ & $25(1)$ & $51(1)$ & $3(1)$ & $32(1)$ & $-3(1)$ \\
\hline $\mathrm{O}(00 \mathrm{~L})$ & $20(1)$ & $31(1)$ & $20(1)$ & $4(1)$ & 1(1) & $0(1)$ \\
\hline $\mathrm{O}(00 \mathrm{M})$ & $18(1)$ & $25(1)$ & $25(1)$ & $0(1)$ & $1(1)$ & $-3(1)$ \\
\hline $\mathrm{O}(00 \mathrm{~N})$ & $22(1)$ & $28(1)$ & $22(1)$ & $5(1)$ & 1(1) & $-1(1)$ \\
\hline $\mathrm{O}(00 \mathrm{O})$ & $30(1)$ & $30(1)$ & $23(1)$ & $3(1)$ & $-4(1)$ & $1(1)$ \\
\hline $\mathrm{O}(00 \mathrm{P})$ & $31(1)$ & $28(1)$ & $22(1)$ & $5(1)$ & $1(1)$ & $-6(1)$ \\
\hline $\mathrm{C}(00 \mathrm{Q})$ & $10(1)$ & $9(1)$ & $14(1)$ & $-2(1)$ & $1(1)$ & $0(1)$ \\
\hline $\mathrm{C}(00 \mathrm{R})$ & $11(1)$ & $12(1)$ & $16(1)$ & $-4(1)$ & $-1(1)$ & 2(1) \\
\hline $\mathrm{C}(00 \mathrm{~S})$ & $8(1)$ & $9(1)$ & $18(1)$ & $-3(1)$ & $1(1)$ & $1(1)$ \\
\hline $\mathrm{C}(00 \mathrm{~T})$ & $12(1)$ & $10(1)$ & $20(1)$ & $-3(1)$ & $2(1)$ & $2(1)$ \\
\hline $\mathrm{C}(00 \mathrm{U})$ & $18(1)$ & $14(1)$ & $13(1)$ & $1(1)$ & $2(1)$ & $4(1)$ \\
\hline $\mathrm{C}(00 \mathrm{~V})$ & $13(1)$ & $15(1)$ & $20(1)$ & $-6(1)$ & $-2(1)$ & $3(1)$ \\
\hline
\end{tabular}




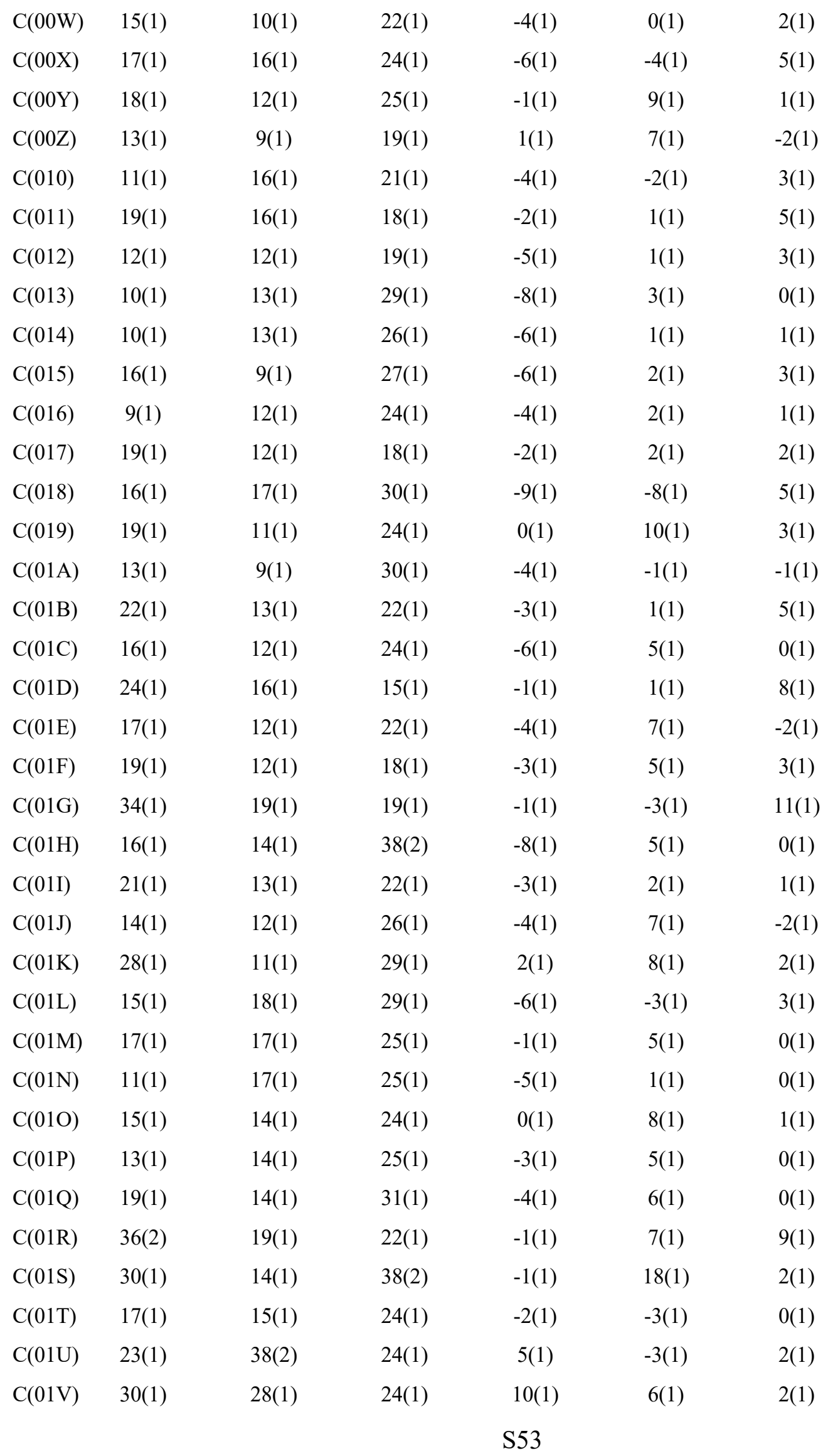




\begin{tabular}{lllllll}
$\mathrm{C}(01 \mathrm{~W})$ & $19(1)$ & $29(1)$ & $26(1)$ & $-3(1)$ & $-1(1)$ & $-2(1)$ \\
$\mathrm{C}(01 \mathrm{X})$ & $39(2)$ & $24(1)$ & $32(1)$ & $4(1)$ & $15(1)$ & $1(1)$ \\
$\mathrm{C}(01 \mathrm{Y})$ & $34(2)$ & $37(2)$ & $26(1)$ & $-2(1)$ & $-9(1)$ & $7(1)$ \\
\hline
\end{tabular}


Table S16. Hydrogen coordinates ( $\left.\times 10^{4}\right)$ and isotropic displacement parameters $\left(\AA^{2} \times 10^{3}\right)$ for complex $\mathbf{1 b} \cdot \mathbf{5 a}$.

\begin{tabular}{|c|c|c|c|c|}
\hline & $\mathrm{x}$ & $\mathrm{y}$ & $\mathrm{z}$ & $\mathrm{U}(\mathrm{eq})$ \\
\hline $\mathrm{H}(013)$ & 13482 & 675 & 5228 & 21 \\
\hline $\mathrm{H}(01 \mathrm{E})$ & 10605 & 9927 & 6762 & 20 \\
\hline $\mathrm{H}(01 \mathrm{U})$ & 7722 & -1678 & 5282 & 21 \\
\hline $\mathrm{H}(01 \mathrm{~L})$ & 8725 & 7765 & 9376 & 26 \\
\hline $\mathrm{H}(01 \mathrm{M})$ & 2604 & 5976 & 9278 & 23 \\
\hline $\mathrm{H}(01 \mathrm{~N})$ & 11612 & 9028 & 7291 & 21 \\
\hline $\mathrm{H}(01 \mathrm{O})$ & 5663 & 6983 & 7340 & 21 \\
\hline $\mathrm{H}(01 \mathrm{P})$ & 4640 & 7927 & 6821 & 21 \\
\hline $\mathrm{H}(01 \mathrm{Q})$ & 8342 & 8713 & 8837 & 25 \\
\hline $\mathrm{H}(01 \mathrm{~T})$ & 2194 & 6937 & 8747 & 23 \\
\hline $\mathrm{H}(01 \mathrm{~A})$ & 12375 & 7199 & 7694 & 43 \\
\hline $\mathrm{H}(01 \mathrm{~B})$ & 11375 & 7119 & 8046 & 43 \\
\hline $\mathrm{H}(01 \mathrm{C})$ & 11441 & 8847 & 7868 & 43 \\
\hline $\mathrm{H}(01 \mathrm{D})$ & 7660 & 10410 & 6013 & 40 \\
\hline $\mathrm{H}(01 \mathrm{~F})$ & 9450 & 9306 & 6192 & 40 \\
\hline $\mathrm{H}(01 \mathrm{G})$ & 9039 & 11166 & 6335 & 40 \\
\hline $\mathrm{H}(01 \mathrm{~V})$ & 14433 & -1179 & 5627 & 38 \\
\hline $\mathrm{H}(01 \mathrm{~W})$ & 13450 & -1374 & 5978 & 38 \\
\hline $\mathrm{H}(01 \mathrm{X})$ & 13358 & 384 & 5810 & 38 \\
\hline $\mathrm{H}(01 \mathrm{H})$ & 6119 & 9472 & 8044 & 46 \\
\hline $\mathrm{H}(01 \mathrm{I})$ & 7746 & 8376 & 8259 & 46 \\
\hline $\mathrm{H}(01 \mathrm{~J})$ & 7123 & 10199 & 8398 & 46 \\
\hline $\mathrm{H}(01 \mathrm{~K})$ & 8887 & 5953 & 9802 & 50 \\
\hline $\mathrm{H}(01 \mathrm{R})$ & 7465 & 6004 & 10116 & 50 \\
\hline $\mathrm{H}(01 \mathrm{~S})$ & 7889 & 7676 & 9931 & 50 \\
\hline
\end{tabular}




\subsection{Crystal data and structure for complex 2b·5a (CCDC number: 2060324)}

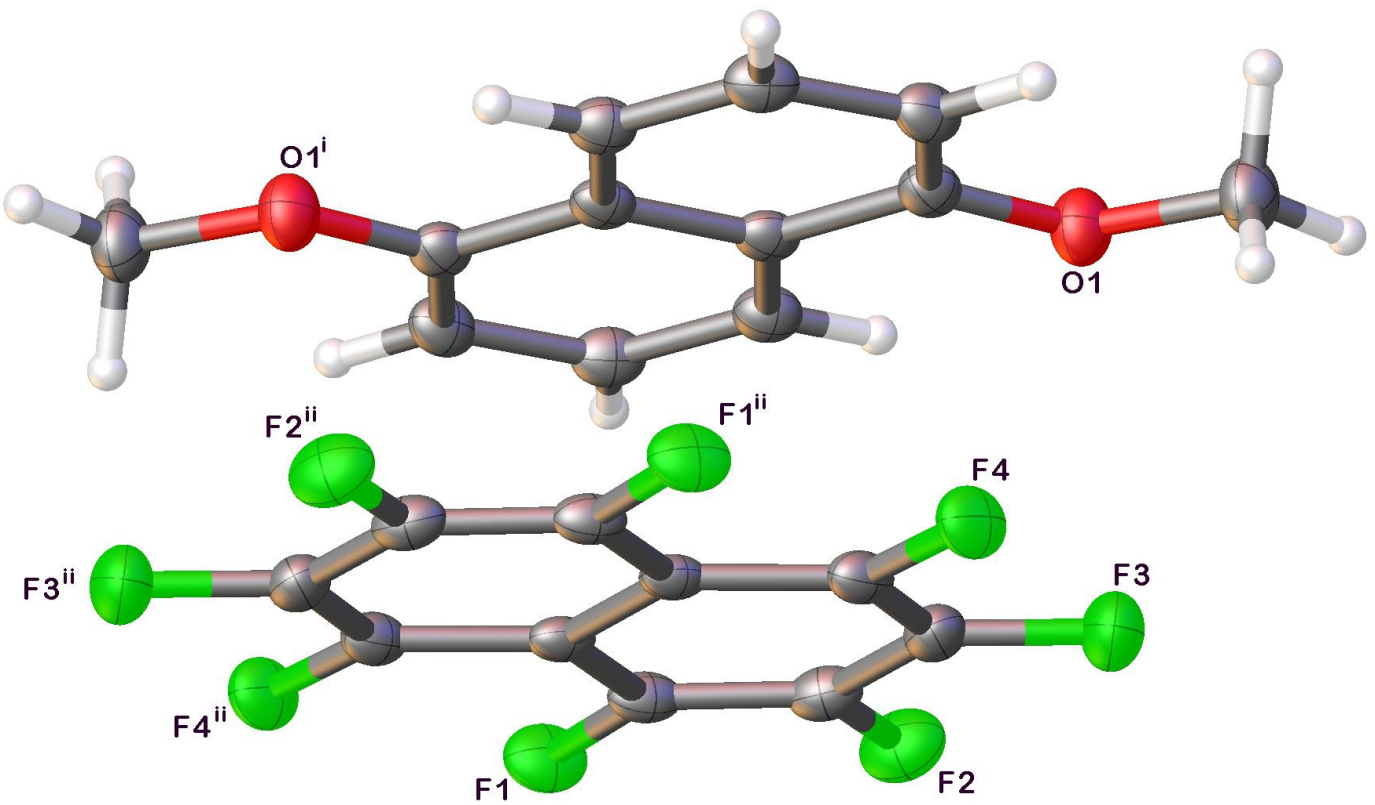

Table S17. Crystal data and structure refinement for complex $\mathbf{2 b} \cdot \mathbf{5 a}$.

Identification code

Empirical formula

Formula weight

Temperature

Wavelength

Crystal system

Space group

Unit cell dimensions

Volume

Z

Density (calculated)

Absorption coefficient

$\mathrm{F}(000)$

Crystal size

Theta range for data collection

Index ranges

Reflections collected

Independent reflections

Completeness to theta $=67.500^{\circ}$

Absorption correction

Max. and min. transmission

Refinement method

Data / restraints / parameters

Goodness-of-fit on $\mathrm{F}^{2}$

Final $\mathrm{R}$ indices [I>2sigma(I)]

$\mathrm{R}$ indices (all data)

Extinction coefficient

Largest diff. peak and hole
GL_03_249 8

$\mathrm{C} 22 \mathrm{H} 12 \mathrm{~F} 8 \mathrm{O} 2$

460.32

$100.0 \mathrm{~K}$

$1.54178 \AA$

Triclinic

P-1

$\mathrm{a}=6.93950(10) \AA$

$\mathrm{b}=8.0953(2) \AA$

$\mathrm{c}=8.3945(2) \AA$

451.591(17) $\AA^{3}$

1

$1.693 \mathrm{Mg} / \mathrm{m}^{3}$

$1.457 \mathrm{~mm}^{-1}$

232

$0.14 \times 0.1 \times 0.03 \mathrm{~mm}^{3}$

5.497 to $71.341^{\circ}$.

$-8<=\mathrm{h}<=8,-9<=\mathrm{k}<=9,-10<=1<=10$

12059

$1695[\mathrm{R}(\mathrm{int})=0.0250]$

$97.8 \%$

None

0.7535 and 0.6498

Full-matrix least-squares on $\mathrm{F}^{2}$

1695 / 0 / 146

1.081

$\mathrm{R} 1=0.0299, \mathrm{wR} 2=0.0814$

$\mathrm{R} 1=0.0317, \mathrm{wR} 2=0.0832$

$\mathrm{n} / \mathrm{a}$

0.258 and -0.165 e. $\AA^{-3}$ $\alpha=97.8300(10)^{\circ}$.

$\beta=104.0430(10)^{\circ}$.

$\gamma=92.7730(10)^{\circ}$. 
Table S18. Atomic coordinates ( x 10 $)$ and equivalent isotropic displacement parameters $\left(\AA^{2} \times 10^{3}\right)$ for complex $\mathbf{2 b} \cdot \mathbf{5 a}$. $\mathrm{U}(\mathrm{eq})$ is defined as one third of the trace of the orthogonalized $\mathrm{U}^{\mathrm{ij}}$ tensor.

\begin{tabular}{lrrrr}
\hline & $\mathrm{x}$ & $\mathrm{y}$ & $\mathrm{z}$ & $\mathrm{U}(\mathrm{eq})$ \\
\hline $\mathrm{F}(1)$ & $10190(1)$ & $8391(1)$ & $8066(1)$ & $31(1)$ \\
$\mathrm{F}(2)$ & $8524(1)$ & $5665(1)$ & $5892(1)$ & $36(1)$ \\
$\mathrm{F}(3)$ & $7728(1)$ & $5700(1)$ & $2599(1)$ & $37(1)$ \\
$\mathrm{F}(4)$ & $8530(1)$ & $8478(1)$ & $1387(1)$ & $32(1)$ \\
$\mathrm{C}(1)$ & $9784(2)$ & $8475(2)$ & $6433(2)$ & $23(1)$ \\
$\mathrm{C}(2)$ & $8959(2)$ & $7079(2)$ & $5350(2)$ & $26(1)$ \\
$\mathrm{C}(3)$ & $8538(2)$ & $7101(2)$ & $3630(2)$ & $26(1)$ \\
$\mathrm{C}(4)$ & $8944(2)$ & $8523(2)$ & $3044(2)$ & $24(1)$ \\
$\mathrm{C}(5)$ & $9782(2)$ & $10012(2)$ & $4123(1)$ & $21(1)$ \\
$\mathrm{O}(1)$ & $3312(1)$ & $6848(1)$ & $2711(1)$ & $29(1)$ \\
$\mathrm{C}(6)$ & $3939(2)$ & $8505(2)$ & $3042(2)$ & $22(1)$ \\
$\mathrm{C}(7)$ & $3913(2)$ & $9529(2)$ & $1854(2)$ & $25(1)$ \\
$\mathrm{C}(8)$ & $4621(2)$ & $11227(2)$ & $2357(2)$ & $25(1)$ \\
$\mathrm{C}(9)$ & $5333(2)$ & $11896(2)$ & $3991(2)$ & $24(1)$ \\
$\mathrm{C}(10)$ & $5350(2)$ & $10855(2)$ & $5232(2)$ & $21(1)$ \\
$\mathrm{C}(11)$ & $2710(2)$ & $6085(2)$ & $1015(2)$ & $32(1)$ \\
& & & & \\
\hline
\end{tabular}


Table S19. Bond lengths $[\AA]$ and angles $\left[{ }^{\circ}\right]$ for complex $\mathbf{2 b} \cdot \mathbf{5 a}$.

\begin{tabular}{|c|c|}
\hline $\mathrm{F}(1)-\mathrm{C}(1)$ & $1.3425(14)$ \\
\hline $\mathrm{F}(2)-\mathrm{C}(2)$ & $1.3355(16)$ \\
\hline $\mathrm{F}(3)-\mathrm{C}(3)$ & $1.3363(15)$ \\
\hline $\mathrm{F}(4)-\mathrm{C}(4)$ & $1.3452(14)$ \\
\hline $\mathrm{C}(1)-\mathrm{C}(2)$ & $1.355(2)$ \\
\hline$C(1)-C(5) \# 1$ & $1.4133(19)$ \\
\hline $\mathrm{C}(2)-\mathrm{C}(3)$ & $1.4049(19)$ \\
\hline$C(3)-C(4)$ & $1.356(2)$ \\
\hline$C(4)-C(5)$ & $1.4112(19)$ \\
\hline$C(5)-C(5) \# 1$ & $1.433(2)$ \\
\hline $\mathrm{O}(1)-\mathrm{C}(6)$ & $1.3599(16)$ \\
\hline $\mathrm{O}(1)-\mathrm{C}(11)$ & $1.4245(16)$ \\
\hline$C(6)-C(7)$ & $1.378(2)$ \\
\hline $\mathrm{C}(6)-\mathrm{C}(10) \# 2$ & $1.4263(18)$ \\
\hline $\mathrm{C}(7)-\mathrm{H}(7)$ & 0.9500 \\
\hline $\mathrm{C}(7)-\mathrm{C}(8)$ & $1.4086(19)$ \\
\hline $\mathrm{C}(8)-\mathrm{H}(8)$ & 0.9500 \\
\hline $\mathrm{C}(8)-\mathrm{C}(9)$ & $1.3644(18)$ \\
\hline $\mathrm{C}(9)-\mathrm{H}(9)$ & 0.9500 \\
\hline $\mathrm{C}(9)-\mathrm{C}(10)$ & $1.4253(19)$ \\
\hline $\mathrm{C}(10)-\mathrm{C}(10) \# 2$ & $1.414(3)$ \\
\hline $\mathrm{C}(11)-\mathrm{H}(11 \mathrm{~A})$ & 0.9800 \\
\hline $\mathrm{C}(11)-\mathrm{H}(11 \mathrm{~B})$ & 0.9800 \\
\hline $\mathrm{C}(11)-\mathrm{H}(11 \mathrm{C})$ & 0.9800 \\
\hline $\mathrm{F}(1)-\mathrm{C}(1)-\mathrm{C}(2)$ & $118.29(12)$ \\
\hline $\mathrm{F}(1)-\mathrm{C}(1)-\mathrm{C}(5) \# 1$ & $120.22(12)$ \\
\hline $\mathrm{C}(2)-\mathrm{C}(1)-\mathrm{C}(5) \# 1$ & $121.49(12)$ \\
\hline $\mathrm{F}(2)-\mathrm{C}(2)-\mathrm{C}(1)$ & $120.89(12)$ \\
\hline $\mathrm{F}(2)-\mathrm{C}(2)-\mathrm{C}(3)$ & $118.82(12)$ \\
\hline $\mathrm{C}(1)-\mathrm{C}(2)-\mathrm{C}(3)$ & $120.28(12)$ \\
\hline $\mathrm{F}(3)-\mathrm{C}(3)-\mathrm{C}(2)$ & $118.64(12)$ \\
\hline $\mathrm{F}(3)-\mathrm{C}(3)-\mathrm{C}(4)$ & $121.23(12)$ \\
\hline $\mathrm{C}(4)-\mathrm{C}(3)-\mathrm{C}(2)$ & $120.13(12)$ \\
\hline
\end{tabular}




\begin{tabular}{|c|c|}
\hline $\mathrm{F}(4)-\mathrm{C}(4)-\mathrm{C}(3)$ & $117.94(12)$ \\
\hline $\mathrm{F}(4)-\mathrm{C}(4)-\mathrm{C}(5)$ & $120.42(12)$ \\
\hline$C(3)-C(4)-C(5)$ & $121.64(12)$ \\
\hline $\mathrm{C}(1) \# 1-\mathrm{C}(5)-\mathrm{C}(5) \# 1$ & $118.25(15)$ \\
\hline $\mathrm{C}(4)-\mathrm{C}(5)-\mathrm{C}(1) \# 1$ & $123.55(12)$ \\
\hline$C(4)-C(5)-C(5) \# 1$ & $118.20(15)$ \\
\hline $\mathrm{C}(6)-\mathrm{O}(1)-\mathrm{C}(11)$ & $117.82(11)$ \\
\hline $\mathrm{O}(1)-\mathrm{C}(6)-\mathrm{C}(7)$ & $124.73(11)$ \\
\hline $\mathrm{O}(1)-\mathrm{C}(6)-\mathrm{C}(10) \# 2$ & $114.32(11)$ \\
\hline $\mathrm{C}(7)-\mathrm{C}(6)-\mathrm{C}(10) \# 2$ & $120.94(12)$ \\
\hline $\mathrm{C}(6)-\mathrm{C}(7)-\mathrm{H}(7)$ & 120.3 \\
\hline $\mathrm{C}(6)-\mathrm{C}(7)-\mathrm{C}(8)$ & $119.30(12)$ \\
\hline $\mathrm{C}(8)-\mathrm{C}(7)-\mathrm{H}(7)$ & 120.3 \\
\hline $\mathrm{C}(7)-\mathrm{C}(8)-\mathrm{H}(8)$ & 119.0 \\
\hline $\mathrm{C}(9)-\mathrm{C}(8)-\mathrm{C}(7)$ & $122.01(12)$ \\
\hline $\mathrm{C}(9)-\mathrm{C}(8)-\mathrm{H}(8)$ & 119.0 \\
\hline $\mathrm{C}(8)-\mathrm{C}(9)-\mathrm{H}(9)$ & 120.4 \\
\hline C(8)-C(9)-C(10) & $119.24(13)$ \\
\hline $\mathrm{C}(10)-\mathrm{C}(9)-\mathrm{H}(9)$ & 120.4 \\
\hline $\mathrm{C}(9)-\mathrm{C}(10)-\mathrm{C}(6) \# 2$ & $121.51(12)$ \\
\hline $\mathrm{C}(10) \# 2-\mathrm{C}(10)-\mathrm{C}(6) \# 2$ & $118.38(15)$ \\
\hline C(10)\#2-C(10)-C(9) & $120.11(14)$ \\
\hline $\mathrm{O}(1)-\mathrm{C}(11)-\mathrm{H}(11 \mathrm{~A})$ & 109.5 \\
\hline $\mathrm{O}(1)-\mathrm{C}(11)-\mathrm{H}(11 \mathrm{~B})$ & 109.5 \\
\hline $\mathrm{O}(1)-\mathrm{C}(11)-\mathrm{H}(11 \mathrm{C})$ & 109.5 \\
\hline $\mathrm{H}(11 \mathrm{~A})-\mathrm{C}(11)-\mathrm{H}(11 \mathrm{~B})$ & 109.5 \\
\hline $\mathrm{H}(11 \mathrm{~A})-\mathrm{C}(11)-\mathrm{H}(11 \mathrm{C})$ & 109.5 \\
\hline $\mathrm{H}(11 \mathrm{~B})-\mathrm{C}(11)-\mathrm{H}(11 \mathrm{C})$ & 109.5 \\
\hline
\end{tabular}

Symmetry transformations used to generate equivalent atoms:

$\# 1-x+2,-y+2,-z+1 \quad \# 2-x+1,-y+2,-z+1$ 
Table S20. Anisotropic displacement parameters $\left(\AA^{2} \times 10^{3}\right)$ for complex 2b·5a. The anisotropic displacement factor exponent takes the form: $-2 \pi^{2}\left[h^{2} a^{* 2} U^{11}+\ldots+2 h k a^{*} b^{*} U^{12}\right]$

\begin{tabular}{lcccccc}
\hline & $\mathrm{U}^{11}$ & $\mathrm{U}^{22}$ & $\mathrm{U}^{33}$ & $\mathrm{U}^{23}$ & $\mathrm{U}^{13}$ & $\mathrm{U}^{12}$ \\
\hline $\mathrm{F}(1)$ & $34(1)$ & $43(1)$ & $20(1)$ & $14(1)$ & $8(1)$ & $10(1)$ \\
$\mathrm{F}(2)$ & $39(1)$ & $28(1)$ & $45(1)$ & $14(1)$ & $15(1)$ & $7(1)$ \\
$\mathrm{F}(3)$ & $38(1)$ & $30(1)$ & $37(1)$ & $-6(1)$ & $6(1)$ & $1(1)$ \\
$\mathrm{F}(4)$ & $35(1)$ & $43(1)$ & $16(1)$ & $1(1)$ & $4(1)$ & $7(1)$ \\
$\mathrm{C}(1)$ & $21(1)$ & $34(1)$ & $19(1)$ & $10(1)$ & $7(1)$ & $10(1)$ \\
$\mathrm{C}(2)$ & $23(1)$ & $26(1)$ & $32(1)$ & $10(1)$ & $10(1)$ & $8(1)$ \\
$\mathrm{C}(3)$ & $22(1)$ & $27(1)$ & $28(1)$ & $-1(1)$ & $6(1)$ & $6(1)$ \\
$\mathrm{C}(4)$ & $21(1)$ & $34(1)$ & $17(1)$ & $3(1)$ & $5(1)$ & $9(1)$ \\
$\mathrm{C}(5)$ & $17(1)$ & $30(1)$ & $18(1)$ & $6(1)$ & $6(1)$ & $10(1)$ \\
$\mathrm{O}(1)$ & $36(1)$ & $28(1)$ & $21(1)$ & $1(1)$ & $6(1)$ & $0(1)$ \\
$\mathrm{C}(6)$ & $18(1)$ & $28(1)$ & $22(1)$ & $2(1)$ & $7(1)$ & $4(1)$ \\
$\mathrm{C}(7)$ & $23(1)$ & $35(1)$ & $16(1)$ & $4(1)$ & $5(1)$ & $6(1)$ \\
$\mathrm{C}(8)$ & $24(1)$ & $33(1)$ & $23(1)$ & $10(1)$ & $10(1)$ & $7(1)$ \\
$\mathrm{C}(9)$ & $21(1)$ & $30(1)$ & $23(1)$ & $6(1)$ & $8(1)$ & $5(1)$ \\
$\mathrm{C}(10)$ & $16(1)$ & $29(1)$ & $20(1)$ & $4(1)$ & $7(1)$ & $6(1)$ \\
$\mathrm{C}(11)$ & $38(1)$ & $32(1)$ & $22(1)$ & $-3(1)$ & $3(1)$ & $2(1)$ \\
& & & & & & \\
\hline
\end{tabular}


Table S21. Hydrogen coordinates ( $\left.\times 10^{4}\right)$ and isotropic displacement parameters $\left(\AA^{2} \times 10^{3}\right)$ for complex $\mathbf{2 b} \cdot \mathbf{5 a}$.

\begin{tabular}{lrrrr}
\hline & $x$ & $y$ & $z$ & $U(e q)$ \\
& & & & \\
$H(7)$ & 3422 & 9095 & 708 & 30 \\
$H(8)$ & 4603 & 11927 & 1534 & 31 \\
$H(9)$ & 5811 & 13043 & 4298 & 29 \\
$H(11 A)$ & 1539 & 6596 & 440 & 49 \\
$H(11 B)$ & 2375 & 4884 & 954 & 49 \\
$H(11 C)$ & 3801 & 6250 & 484 & 49 \\
& & & & \\
\hline
\end{tabular}




\subsection{Crystal data and structure for complex 3b·5a (CCDC number: 2060325)}

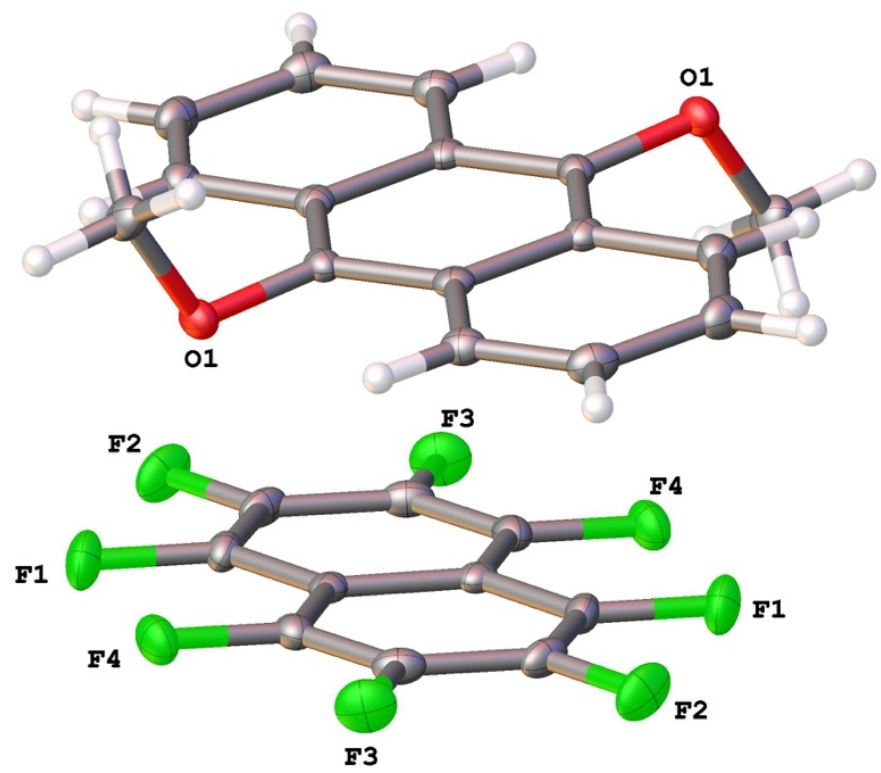

Table S22. Crystal data and structure refinement for complex $\mathbf{3 b} \cdot \mathbf{5 a}$.

Identification code

Empirical formula

Formula weight

Temperature

Wavelength

Crystal system

Space group

Unit cell dimensions

Volume

Z

Density (calculated)

Absorption coefficient

$\mathrm{F}(000)$

Crystal size

Theta range for data collection

Index ranges

Reflections collected

Independent reflections

Completeness to theta $=25.242^{\circ}$

Absorption correction

Max. and min. transmission

Refinement method

Data / restraints / parameters

Goodness-of-fit on $\mathrm{F}^{2}$

Final $\mathrm{R}$ indices [I $>2 \operatorname{sigma}(\mathrm{I})]$

$\mathrm{R}$ indices (all data)

Extinction coefficient

Largest diff. peak and hole
GL 0324914

$\mathrm{C} 2 \overline{6} \mathrm{H} 14 \mathrm{~F} 8 \mathrm{O} 2$

510.37

$100.0 \mathrm{~K}$

$0.71073 \AA$

Triclinic

P-1

$\mathrm{a}=7.0888(19) \AA$

$\alpha=100.337(9)^{\circ}$.

$\mathrm{b}=8.906(2) \AA$

$\beta=100.532(9)^{\circ}$.

$\mathrm{c}=8.942(2) \AA$

510.0(2) $\AA^{3}$

1

$1.662 \mathrm{Mg} / \mathrm{m}^{3}$

$0.154 \mathrm{~mm}^{-1}$

258

$0.33 \times 0.32 \times 0.31 \mathrm{~mm}^{3}$

2.395 to $26.401^{\circ}$.

$-8<=\mathrm{h}<=8,-11<=\mathrm{k}<=11,-11<=\mathrm{l}<=11$

10822

$2071[\mathrm{R}(\mathrm{int})=0.0240]$

$100.0 \%$

Semi-empirical from equivalents

0.7454 and 0.7084

Full-matrix least-squares on $\mathrm{F}^{2}$

2071 / 0 / 164

1.033

$\mathrm{R} 1=0.0290, \mathrm{wR} 2=0.0765$

$\mathrm{R} 1=0.0336, \mathrm{wR} 2=0.0795$

$\mathrm{n} / \mathrm{a}$

0.251 and -0.233 e. $\AA^{-3}$ 
Table S23. Atomic coordinates ( x 10 $0^{4}$ and equivalent isotropic displacement parameters $\left(\AA^{2} \times 10^{3}\right)$ for complex $\mathbf{2 b} \cdot \mathbf{5 a}$. $\mathrm{U}(\mathrm{eq})$ is defined as one third of the trace of the orthogonalized $\mathrm{U}^{\mathrm{ij}}$ tensor.

\begin{tabular}{lcccc}
\hline & $\mathrm{x}$ & $\mathrm{y}$ & $\mathrm{z}$ & $\mathrm{U}(\mathrm{eq})$ \\
\hline $\mathrm{F}(4)$ & $5322(1)$ & $3232(1)$ & $7571(1)$ & $22(1)$ \\
$\mathrm{F}(1)$ & $6080(1)$ & $8614(1)$ & $5271(1)$ & $24(1)$ \\
$\mathrm{F}(3)$ & $6821(1)$ & $6244(1)$ & $9500(1)$ & $26(1)$ \\
$\mathrm{F}(2)$ & $7234(1)$ & $8890(1)$ & $8378(1)$ & $27(1)$ \\
$\mathrm{O}(1)$ & $898(1)$ & $3019(1)$ & $6883(1)$ & $16(1)$ \\
$\mathrm{C}(11)$ & $398(2)$ & $3997(2)$ & $5960(1)$ & $14(1)$ \\
$\mathrm{C}(12)$ & $-572(2)$ & $3294(2)$ & $4358(1)$ & $14(1)$ \\
$\mathrm{C}(10)$ & $973(2)$ & $5675(2)$ & $6630(1)$ & $14(1)$ \\
$\mathrm{C}(5)$ & $4907(2)$ & $4294(1)$ & $5301(1)$ & $14(1)$ \\
$\mathrm{C}(3)$ & $6280(2)$ & $6040(2)$ & $7939(2)$ & $18(1)$ \\
$\mathrm{C}(6)$ & $1195(2)$ & $8422(2)$ & $6331(2)$ & $17(1)$ \\
$\mathrm{C}(1)$ & $5891(2)$ & $7262(2)$ & $5784(2)$ & $16(1)$ \\
$\mathrm{C}(9)$ & $1967(2)$ & $6407(2)$ & $8263(1)$ & $16(1)$ \\
$\mathrm{C}(8)$ & $2540(2)$ & $8051(2)$ & $8869(2)$ & $19(1)$ \\
$\mathrm{C}(4)$ & $5511(2)$ & $4518(2)$ & $6947(2)$ & $16(1)$ \\
$\mathrm{C}(7)$ & $2153(2)$ & $9071(2)$ & $7890(2)$ & $19(1)$ \\
$\mathrm{C}(2)$ & $6480(2)$ & $7420(2)$ & $7356(2)$ & $18(1)$ \\
$\mathrm{C}(13)$ & $-653(2)$ & $2354(2)$ & $7667(2)$ & $20(1)$ \\
\hline & & & & \\
\hline
\end{tabular}


Table S24. Bond lengths $[\AA]$ and angles $\left[{ }^{\circ}\right]$ for complex $\mathbf{2 b} \cdot \mathbf{5 a}$.

\begin{tabular}{|c|c|}
\hline $\mathrm{F}(4)-\mathrm{C}(4)$ & $1.3422(15)$ \\
\hline $\mathrm{F}(1)-\mathrm{C}(1)$ & $1.3411(15)$ \\
\hline $\mathrm{F}(3)-\mathrm{C}(3)$ & $1.3406(15)$ \\
\hline $\mathrm{F}(2)-\mathrm{C}(2)$ & $1.3386(14)$ \\
\hline $\mathrm{O}(1)-\mathrm{C}(11)$ & $1.3825(15)$ \\
\hline $\mathrm{O}(1)-\mathrm{C}(13)$ & $1.4400(15)$ \\
\hline$C(11)-C(12)$ & $1.4014(17)$ \\
\hline$C(11)-C(10)$ & $1.4025(17)$ \\
\hline$C(12)-C(10) \# 1$ & $1.4378(17)$ \\
\hline$C(12)-C(6) \# 1$ & $1.4324(17)$ \\
\hline $\mathrm{C}(10)-\mathrm{C}(9)$ & $1.4314(17)$ \\
\hline$C(5)-C(5) \# 2$ & $1.434(2)$ \\
\hline $\mathrm{C}(5)-\mathrm{C}(1) \# 2$ & $1.4167(17)$ \\
\hline$C(5)-C(4)$ & $1.4142(18)$ \\
\hline$C(3)-C(4)$ & $1.3600(18)$ \\
\hline$C(3)-C(2)$ & $1.3970(19)$ \\
\hline $\mathrm{C}(6)-\mathrm{H}(6)$ & 0.9500 \\
\hline$C(6)-C(7)$ & $1.3625(19)$ \\
\hline $\mathrm{C}(1)-\mathrm{C}(2)$ & $1.3594(19)$ \\
\hline $\mathrm{C}(9)-\mathrm{H}(9)$ & 0.9500 \\
\hline $\mathrm{C}(9)-\mathrm{C}(8)$ & $1.3655(18)$ \\
\hline $\mathrm{C}(8)-\mathrm{H}(8)$ & 0.9500 \\
\hline$C(8)-C(7)$ & $1.4216(19)$ \\
\hline $\mathrm{C}(7)-\mathrm{H}(7)$ & 0.9500 \\
\hline $\mathrm{C}(13)-\mathrm{H}(13 \mathrm{~A})$ & 0.9800 \\
\hline $\mathrm{C}(13)-\mathrm{H}(13 \mathrm{~B})$ & 0.9800 \\
\hline $\mathrm{C}(13)-\mathrm{H}(13 \mathrm{C})$ & 0.9800 \\
\hline $\mathrm{C}(11)-\mathrm{O}(1)-\mathrm{C}(13)$ & $113.35(9)$ \\
\hline $\mathrm{O}(1)-\mathrm{C}(11)-\mathrm{C}(12)$ & $118.78(11)$ \\
\hline $\mathrm{O}(1)-\mathrm{C}(11)-\mathrm{C}(10)$ & $119.17(11)$ \\
\hline $\mathrm{C}(12)-\mathrm{C}(11)-\mathrm{C}(10)$ & $121.95(11)$ \\
\hline $\mathrm{C}(11)-\mathrm{C}(12)-\mathrm{C}(10) \# 1$ & $119.05(11)$ \\
\hline$C(11)-C(12)-C(6) \# 1$ & $122.34(11)$ \\
\hline
\end{tabular}




\begin{tabular}{|c|c|}
\hline $\mathrm{C}(6) \# 1-\mathrm{C}(12)-\mathrm{C}(10) \# 1$ & $118.61(11)$ \\
\hline $\mathrm{C}(11)-\mathrm{C}(10)-\mathrm{C}(12) \# 1$ & $118.99(11)$ \\
\hline $\mathrm{C}(11)-\mathrm{C}(10)-\mathrm{C}(9)$ & $122.45(11)$ \\
\hline $\mathrm{C}(9)-\mathrm{C}(10)-\mathrm{C}(12) \# 1$ & $118.55(11)$ \\
\hline $\mathrm{C}(1) \# 2-\mathrm{C}(5)-\mathrm{C}(5) \# 2$ & $118.28(14)$ \\
\hline $\mathrm{C}(4)-\mathrm{C}(5)-\mathrm{C}(5) \# 2$ & $118.51(14)$ \\
\hline$C(4)-C(5)-C(1) \# 2$ & $123.21(12)$ \\
\hline $\mathrm{F}(3)-\mathrm{C}(3)-\mathrm{C}(4)$ & $120.73(12)$ \\
\hline $\mathrm{F}(3)-\mathrm{C}(3)-\mathrm{C}(2)$ & $118.57(11)$ \\
\hline$C(4)-C(3)-C(2)$ & $120.68(12)$ \\
\hline $\mathrm{C}(12) \# 1-\mathrm{C}(6)-\mathrm{H}(6)$ & 119.5 \\
\hline$C(7)-C(6)-C(12) \# 1$ & $120.92(12)$ \\
\hline $\mathrm{C}(7)-\mathrm{C}(6)-\mathrm{H}(6)$ & 119.5 \\
\hline $\mathrm{F}(1)-\mathrm{C}(1)-\mathrm{C}(5) \# 2$ & $120.22(11)$ \\
\hline $\mathrm{F}(1)-\mathrm{C}(1)-\mathrm{C}(2)$ & $118.65(11)$ \\
\hline$C(2)-C(1)-C(5) \# 2$ & $121.12(12)$ \\
\hline $\mathrm{C}(10)-\mathrm{C}(9)-\mathrm{H}(9)$ & 119.6 \\
\hline$C(8)-C(9)-C(10)$ & $120.84(12)$ \\
\hline $\mathrm{C}(8)-\mathrm{C}(9)-\mathrm{H}(9)$ & 119.6 \\
\hline $\mathrm{C}(9)-\mathrm{C}(8)-\mathrm{H}(8)$ & 119.7 \\
\hline $\mathrm{C}(9)-\mathrm{C}(8)-\mathrm{C}(7)$ & $120.58(12)$ \\
\hline $\mathrm{C}(7)-\mathrm{C}(8)-\mathrm{H}(8)$ & 119.7 \\
\hline $\mathrm{F}(4)-\mathrm{C}(4)-\mathrm{C}(5)$ & $120.76(11)$ \\
\hline $\mathrm{F}(4)-\mathrm{C}(4)-\mathrm{C}(3)$ & $118.28(11)$ \\
\hline$C(3)-C(4)-C(5)$ & $120.96(12)$ \\
\hline$C(6)-C(7)-C(8)$ & $120.50(12)$ \\
\hline $\mathrm{C}(6)-\mathrm{C}(7)-\mathrm{H}(7)$ & 119.8 \\
\hline $\mathrm{C}(8)-\mathrm{C}(7)-\mathrm{H}(7)$ & 119.8 \\
\hline $\mathrm{F}(2)-\mathrm{C}(2)-\mathrm{C}(3)$ & $118.53(12)$ \\
\hline $\mathrm{F}(2)-\mathrm{C}(2)-\mathrm{C}(1)$ & $121.03(12)$ \\
\hline $\mathrm{C}(1)-\mathrm{C}(2)-\mathrm{C}(3)$ & $120.44(11)$ \\
\hline $\mathrm{O}(1)-\mathrm{C}(13)-\mathrm{H}(13 \mathrm{~A})$ & 109.5 \\
\hline $\mathrm{O}(1)-\mathrm{C}(13)-\mathrm{H}(13 \mathrm{~B})$ & 109.5 \\
\hline $\mathrm{O}(1)-\mathrm{C}(13)-\mathrm{H}(13 \mathrm{C})$ & 109.5 \\
\hline $\mathrm{H}(13 \mathrm{~A})-\mathrm{C}(13)-\mathrm{H}(13 \mathrm{~B})$ & 109.5 \\
\hline $\mathrm{H}(13 \mathrm{~A})-\mathrm{C}(13)-\mathrm{H}(13 \mathrm{C})$ & 109.5 \\
\hline
\end{tabular}


Symmetry transformations used to generate equivalent atoms:

$\# 1-x,-y+1,-z+1 \quad \# 2-x+1,-y+1,-z+1$ 
Table S25. Anisotropic displacement parameters $\left(\AA^{2} \times 10^{3}\right)$ for complex $2 \mathbf{b} \cdot 5 \mathbf{a}$. The anisotropic displacement factor exponent takes the form: $-2 \pi^{2}\left[h^{2} a^{* 2} U^{11}+\ldots+2 h k a^{*} b^{*} U^{12}\right]$

\begin{tabular}{lcccccc}
\hline & $\mathrm{U}^{11}$ & $\mathrm{U}^{22}$ & $\mathrm{U}^{33}$ & $\mathrm{U}^{23}$ & $\mathrm{U}^{13}$ & $\mathrm{U}^{12}$ \\
\hline $\mathrm{F}(4)$ & $25(1)$ & $23(1)$ & $23(1)$ & $12(1)$ & $7(1)$ & $12(1)$ \\
$\mathrm{F}(1)$ & $32(1)$ & $12(1)$ & $28(1)$ & $6(1)$ & $12(1)$ & $6(1)$ \\
$\mathrm{F}(3)$ & $27(1)$ & $34(1)$ & $13(1)$ & $2(1)$ & $1(1)$ & $8(1)$ \\
$\mathrm{F}(2)$ & $28(1)$ & $17(1)$ & $24(1)$ & $-6(1)$ & $8(1)$ & $0(1)$ \\
$\mathrm{O}(1)$ & $16(1)$ & $18(1)$ & $17(1)$ & $8(1)$ & $5(1)$ & $8(1)$ \\
$\mathrm{C}(11)$ & $12(1)$ & $16(1)$ & $16(1)$ & $6(1)$ & $6(1)$ & $6(1)$ \\
$\mathrm{C}(12)$ & $11(1)$ & $15(1)$ & $16(1)$ & $4(1)$ & $6(1)$ & $5(1)$ \\
$\mathrm{C}(10)$ & $10(1)$ & $17(1)$ & $15(1)$ & $4(1)$ & $5(1)$ & $5(1)$ \\
$\mathrm{C}(5)$ & $10(1)$ & $15(1)$ & $18(1)$ & $3(1)$ & $5(1)$ & $4(1)$ \\
$\mathrm{C}(3)$ & $13(1)$ & $26(1)$ & $13(1)$ & $2(1)$ & $3(1)$ & $6(1)$ \\
$\mathrm{C}(6)$ & $16(1)$ & $15(1)$ & $20(1)$ & $4(1)$ & $6(1)$ & $7(1)$ \\
$\mathrm{C}(1)$ & $16(1)$ & $12(1)$ & $23(1)$ & $5(1)$ & $9(1)$ & $4(1)$ \\
$\mathrm{C}(9)$ & $14(1)$ & $20(1)$ & $14(1)$ & $5(1)$ & $5(1)$ & $6(1)$ \\
$\mathrm{C}(8)$ & $16(1)$ & $22(1)$ & $14(1)$ & $-1(1)$ & $4(1)$ & $5(1)$ \\
$\mathrm{C}(4)$ & $13(1)$ & $18(1)$ & $20(1)$ & $7(1)$ & $6(1)$ & $7(1)$ \\
$\mathrm{C}(7)$ & $18(1)$ & $15(1)$ & $22(1)$ & $-1(1)$ & $6(1)$ & $5(1)$ \\
$\mathrm{C}(2)$ & $14(1)$ & $14(1)$ & $21(1)$ & $-4(1)$ & $6(1)$ & $1(1)$ \\
$\mathrm{C}(13)$ & $19(1)$ & $20(1)$ & $21(1)$ & $10(1)$ & $6(1)$ & $4(1)$ \\
& & & & & & \\
\hline
\end{tabular}


Table S26. Hydrogen coordinates ( $\left.\times 10^{4}\right)$ and isotropic displacement parameters $\left(\AA^{2} \times 10^{3}\right)$ for complex $\mathbf{2 b} \cdot \mathbf{5 a}$.

\begin{tabular}{lrrrr}
\hline & $x$ & $y$ & $z$ & $U(e q)$ \\
& & & & \\
$H(6)$ & 936 & 9118 & 5693 & 20 \\
$H(9)$ & 2231 & 5741 & 8933 & 19 \\
$H(8)$ & 3202 & 8517 & 9955 & 22 \\
$\mathrm{H}(7)$ & 2566 & 10214 & 8325 & 23 \\
$\mathrm{H}(13 \mathrm{~A})$ & -1962 & 1730 & 6885 & 30 \\
$\mathrm{H}(13 \mathrm{~B})$ & -810 & 3247 & 8390 & 30 \\
$\mathrm{H}(13 \mathrm{C})$ & -242 & 1633 & 8259 & 30 \\
& & & & \\
\hline
\end{tabular}




\section{Computational Results and Coordinates}

Table S27. Computed electronic energies on the co-crystal structures and their truncated structures using energy decomposition analysis (2nd generation ALMO-EDA). Total interaction energies and energy breakdowns are calculated using B97M-V and revPBE functionals with the def2-svpd basis set. Energies in $\mathrm{kcal} \mathrm{mol}^{-1} . \Delta E_{\text {elec }}=$ electrostatic. $\Delta E_{\text {disp }}=$ dispersion. $\Delta E_{\text {Pauli }}=$ Pauli repulsions. $\Delta E_{\mathrm{pol}}=$ polarization. $\Delta E_{\mathrm{CT}}=$ charge transfer. $\Delta E_{\mathrm{int}}=$ total interaction energies.

\begin{tabular}{|c|c|c|c|c|c|c|}
\hline complex & $\Delta E_{\text {elec }}$ & $\Delta E_{\text {disp }}$ & $\Delta E_{\text {Pauli }}$ & $\Delta E_{\mathrm{pol}}$ & $\Delta E_{\mathrm{CT}}$ & $\Delta E_{\text {int }}$ \\
\hline \multicolumn{7}{|c|}{ Fluorination effects } \\
\hline $2 b \cdot 5 a$ & -18.3 & -18.3 & 24.8 & -1.7 & -0.2 & -13.8 \\
\hline $2 b \cdot C_{10} D_{8}$ & -13.8 & -16.7 & 25.5 & -0.8 & -0.4 & -6.2 \\
\hline \multicolumn{7}{|c|}{ Substituent effects } \\
\hline $2 b \cdot 5 a$ & -18.3 & -18.3 & 24.8 & -1.7 & -0.2 & -13.8 \\
\hline $\mathrm{C}_{10} \mathrm{H}_{8} \cdot 5 \mathrm{a}$ & -15.3 & -15.6 & 22.0 & -1.3 & 0.0 & -10.2 \\
\hline$(\mathrm{MeOH})_{2} \cdot 5 \mathrm{a}$ & -5.3 & -3.9 & 5.7 & -0.5 & -0.2 & -4.2 \\
\hline $2 b \cdot C_{10} D_{8}$ & -13.8 & -16.7 & 25.5 & -0.8 & -0.4 & -6.2 \\
\hline $2 b^{\prime} \cdot 5 a$ & -12.5 & -15.8 & 17.4 & -1.3 & 0.0 & -12.2 \\
\hline $2 b " \cdot 5 a$ & -16.9 & -17.4 & 23.2 & -1.5 & -0.1 & -12.7 \\
\hline $\mathrm{C}_{10} \mathrm{H}_{8} \cdot 5 \mathrm{a}$ & -15.3 & -15.6 & 22.0 & -1.3 & 0.0 & -10.2 \\
\hline $\mathrm{C}_{10} \mathrm{H}_{8} \cdot 5 \mathrm{a}$ & -15.1 & -15.1 & 21.6 & -1.3 & -0.1 & -10.4 \\
\hline $\mathrm{C}_{10} \mathrm{H}_{8} " \cdot 5 \mathrm{a}$ & -11.3 & -13.6 & 15.6 & -1.0 & 0.2 & -10.2 \\
\hline
\end{tabular}

\section{Computational Coordinates :}

\section{Complex $\mathbf{C}_{10} \mathbf{H}_{8} \cdot \mathbf{5 a}$}

$\begin{array}{cccc}\mathrm{F} & 5.10000000 & 5.78200000 & 6.49100000 \\ \mathrm{~F} & 4.49300000 & 3.84800000 & 4.74200000 \\ \mathrm{~F} & 4.61000000 & 4.28600000 & 2.09100000 \\ \mathrm{~F} & 5.30500000 & 6.68300000 & 1.11600000 \\ \mathrm{C} & 5.14700000 & 6.05200000 & 5.17700000 \\ \mathrm{C} & 4.85000000 & 5.05900000 & 4.30600000 \\ \mathrm{C} & 4.90700000 & 5.29000000 & 2.92100000 \\ \mathrm{C} & 5.25300000 & 6.51300000 & 2.44900000 \\ \mathrm{C} & 5.55600000 & 7.58300000 & 3.31800000 \\ \mathrm{~F} & 5.95900000 & 9.14600000 & 1.55700000 \\ \mathrm{~F} & 6.56500000 & 11.08000000 & 3.30600000 \\ \mathrm{~F} & 6.44900000 & 10.64200000 & 5.95700000 \\ \mathrm{~F} & 5.75400000 & 8.24500000 & 6.93200000 \\ \mathrm{C} & 5.91200000 & 8.87600000 & 2.87100000 \\ \mathrm{C} & 6.20800000 & 9.86900000 & 3.74200000 \\ \mathrm{C} & 6.15200000 & 9.63800000 & 5.12700000 \\ \mathrm{C} & 5.80600000 & 8.41500000 & 5.59900000 \\ \mathrm{C} & 5.50200000 & 7.34500000 & 4.73000000 \\ \mathrm{C} & 1.78100000 & 6.49900000 & 2.44800000 \\ \mathrm{C} & 1.96500000 & 7.47500000 & 1.49200000 \\ \mathrm{H} & 1.87400000 & 7.26600000 & 0.57000000 \\ \mathrm{C} & 2.28700000 & 8.78500000 & 1.89700000\end{array}$




$\begin{array}{llll}\mathrm{H} & 2.41500000 & 9.45300000 & 1.23400000 \\ \mathrm{C} & 2.42200000 & 9.12300000 & 3.21200000 \\ \mathrm{H} & 2.64600000 & 10.01200000 & 3.45900000 \\ \mathrm{C} & 2.22200000 & 8.12600000 & 4.21100000 \\ \mathrm{C} & 2.33800000 & 8.42900000 & 5.60000000 \\ \mathrm{C} & 2.15500000 & 7.45300000 & 6.55600000 \\ \mathrm{H} & 2.24500000 & 7.66200000 & 7.47900000 \\ \mathrm{C} & 1.83200000 & 6.14300000 & 6.15100000 \\ \mathrm{H} & 1.70400000 & 5.47500000 & 6.81400000 \\ \mathrm{C} & 1.69700000 & 5.80500000 & 4.83600000 \\ \mathrm{H} & 1.47300000 & 4.91600000 & 4.58900000 \\ \mathrm{C} & 1.89700000 & 6.80200000 & 3.83700000 \\ \mathrm{H} & 2.57636612 & 9.45090625 & 5.80925871 \\ \mathrm{H} & 1.54263388 & 5.47709375 & 2.23874129\end{array}$

Complex 2b. $\mathbf{C}_{10} \mathbf{H}_{8}$

$\begin{array}{cccc}\mathrm{C} & 5.14700000 & 6.05200000 & 5.17700000 \\ \mathrm{C} & 4.85000000 & 5.05900000 & 4.30600000 \\ \mathrm{C} & 4.90700000 & 5.29000000 & 2.92100000 \\ \mathrm{C} & 5.25300000 & 6.51300000 & 2.44900000 \\ \mathrm{C} & 5.55600000 & 7.58300000 & 3.31800000 \\ \mathrm{C} & 5.91200000 & 8.87600000 & 2.87100000 \\ \mathrm{C} & 6.20800000 & 9.86900000 & 3.74200000 \\ \mathrm{C} & 6.15200000 & 9.63800000 & 5.12700000 \\ \mathrm{C} & 5.80600000 & 8.41500000 & 5.59900000 \\ \mathrm{C} & 5.50200000 & 7.34500000 & 4.73000000 \\ \mathrm{H} & 5.76462589 & 8.27973847 & 6.65960950 \\ \mathrm{H} & 5.10953379 & 5.83676857 & 6.22445965 \\ \mathrm{H} & 4.56401284 & 4.08888669 & 4.65527283 \\ \mathrm{H} & 4.66914849 & 4.48594978 & 2.25629713 \\ \mathrm{H} & 5.29437411 & 6.64826153 & 1.38839050 \\ \mathrm{H} & 5.94946621 & 9.09123143 & 1.82354035 \\ \mathrm{H} & 6.49398716 & 10.83911331 & 3.39272717 \\ \mathrm{H} & 6.38985151 & 10.44205022 & 5.79170287 \\ \mathrm{O} & 1.47800000 & 5.20000000 & 2.18200000 \\ \mathrm{C} & 1.78100000 & 6.49900000 & 2.44800000 \\ \mathrm{C} & 1.96500000 & 7.47500000 & 1.49200000 \\ \mathrm{H} & 1.87400000 & 7.26600000 & 0.57000000 \\ \mathrm{C} & 2.28700000 & 8.78500000 & 1.89700000 \\ \mathrm{H} & 2.41500000 & 9.45300000 & 1.23400000 \\ \mathrm{C} & 2.42200000 & 9.12300000 & 3.21200000 \\ \mathrm{H} & 2.64600000 & 10.0120000 & 3.45900000 \\ \mathrm{C} & 2.22200000 & 8.12600000 & 4.21100000 \\ \mathrm{C} & 1.43500000 & 4.79400000 & 0.81700000 \\ \mathrm{H} & 0.72000000 & 5.27800000 & 0.35400000 \\ \mathrm{H} & 1.26200000 & 3.83100000 & 0.76800000 \\ \mathrm{H} & 2.29500000 & 4.99400000 & 0.39000000 \\ \mathrm{O} & 2.64100000 & 9.72800000 & 5.86600000 \\ \mathrm{C} & 2.33800000 & 8.42900000 & 5.60000000 \\ \mathrm{C} & 2.15500000 & 7.45300000 & 6.55600000 \\ \mathrm{H} & 2.24500000 & 7.66200000 & 7.47900000 \\ \mathrm{C} & 1.83200000 & 6.14300000 & 6.15100000 \\ \mathrm{H} & 1.70400000 & 5.47500000 & 6.81400000 \\ \mathrm{C} & 1.69700000 & 5.80500000 & 4.83600000 \\ \mathrm{H} & 1.47300000 & 4.91600000 & 4.58900000 \\ \mathrm{C} & 1.89700000 & 6.80200000 & 3.83700000 \\ & & & \\ & & & \end{array}$




$\begin{array}{cccc}\mathrm{C} & 2.68400000 & 10.13400000 & 7.23100000 \\ \mathrm{H} & 3.39900000 & 9.65000000 & 7.69400000 \\ \mathrm{H} & 2.85700000 & 11.09700000 & 7.28000000 \\ \mathrm{H} & 1.82500000 & 9.93400000 & 7.65800000\end{array}$

\section{Complex $(\mathbf{M e O H})_{2} \cdot 5 \mathbf{a}$}

\begin{tabular}{cccc}
\hline $\mathrm{C}$ & 2.51900000 & 10.51500000 & 12.78400000 \\
$\mathrm{H}$ & 2.49316209 & 11.57065890 & 12.61132492 \\
$\mathrm{H}$ & 1.85142568 & 10.02617413 & 12.10555045 \\
$\mathrm{O}$ & 3.84700000 & 10.03100000 & 12.57700000 \\
$\mathrm{C}$ & 7.63800000 & 11.00400000 & 5.13300000 \\
$\mathrm{H}$ & 7.66100000 & 10.03700000 & 5.29100000 \\
$\mathrm{H}$ & 7.91300000 & 11.19100000 & 4.21100000 \\
$\mathrm{H}$ & 8.24900000 & 11.45100000 & 5.75500000 \\
$\mathrm{O}$ & 6.31000000 & 11.48700000 & 5.34000000 \\
$\mathrm{C}$ & 6.53800000 & 14.30700000 & 7.47000000 \\
$\mathrm{C}$ & 5.77200000 & 14.91300000 & 6.51900000 \\
$\mathrm{C}$ & 4.49600000 & 15.38700000 & 6.84200000 \\
$\mathrm{C}$ & 4.01000000 & 15.25100000 & 8.10600000 \\
$\mathrm{C}$ & 4.76900000 & 14.62600000 & 9.12200000 \\
$\mathrm{~F}$ & 7.75600000 & 13.86100000 & 7.11700000 \\
$\mathrm{~F}$ & 6.23100000 & 15.06400000 & 5.27600000 \\
$\mathrm{~F}$ & 3.76300000 & 15.98300000 & 5.89700000 \\
$\mathrm{~F}$ & 2.78800000 & 15.73600000 & 8.36700000 \\
$\mathrm{C}$ & 4.30400000 & 14.46100000 & 10.44700000 \\
$\mathrm{C}$ & 5.07100000 & 13.85500000 & 11.39800000 \\
$\mathrm{C}$ & 6.34600000 & 13.38100000 & 11.07500000 \\
$\mathrm{C}$ & 6.83200000 & 13.51700000 & 9.81100000 \\
$\mathrm{C}$ & 6.07400000 & 14.14200000 & 8.79500000 \\
$\mathrm{~F}$ & 3.08600000 & 14.90700000 & 10.80000000 \\
$\mathrm{~F}$ & 4.61200000 & 13.70400000 & 12.64100000 \\
$\mathrm{~F}$ & 7.08000000 & 12.78500000 & 12.01900000 \\
$\mathrm{~F}$ & 8.05400000 & 13.03200000 & 9.55000000 \\
$\mathrm{H}$ & 2.21770920 & 10.31069428 & 13.79017246 \\
$\mathrm{H}$ & 4.23142123 & 10.18743419 & 11.71135073 \\
$\mathrm{H}$ & 5.92557877 & 11.33056581 & 6.20564927
\end{tabular}

\begin{tabular}{cccc}
\multicolumn{5}{c}{ Complex C $\mathbf{1 0} \mathbf{H} \cdot \mathbf{5 a}$} \\
$\mathrm{C}$ & 3.74200000 & 10.83600000 & 10.27900000 \\
$\mathrm{H}$ & 2.84700000 & 11.13900000 & 10.37800000 \\
$\mathrm{C}$ & 4.39500000 & 10.25400000 & 11.34300000 \\
$\mathrm{C}$ & 5.73400000 & 9.81100000 & 11.20700000 \\
$\mathrm{H}$ & 6.18000000 & 9.41800000 & 11.94900000 \\
$\mathrm{C}$ & 6.38700000 & 9.94600000 & 10.01500000 \\
$\mathrm{H}$ & 7.28400000 & 9.64200000 & 9.93800000 \\
$\mathrm{C}$ & 5.74800000 & 10.53500000 & 8.88600000 \\
$\mathrm{C}$ & 6.41500000 & 10.68200000 & 7.63800000 \\
$\mathrm{H}$ & 7.31000000 & 10.37900000 & 7.53900000 \\
$\mathrm{C}$ & 5.76200000 & 11.26400000 & 6.57400000 \\
$\mathrm{C}$ & 4.42300000 & 11.70800000 & 6.71000000 \\
$\mathrm{H}$ & 3.97700000 & 12.10000000 & 5.96800000 \\
$\mathrm{C}$ & 3.77000000 & 11.57200000 & 7.90200000 \\
$\mathrm{H}$ & 2.87300000 & 11.87600000 & 7.97900000 \\
$\mathrm{C}$ & 4.40900000 & 10.98400000 & 9.03100000 \\
$\mathrm{C}$ & 6.53800000 & 14.30700000 & 7.47000000 \\
$\mathrm{C}$ & 5.77200000 & 14.91300000 & 6.51900000
\end{tabular}




$\begin{array}{cccc}\text { C } & 4.49600000 & 15.38700000 & 6.84200000 \\ \text { C } & 4.01000000 & 15.25100000 & 8.10600000 \\ \text { C } & 4.76900000 & 14.62600000 & 9.12200000 \\ \text { F } & 7.75600000 & 13.86100000 & 7.11700000 \\ \text { F } & 6.23100000 & 15.06400000 & 5.27600000 \\ \text { F } & 3.76300000 & 15.98300000 & 5.89700000 \\ \text { F } & 2.78800000 & 15.73600000 & 8.36700000 \\ \text { C } & 4.30400000 & 14.46100000 & 10.44700000 \\ \text { C } & 5.07100000 & 13.85500000 & 11.39800000 \\ \text { C } & 6.34600000 & 13.38100000 & 11.07500000 \\ \text { C } & 6.83200000 & 13.51700000 & 9.81100000 \\ \text { C } & 6.07400000 & 14.14200000 & 8.79500000 \\ \text { F } & 3.08600000 & 14.90700000 & 10.80000000 \\ \text { F } & 4.61200000 & 13.70400000 & 12.64100000 \\ \text { F } & 7.08000000 & 12.78500000 & 12.01900000 \\ \text { F } & 8.05400000 & 13.03200000 & 9.55000000 \\ \text { H } & 3.96653050 & 10.07964106 & 12.30783825 \\ \text { H } & 6.19046950 & 11.43835894 & 5.60916175\end{array}$

\section{Complex $\mathbf{C}_{10} \mathrm{H}_{8} \cdot \mathbf{5 a} \mathbf{a}^{\prime}$}

\begin{tabular}{lrrr}
\hline $\mathrm{C}$ & 4.88000000 & 0.79600000 & 5.78800000 \\
$\mathrm{H}$ & 3.99900000 & 0.82300000 & 5.46300000 \\
$\mathrm{C}$ & 5.53900000 & -0.39800000 & 5.87100000 \\
$\mathrm{C}$ & 6.88100000 & -0.46100000 & 6.34400000 \\
$\mathrm{H}$ & 7.32800000 & -1.28600000 & 6.39300000 \\
$\mathrm{C}$ & 7.51300000 & 0.69000000 & 6.72400000 \\
$\mathrm{H}$ & 8.40000000 & 0.64600000 & 7.03000000 \\
$\mathrm{C}$ & 7.48100000 & 3.14900000 & 7.09300000 \\
$\mathrm{H}$ & 8.36500000 & 3.12600000 & 7.41200000 \\
$\mathrm{C}$ & 6.82800000 & 4.33300000 & 7.05100000 \\
$\mathrm{H}$ & 7.26100000 & 5.11600000 & 7.33600000 \\
$\mathrm{C}$ & 5.49200000 & 4.39500000 & 6.57600000 \\
$\mathrm{C}$ & 4.85100000 & 3.24300000 & 6.14300000 \\
$\mathrm{H}$ & 3.97100000 & 3.28900000 & 5.81600000 \\
$\mathrm{C}$ & 5.52300000 & 2.00200000 & 6.19400000 \\
$\mathrm{C}$ & 6.86800000 & 1.94000000 & 6.66900000 \\
$\mathrm{C}$ & 5.02000000 & 1.37600000 & 2.02800000 \\
$\mathrm{C}$ & 5.59600000 & 0.12400000 & 2.06100000 \\
$\mathrm{C}$ & 6.86900000 & -0.04300000 & 2.57500000 \\
$\mathrm{C}$ & 7.56800000 & 0.99900000 & 3.07500000 \\
$\mathrm{C}$ & 7.70500000 & 3.43700000 & 3.57900000 \\
$\mathrm{C}$ & 7.15500000 & 4.66500000 & 3.53900000 \\
$\mathrm{C}$ & 5.89900000 & 4.85300000 & 2.97700000 \\
$\mathrm{C}$ & 5.19600000 & 3.81300000 & 2.50100000 \\
$\mathrm{C}$ & 5.71600000 & 2.49200000 & 2.52800000 \\
$\mathrm{C}$ & 7.02400000 & 2.29100000 & 3.07500000 \\
$\mathrm{~F}$ & 3.80700000 & 1.51800000 & 1.50900000 \\
$\mathrm{~F}$ & 4.91000000 & -0.92000000 & 1.58200000 \\
$\mathrm{~F}$ & 7.42400000 & -1.26000000 & 2.54300000 \\
$\mathrm{~F}$ & 8.80200000 & 0.80000000 & 3.57700000 \\
$\mathrm{~F}$ & 8.92300000 & 3.29900000 & 4.11800000 \\
$\mathrm{~F}$ & 7.81200000 & 5.72200000 & 4.03000000 \\
$\mathrm{~F}$ & 5.38900000 & 6.10300000 & 2.91300000 \\
$\mathrm{~F}$ & 3.98900000 & 4.05200000 & 1.97800000 \\
$\mathrm{H}$ & 5.05607189 & 5.37208024 & 6.58944969 \\
$\mathrm{H}$ & 5.13141831 & -1.35554317 & 5.62222653
\end{tabular}




\section{NMR spectra}

${ }^{1} \mathrm{H}$ NMR $(500 \mathrm{MHz}$, Chloroform- $d$ )

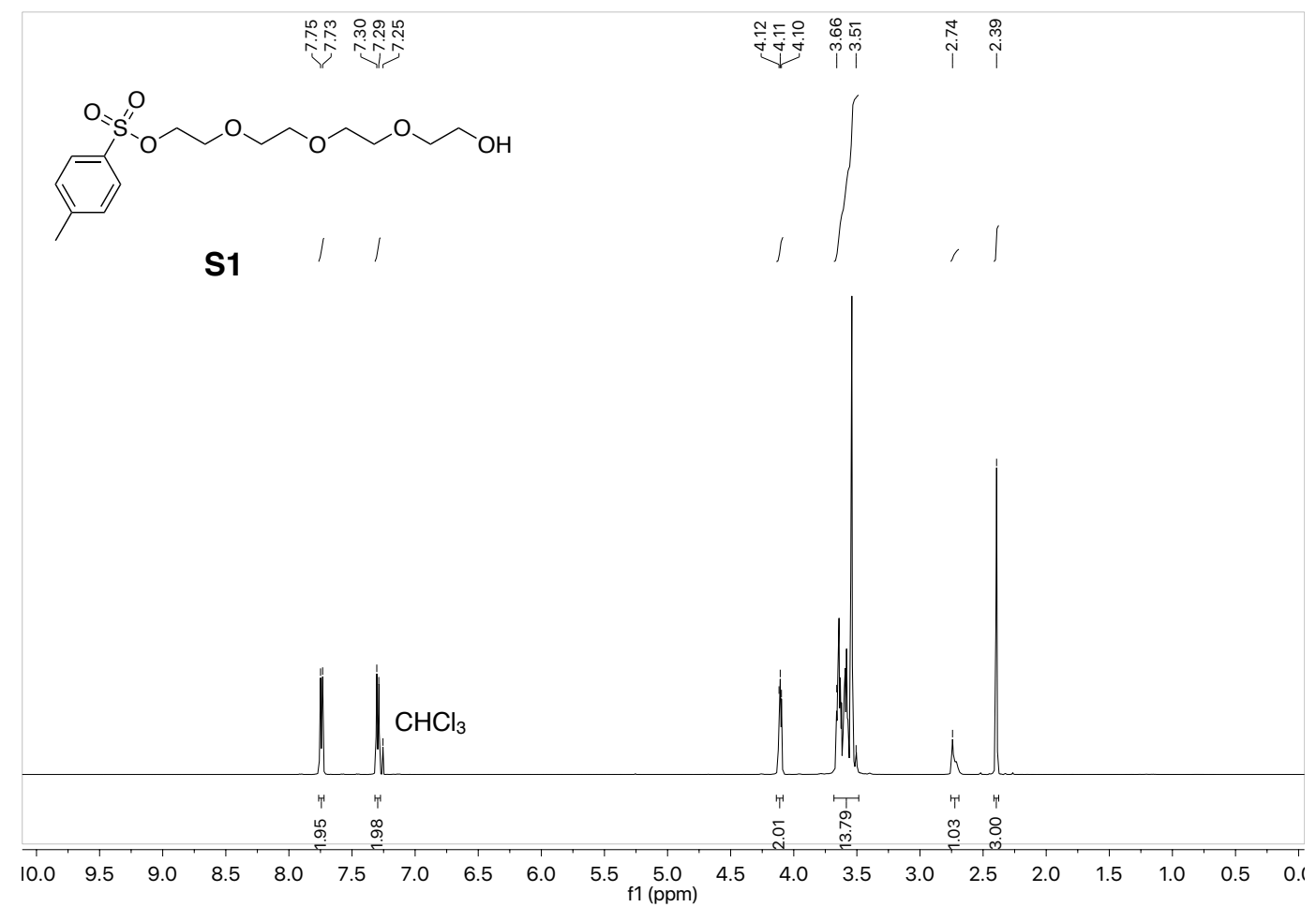

${ }^{13} \mathrm{C}$ NMR (126 MHz, Chloroform- $d$ )

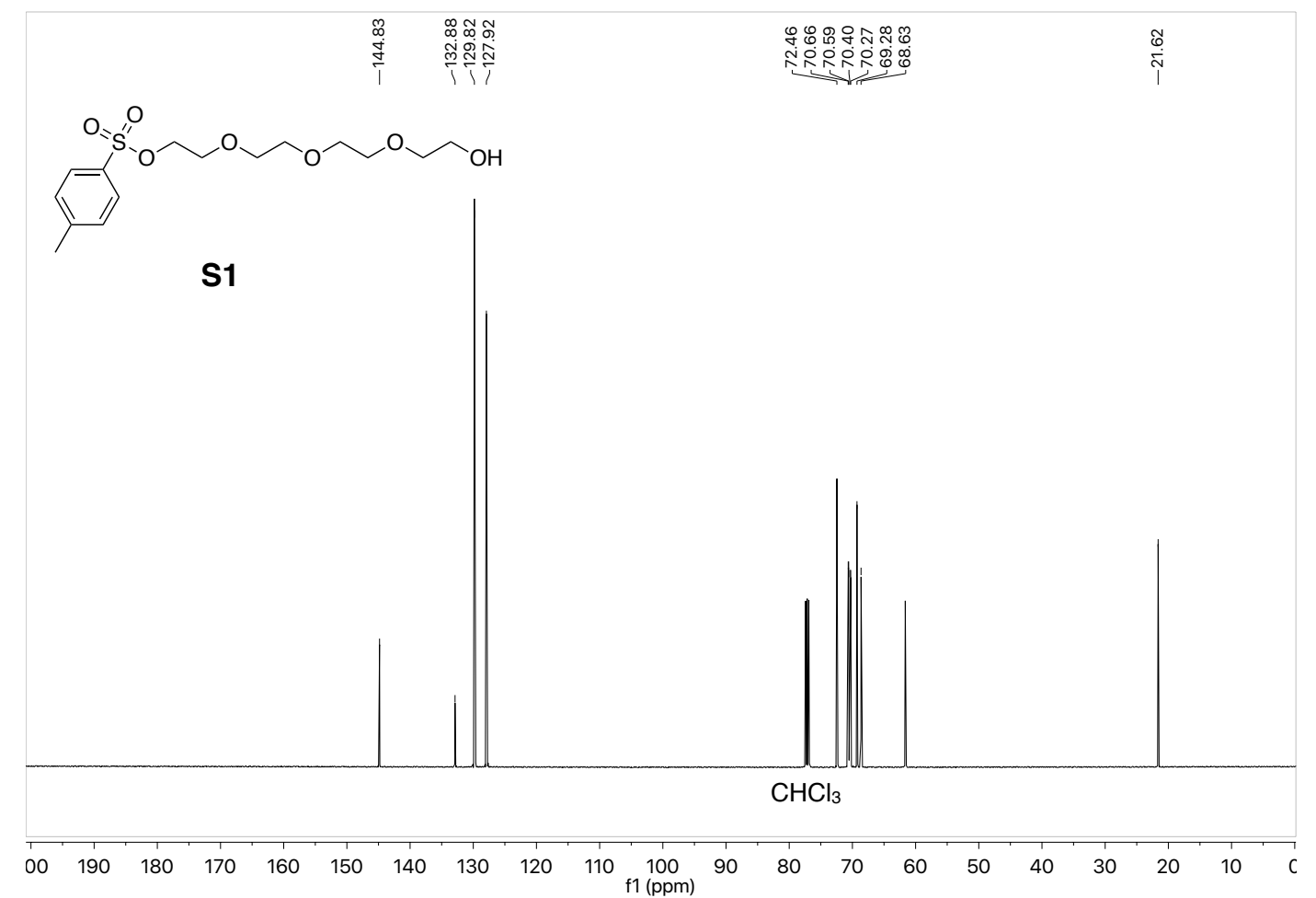


${ }^{1} \mathrm{H}$ NMR (500 MHz, Chloroform- $d$ )

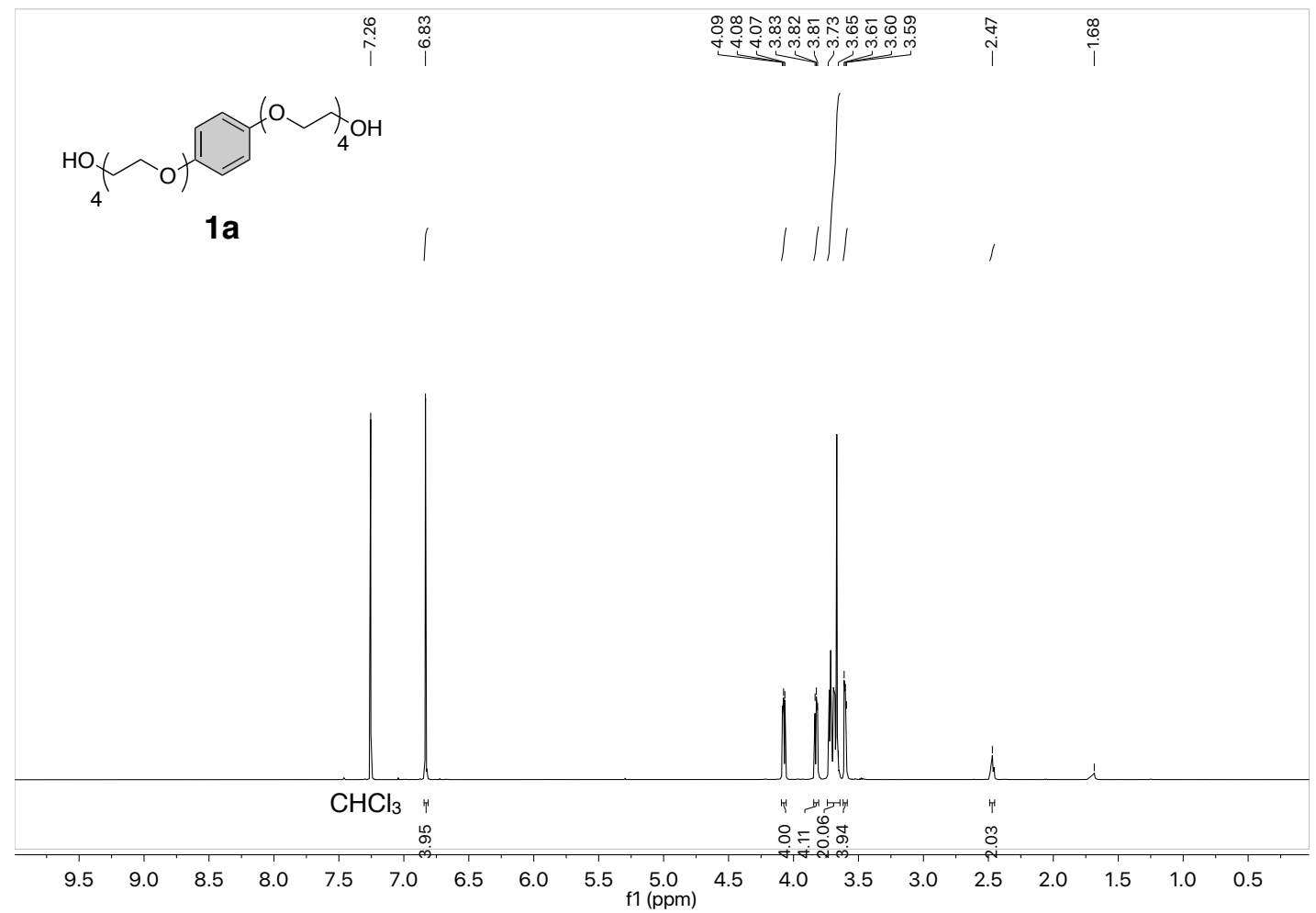

${ }^{13} \mathrm{C}$ NMR (126 MHz, Chloroform- $d$ )

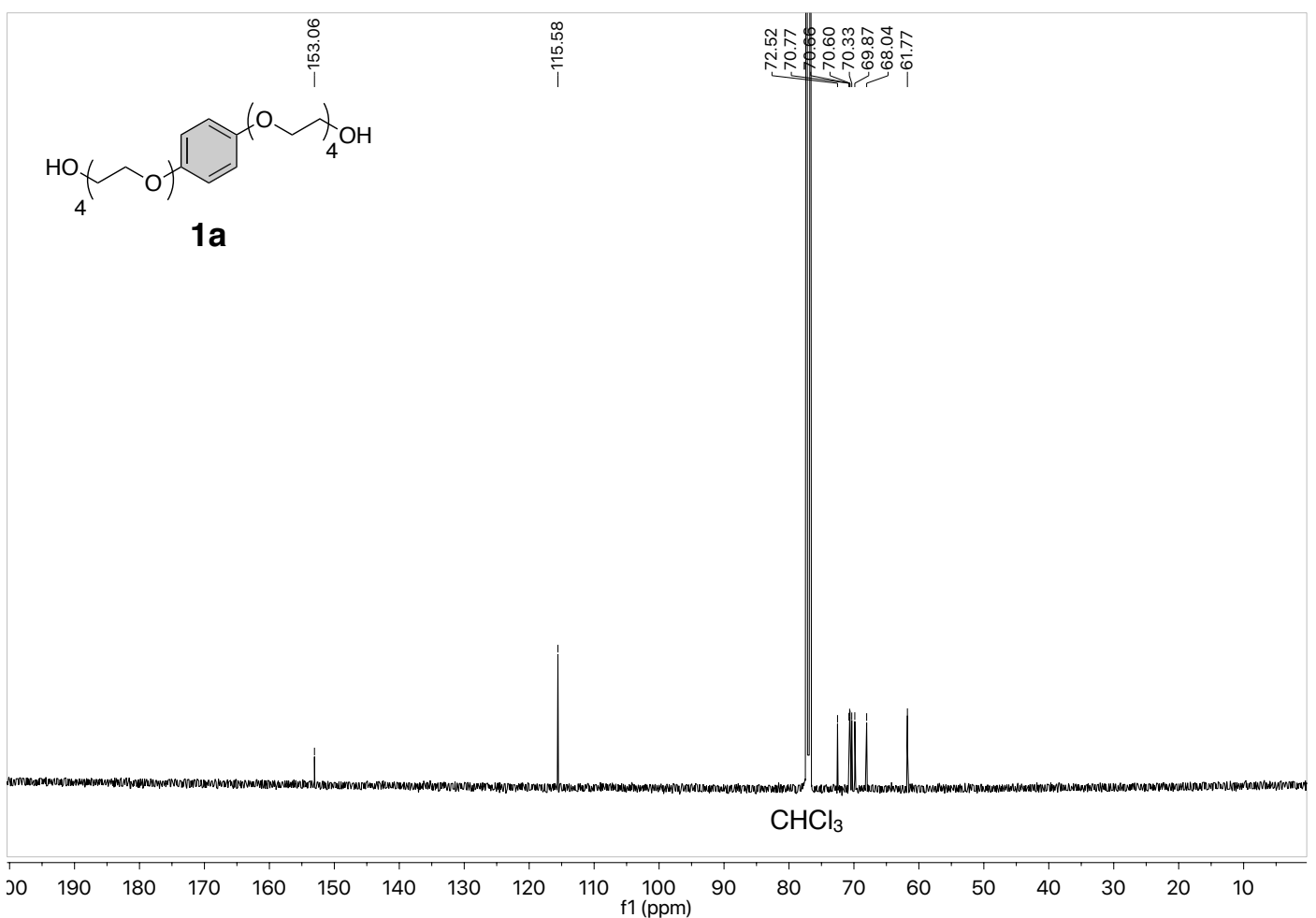


1H NMR (500 MHz, Chloroform-d)
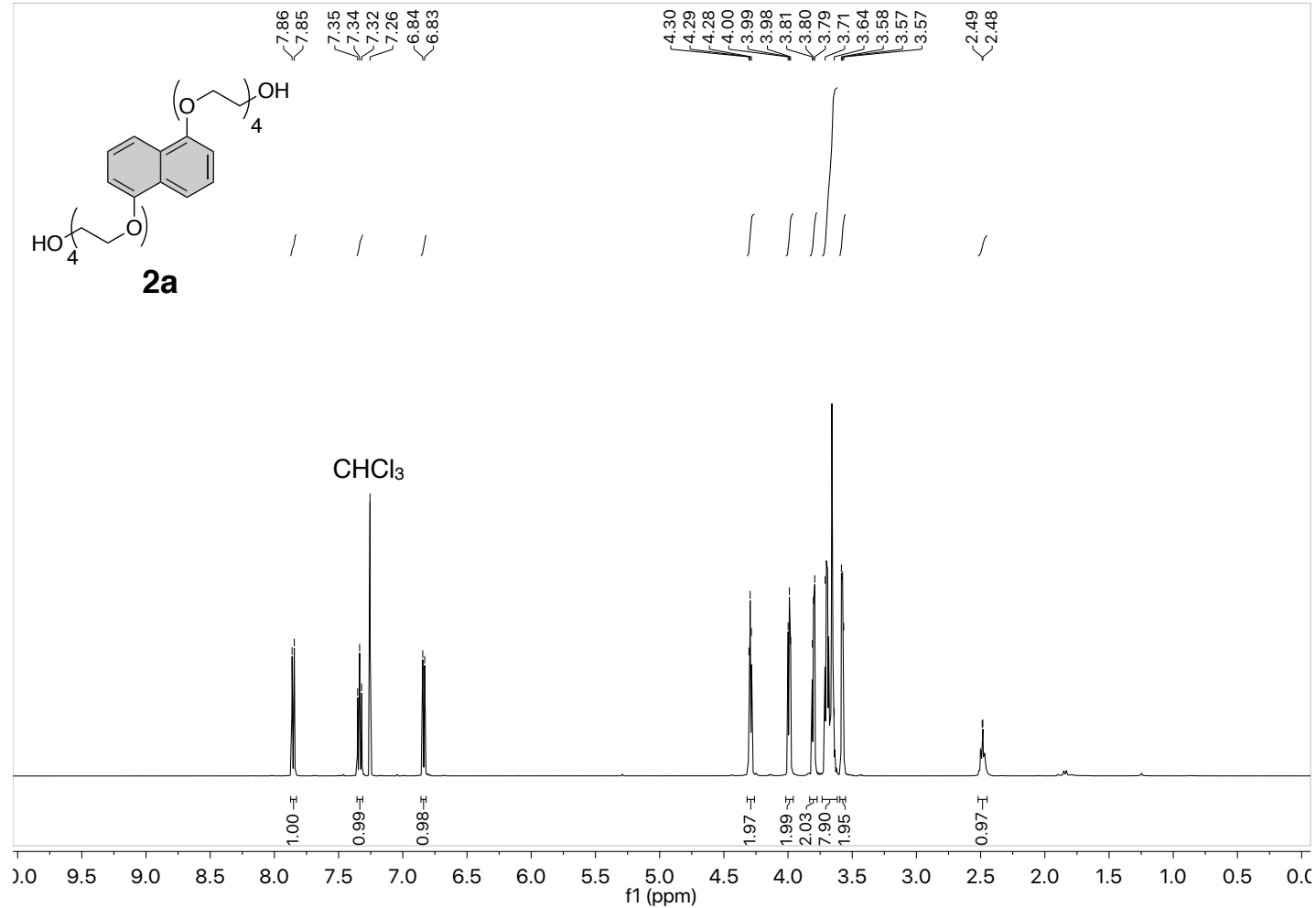

${ }^{13} \mathrm{C}$ NMR (126 MHz, Chloroform-d)

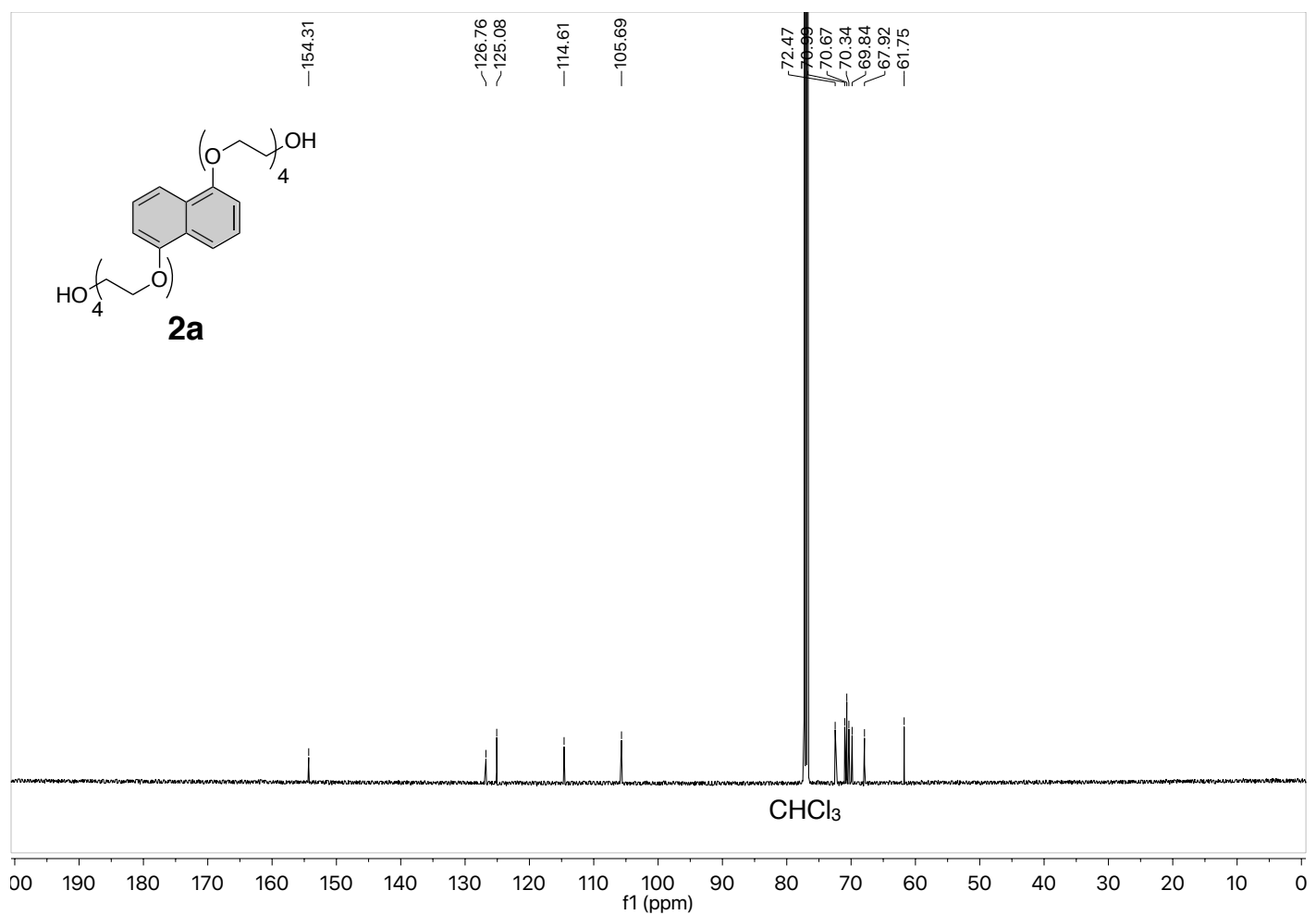


1H NMR (500 MHz, Chloroform-d)

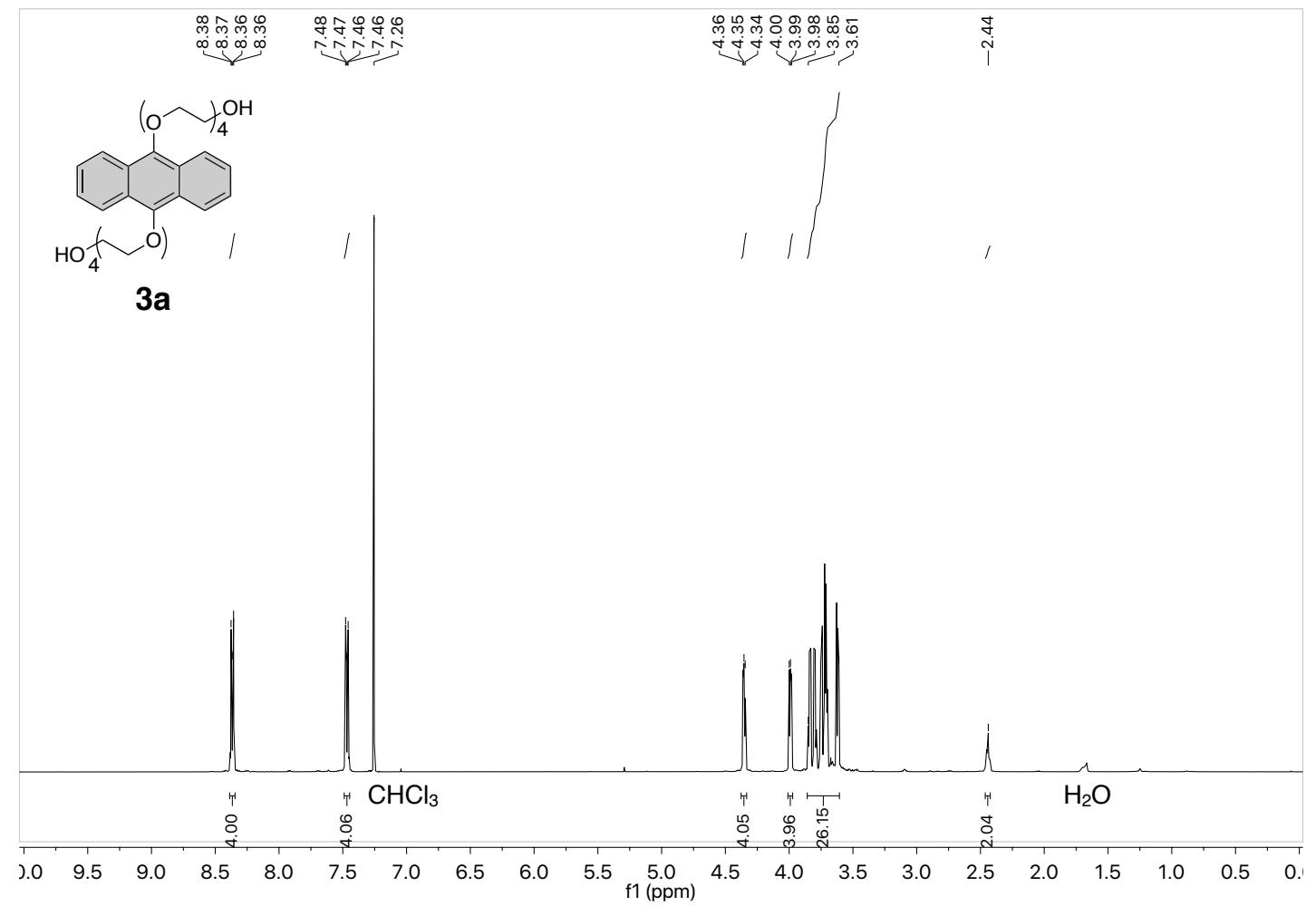

${ }^{13} \mathrm{C}$ NMR (126 MHz, Chloroform- $d$ )

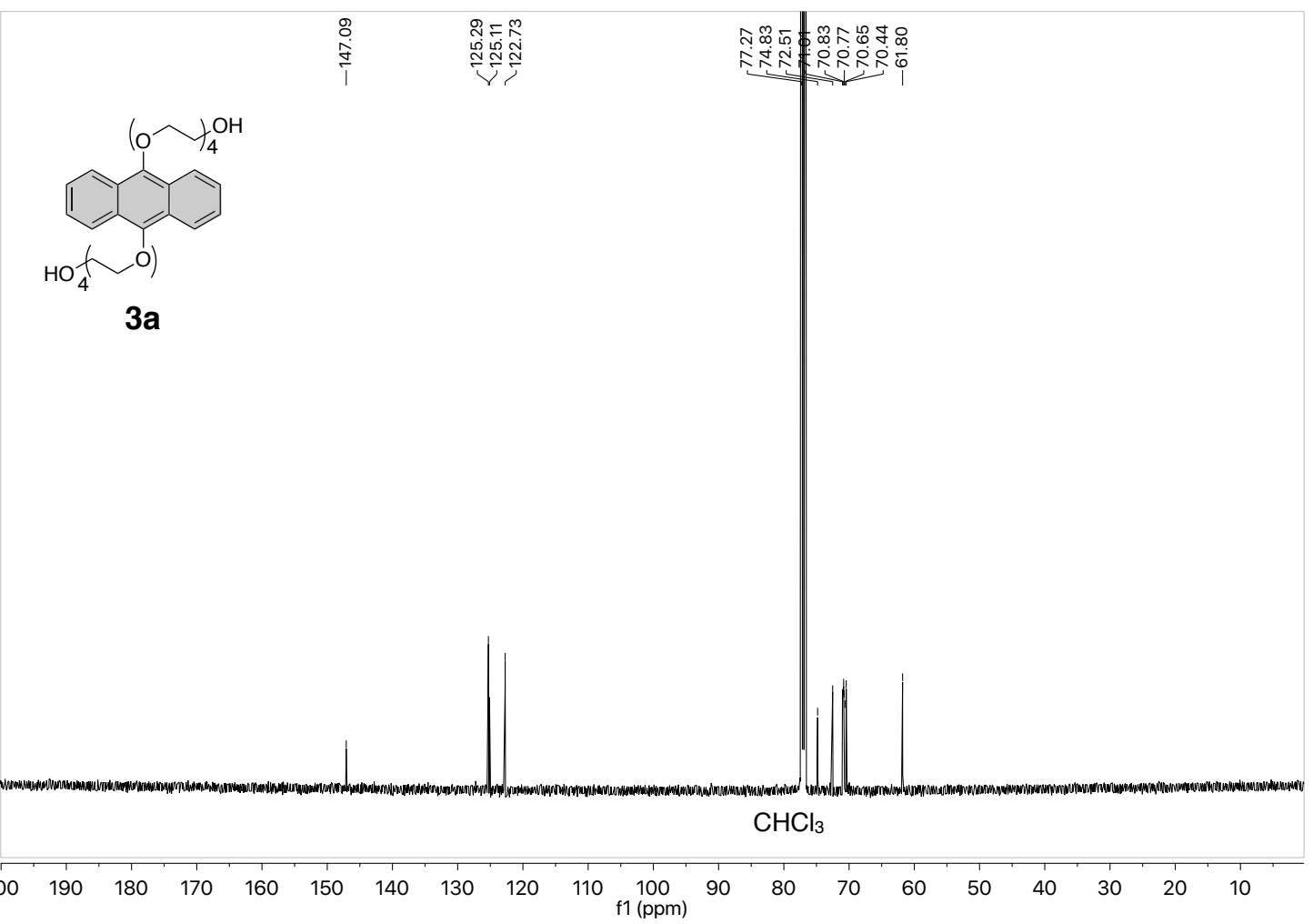


1H NMR (500 MHz, Chloroform-d)

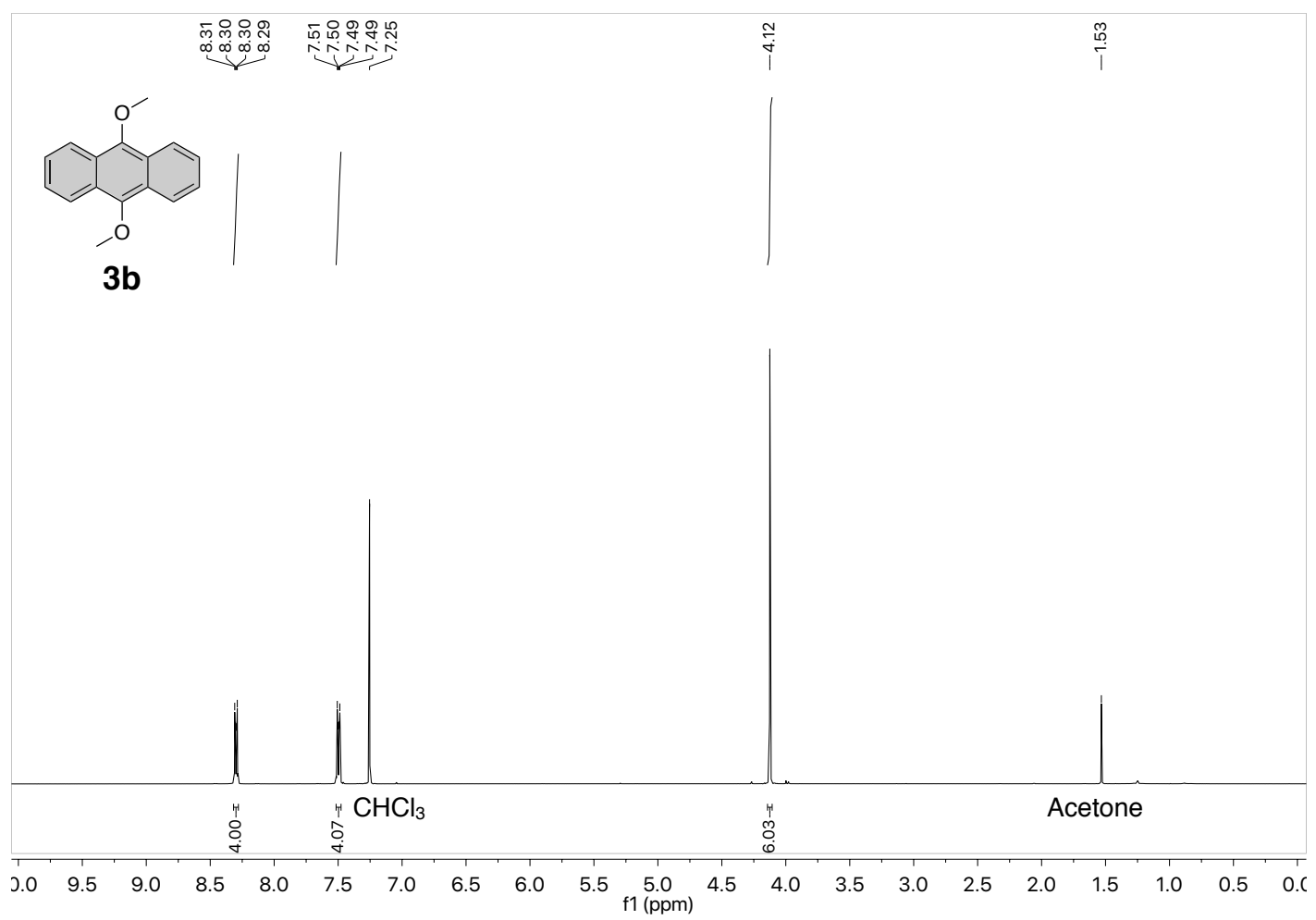

${ }^{13} \mathrm{C}$ NMR (126 MHz, Chloroform- $d$ )

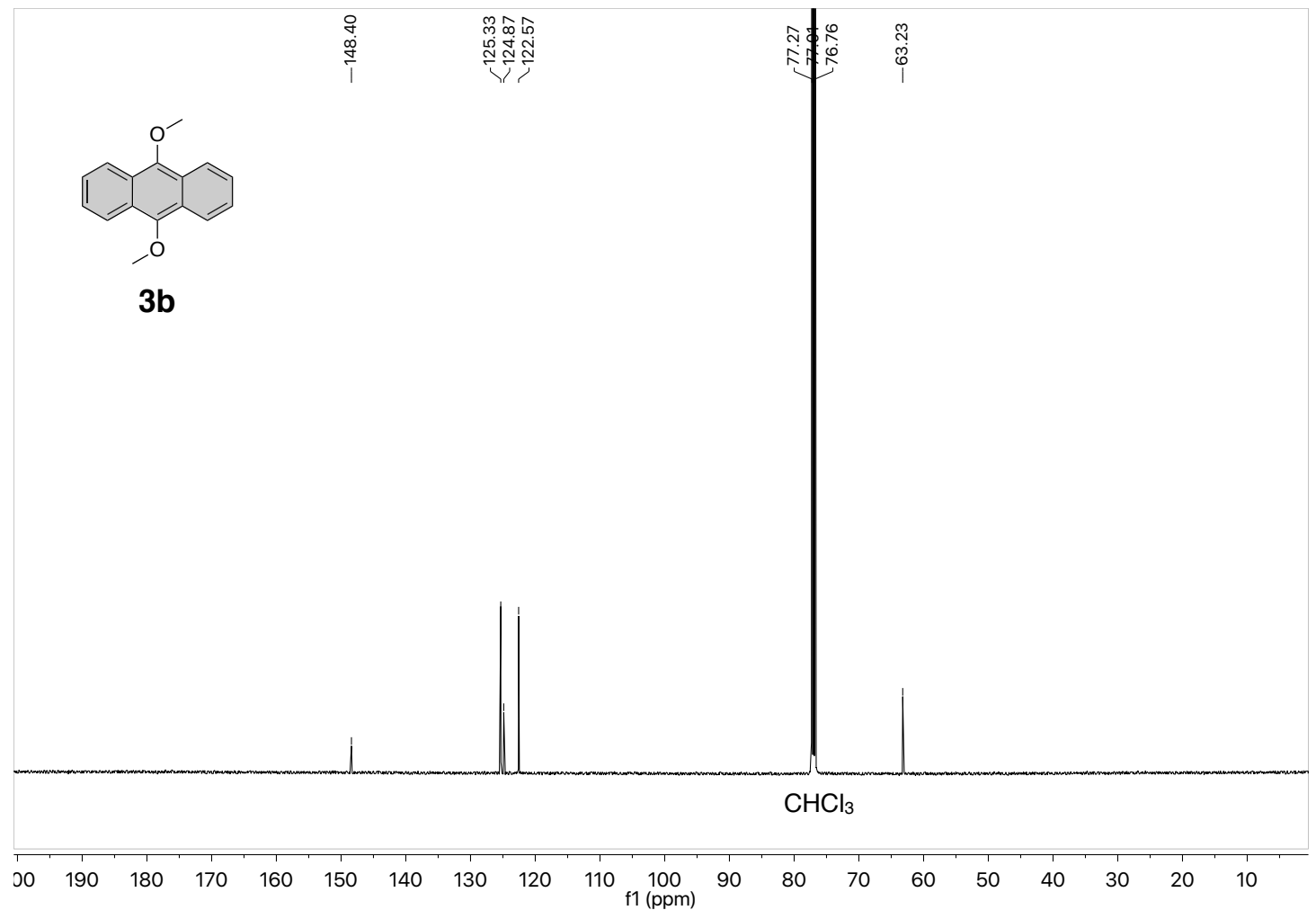


1H NMR (500 MHz, Chloroform-d)

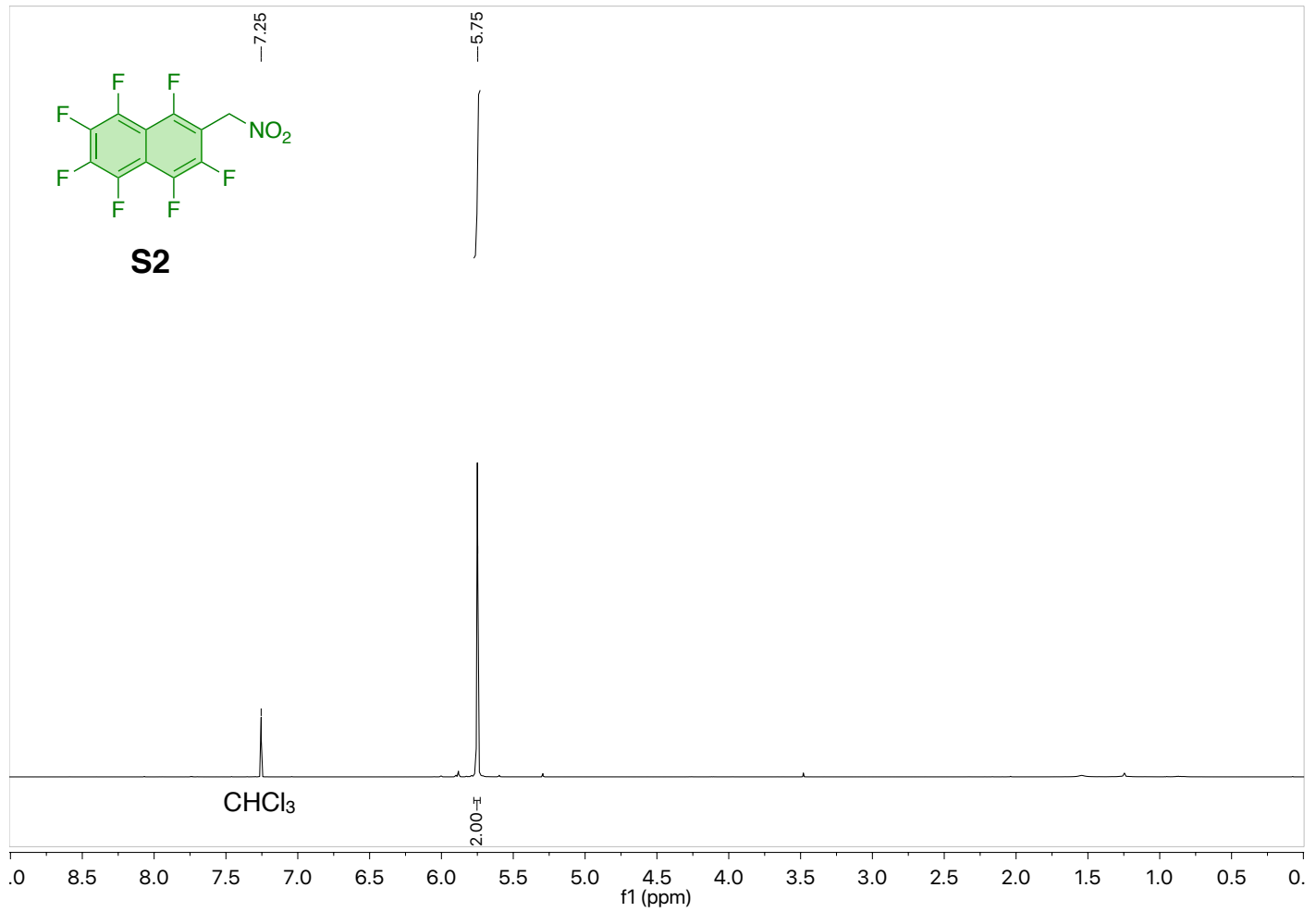

${ }^{13} \mathrm{C}$ NMR (126 MHz, Chloroform- $d$ )

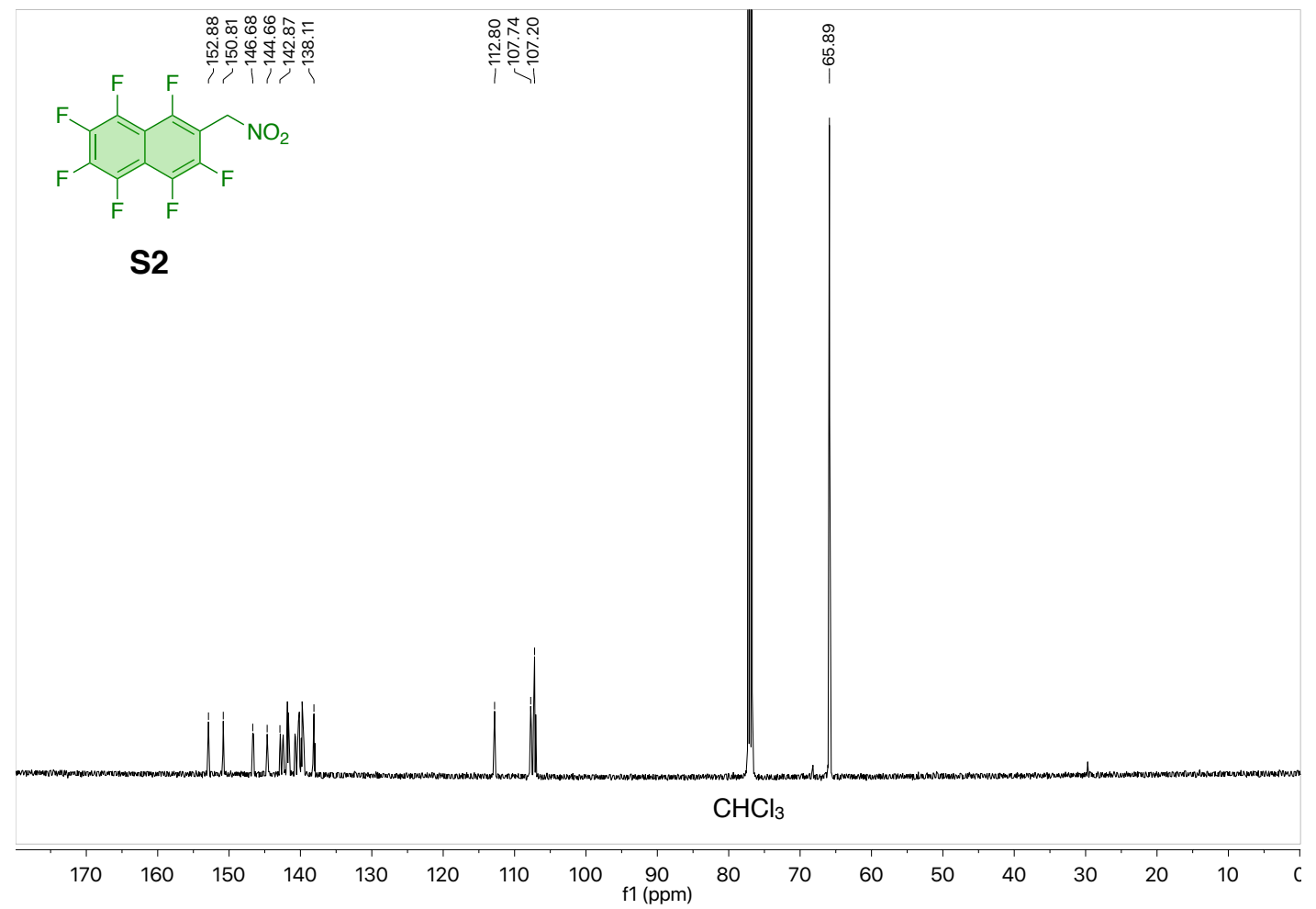


19F NMR (376 MHz, Chloroform- $d$ )

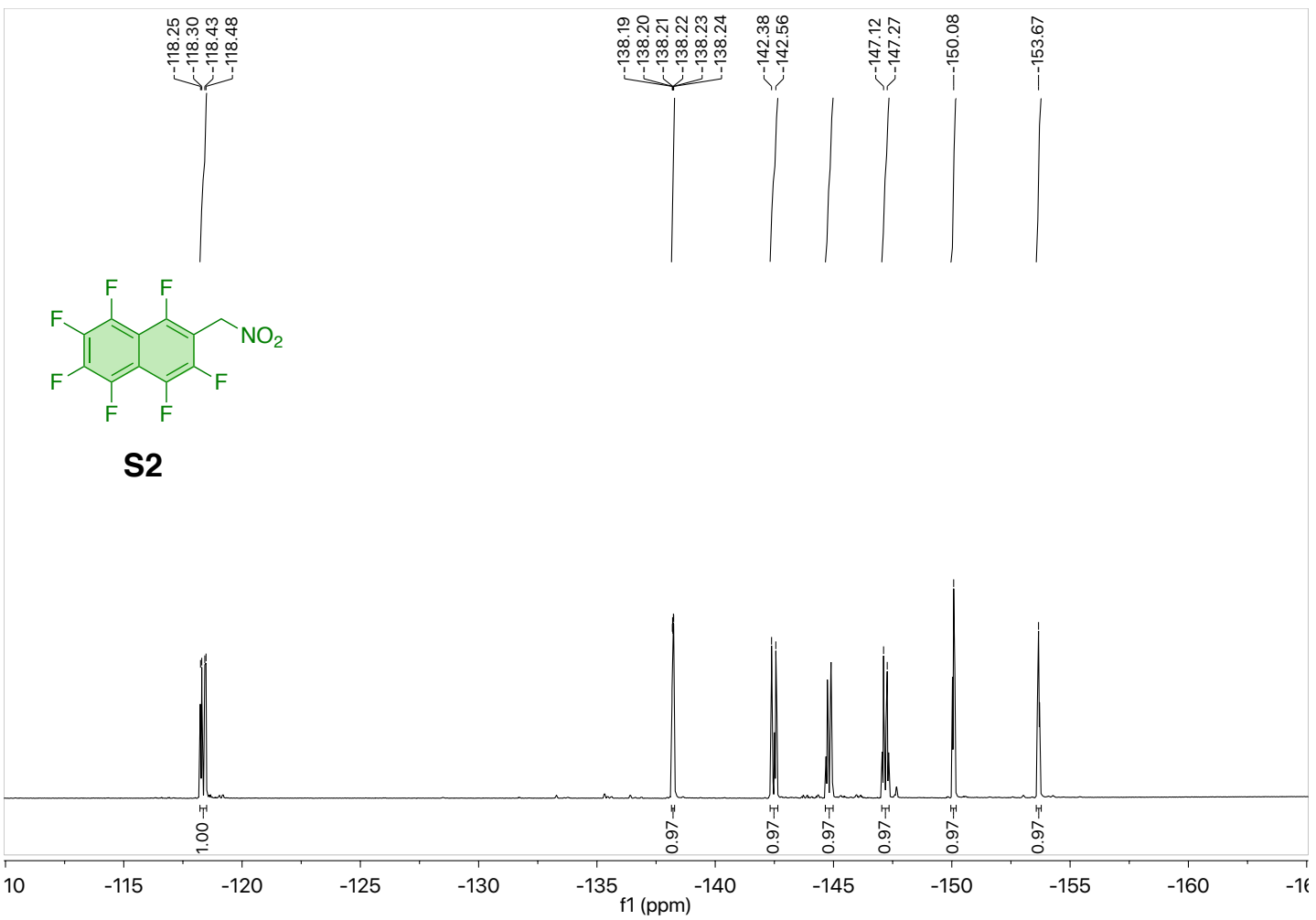

${ }^{1} \mathrm{H}$ NMR (500 MHz, Chloroform- $d$ )

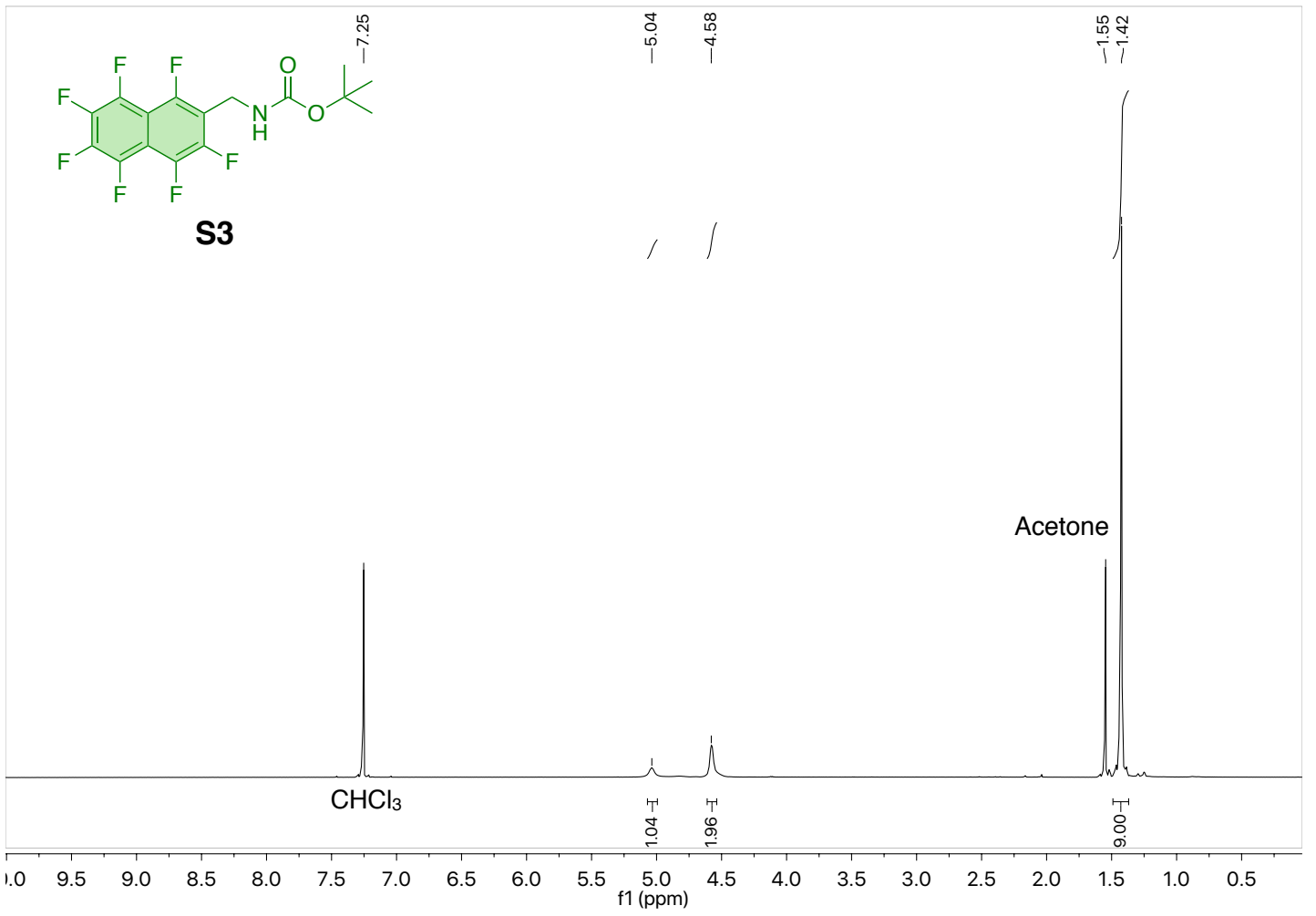


${ }^{13} \mathrm{C}$ NMR (126 MHz, Chloroform- $d$ )

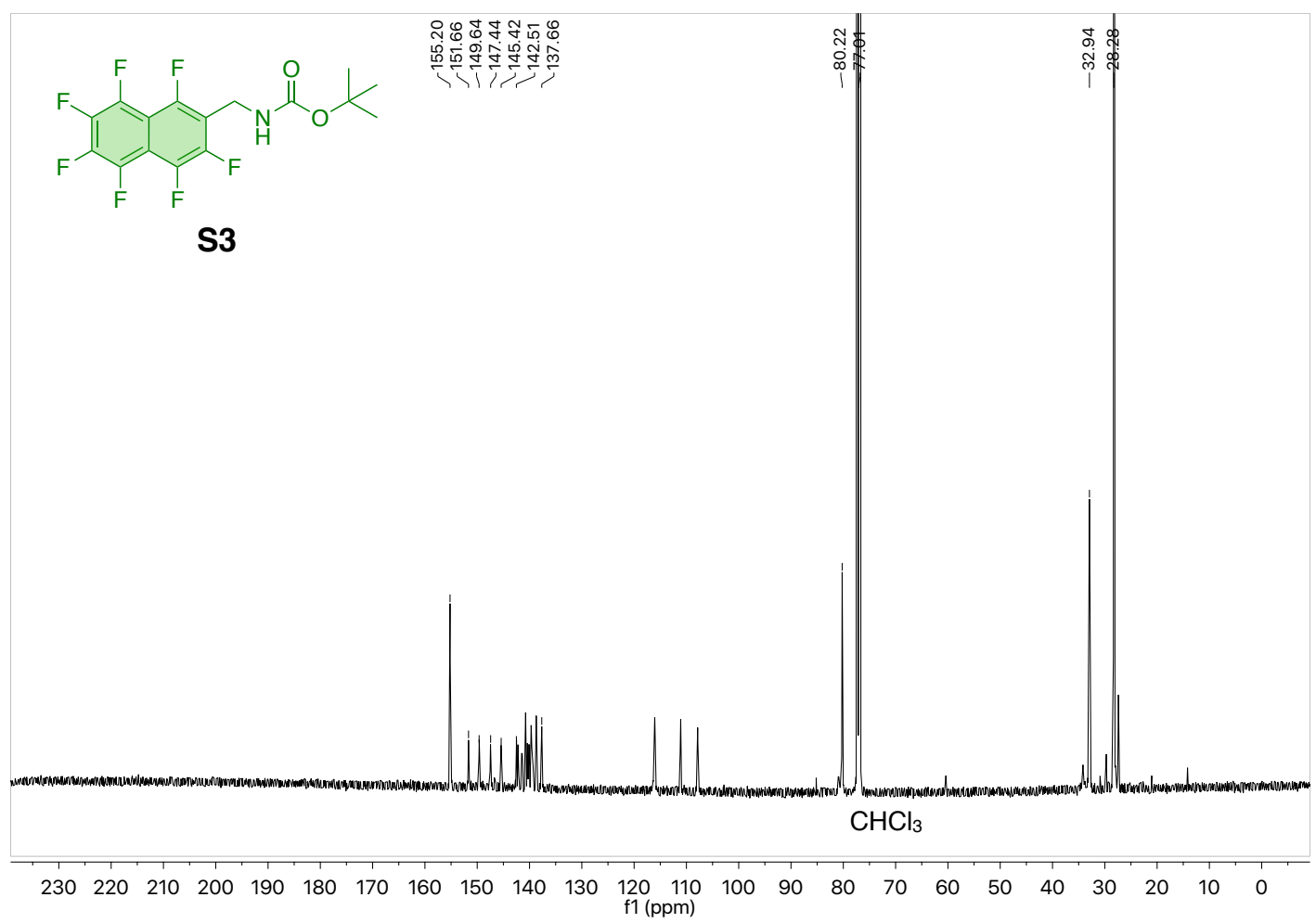

19F NMR (376 MHz, Chloroform-d)
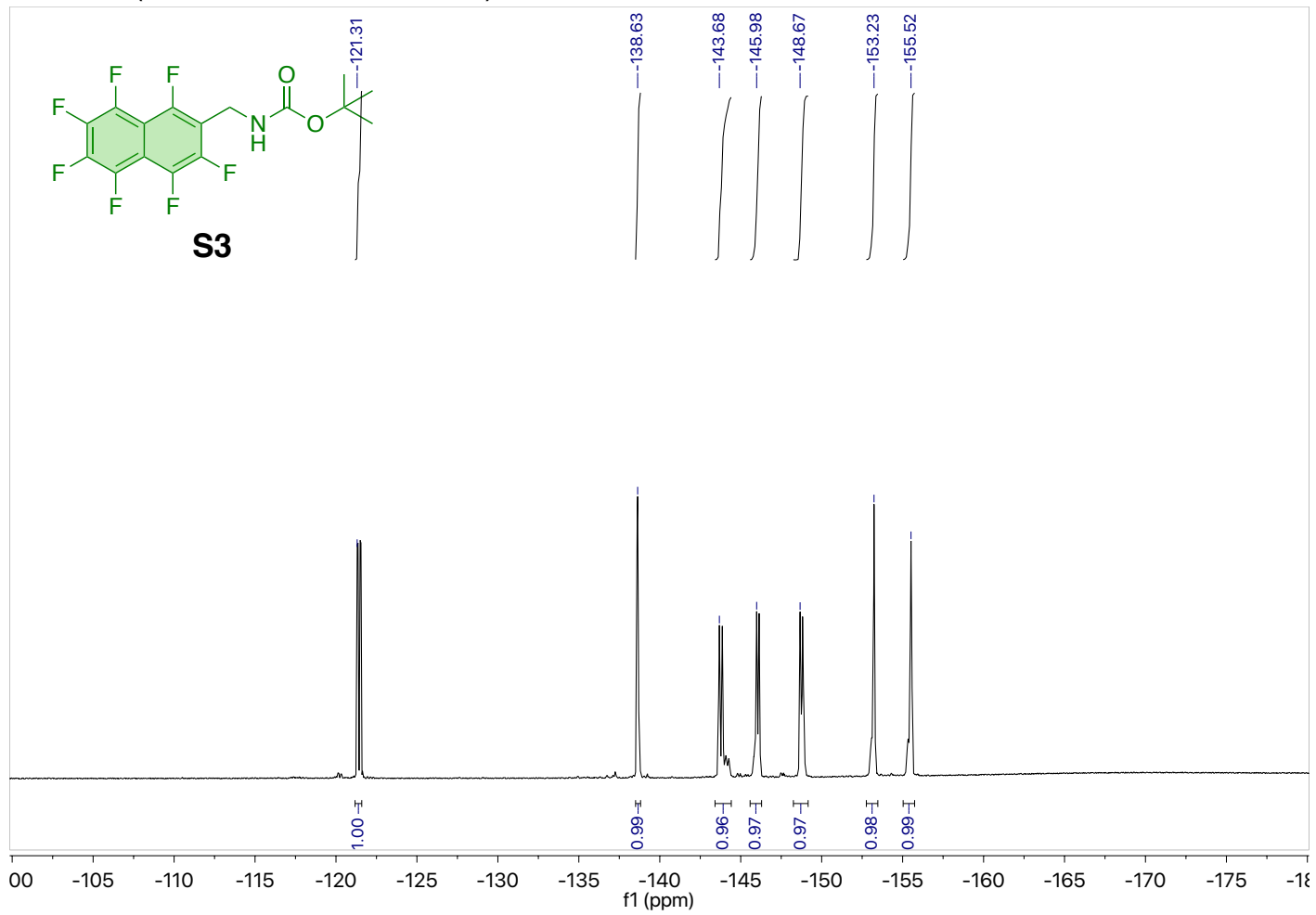
${ }^{1} \mathrm{H}$ NMR $\left(500 \mathrm{MHz}\right.$, Methanol- $\left.d_{4}\right)$

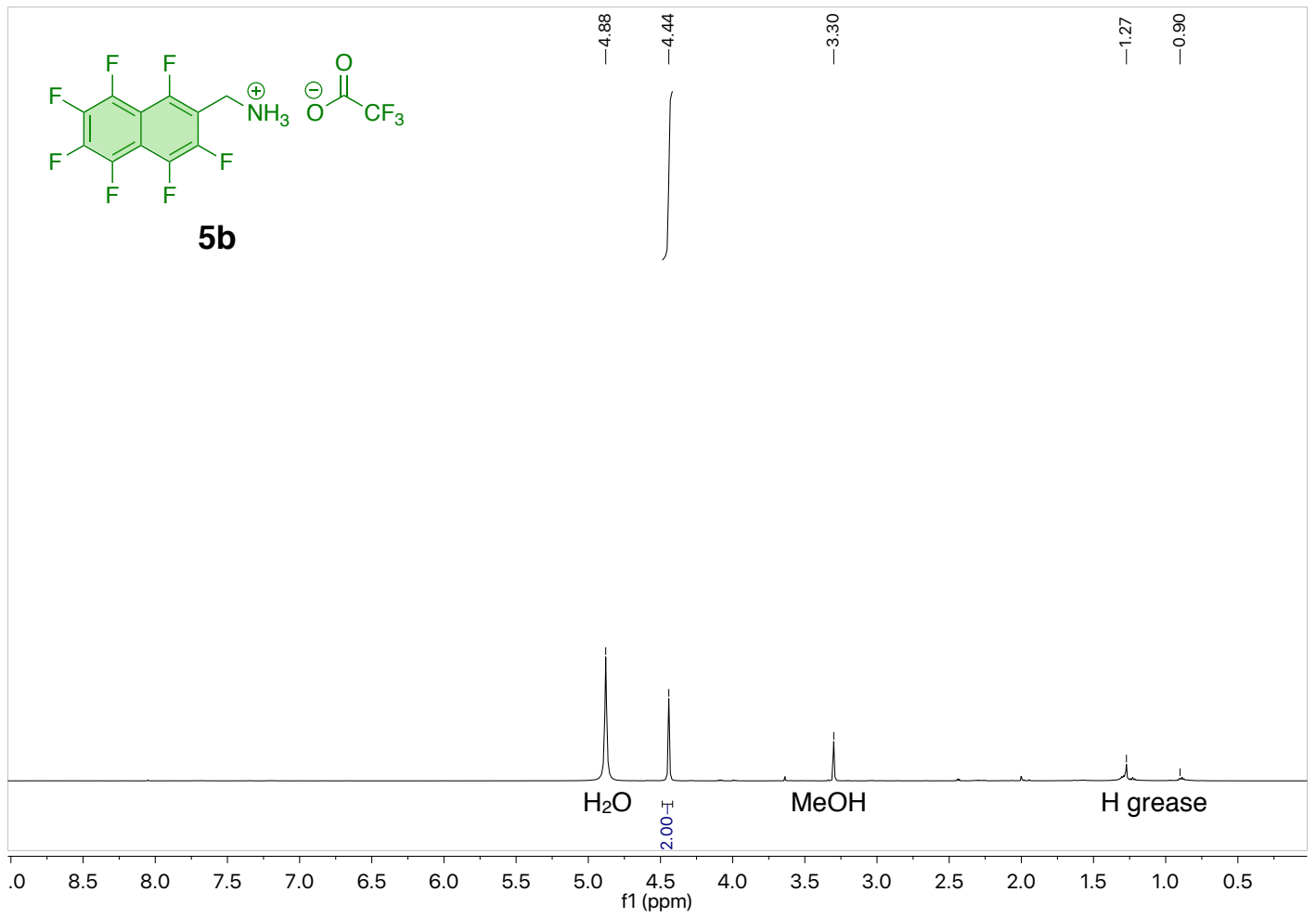

${ }^{13} \mathrm{C}$ NMR $\left(126 \mathrm{MHz}\right.$, Methanol- $\left.d_{4}\right)$

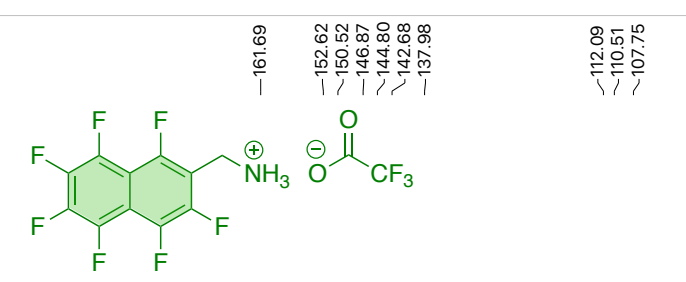

$5 b$

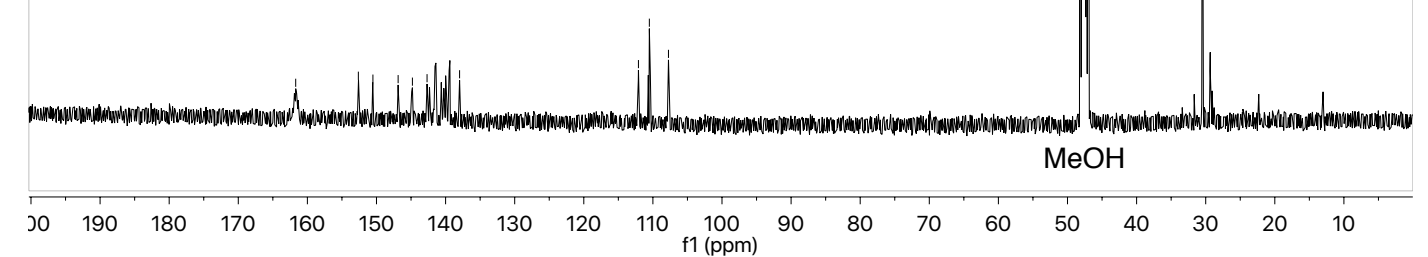


19F NMR (376 MHz, Methanol- $\left.d_{4}\right)$

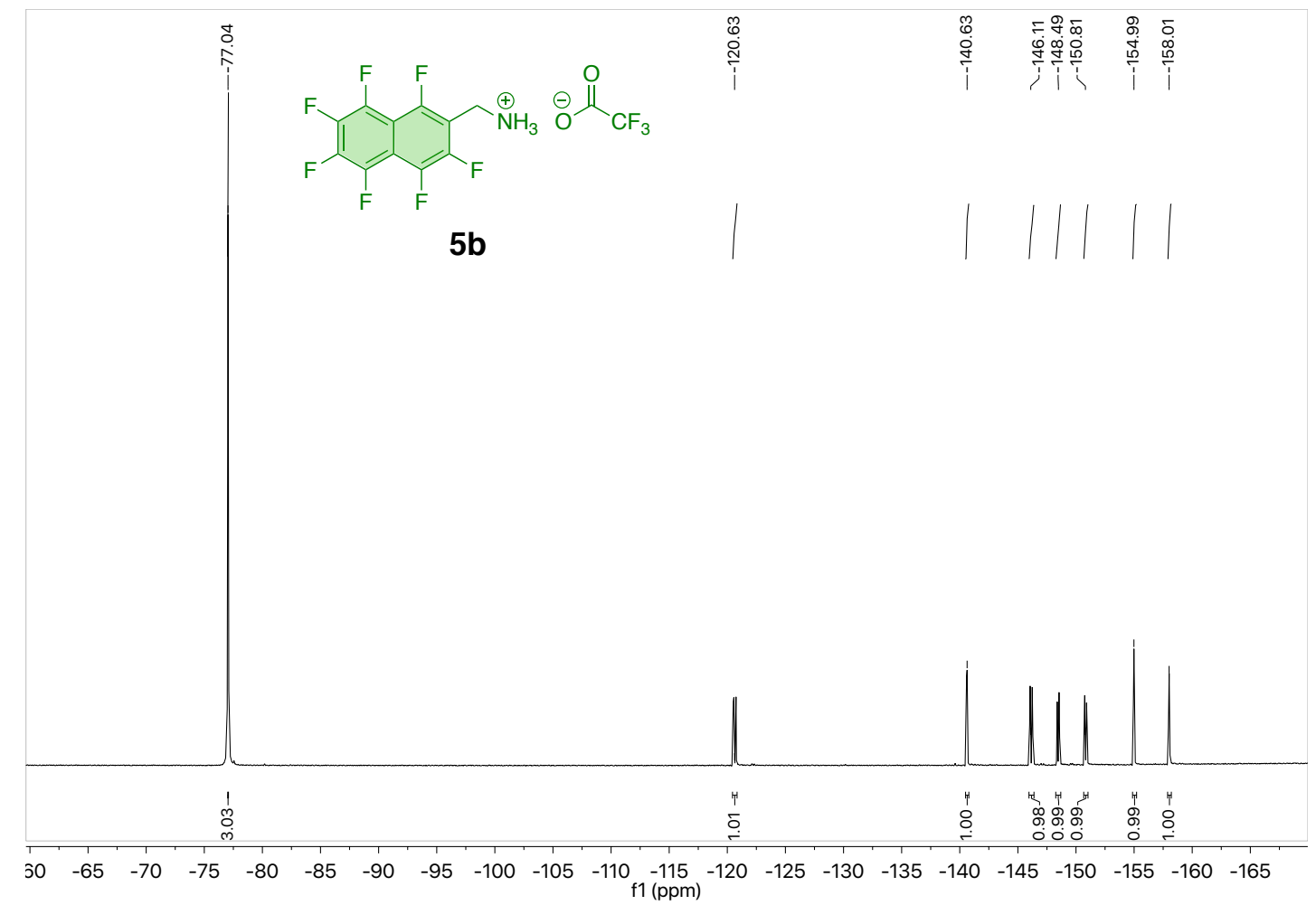

${ }^{1} \mathrm{H}$ NMR $\left(500 \mathrm{MHz}\right.$, Methanol- $\left.d_{4}\right)$

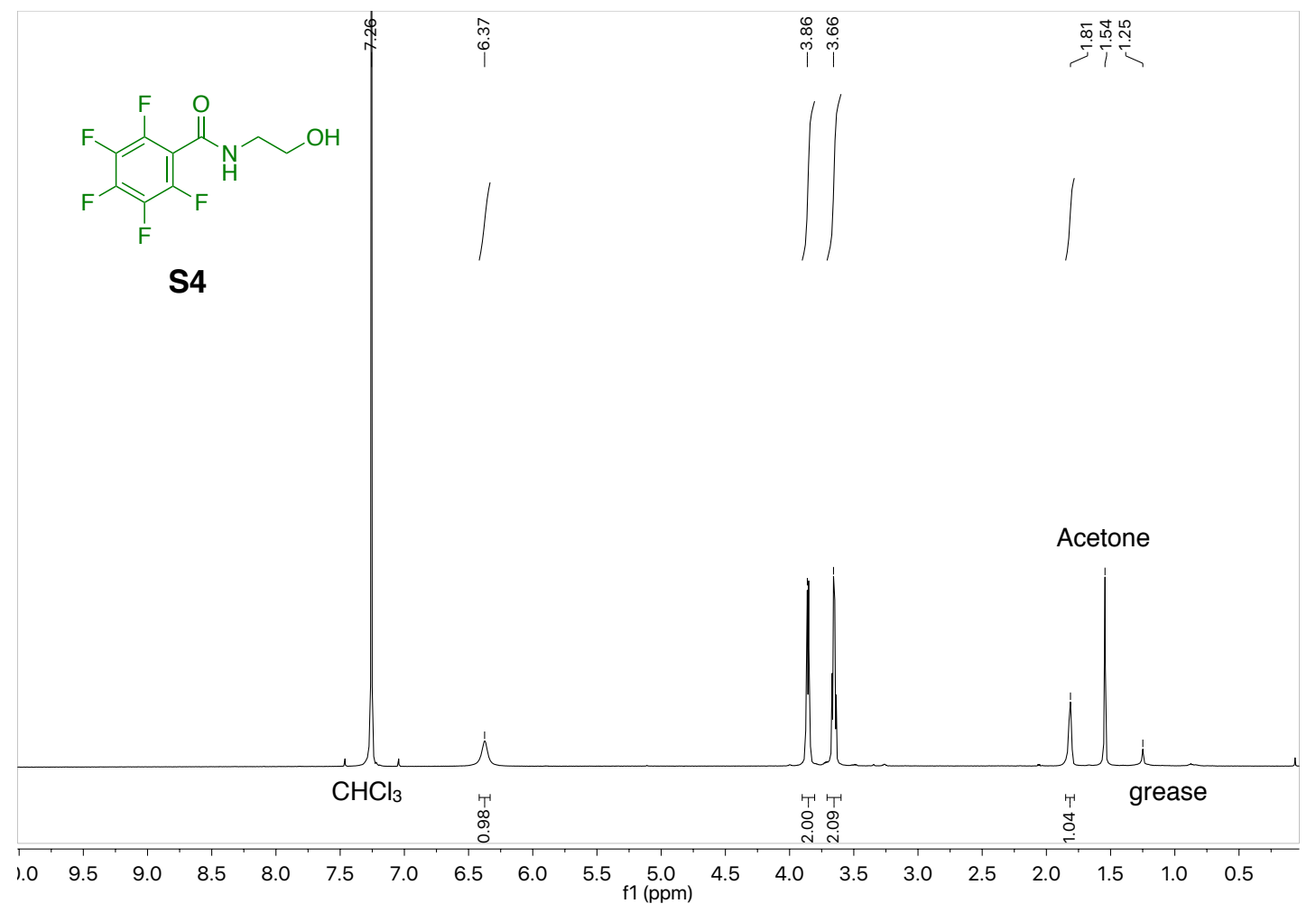


${ }^{13} \mathrm{C}$ NMR (126 MHz, Methanol- $\left.d_{4}\right)$

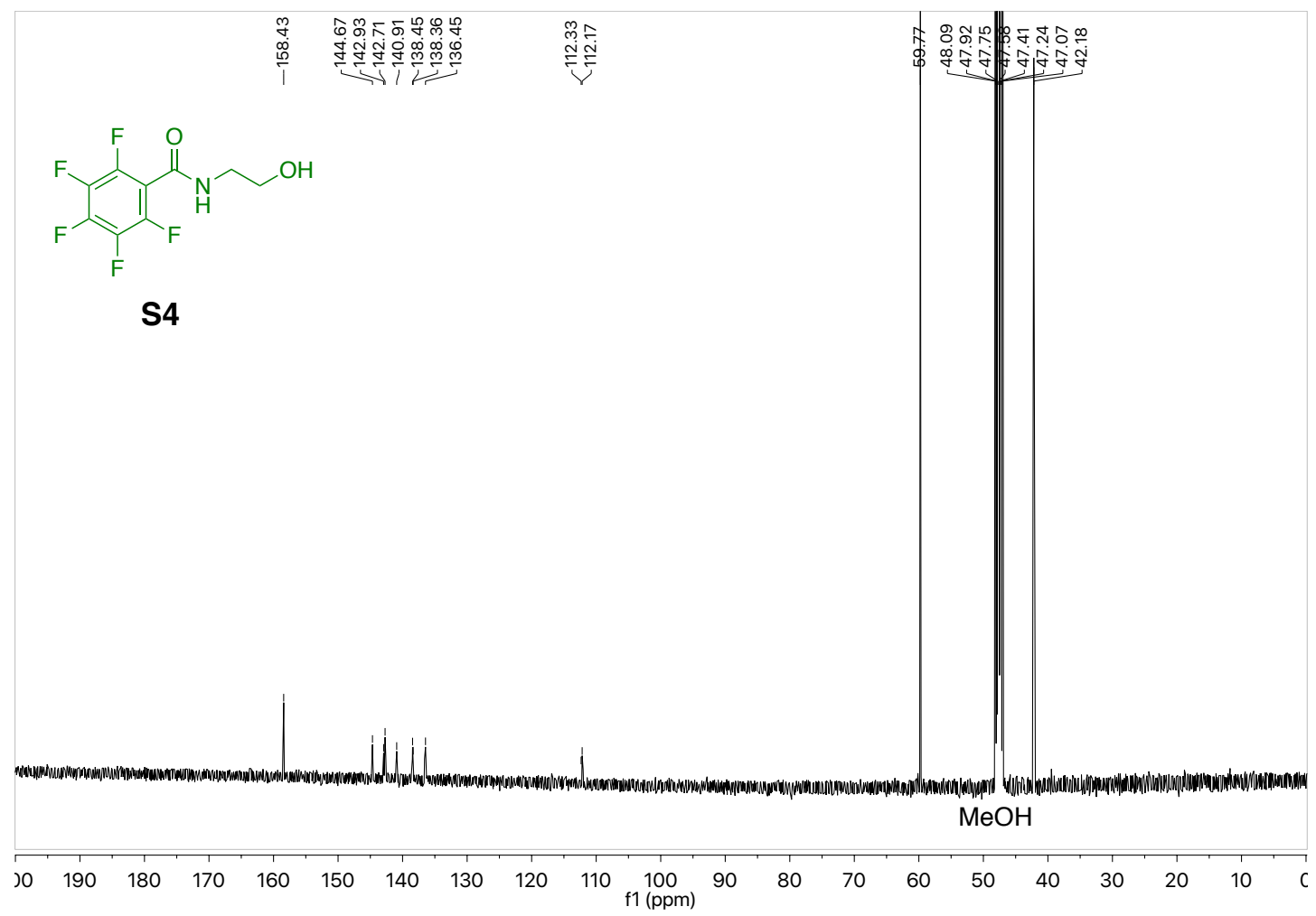

19F NMR (376 MHz, Chloroform-d)

S4
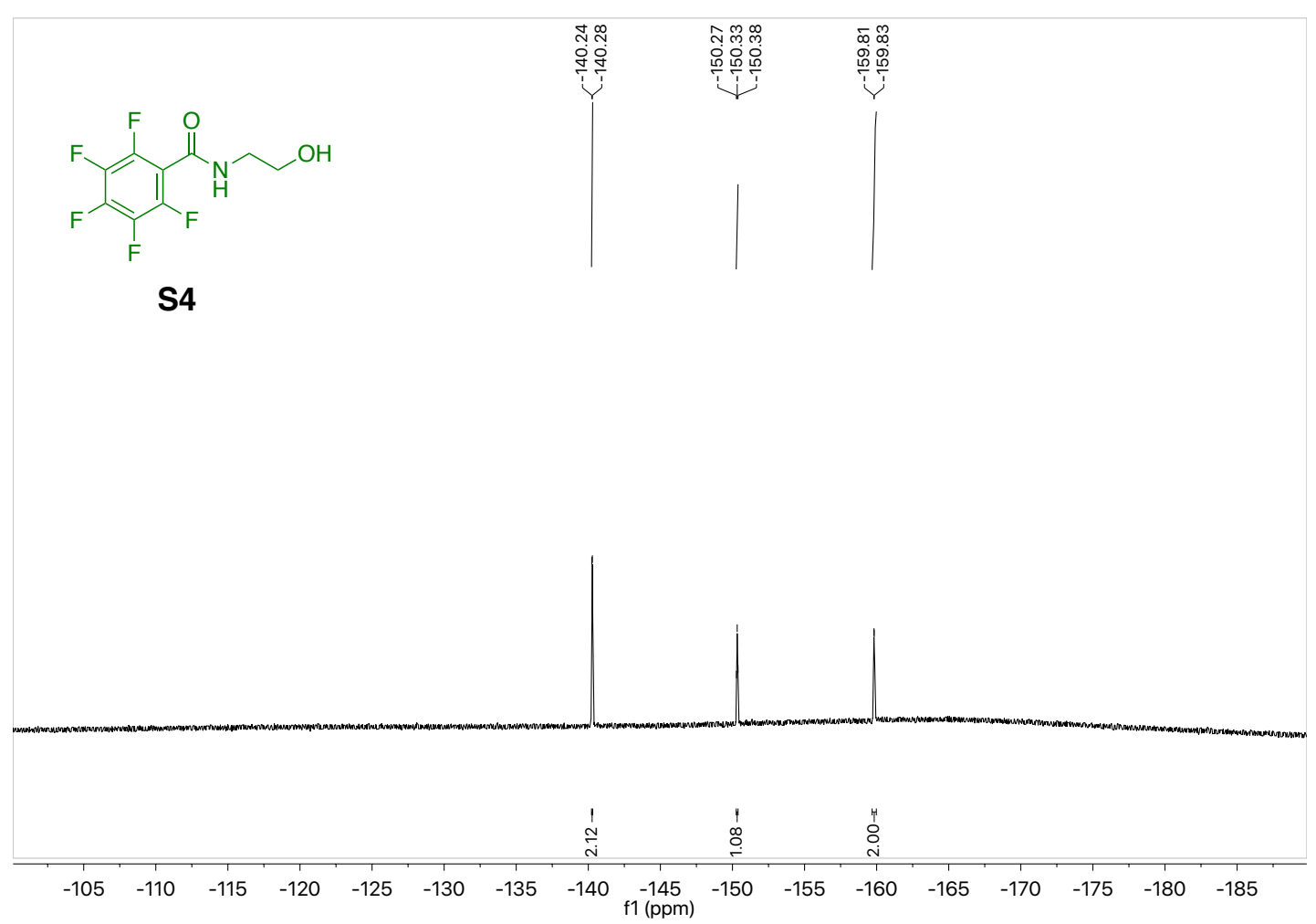

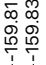

$$
\mid
$$

$\mathrm{MeOH}$ 
${ }^{1} \mathrm{H}$ NMR $\left(500 \mathrm{MHz}\right.$, Methanol- $\left.d_{4}\right)$

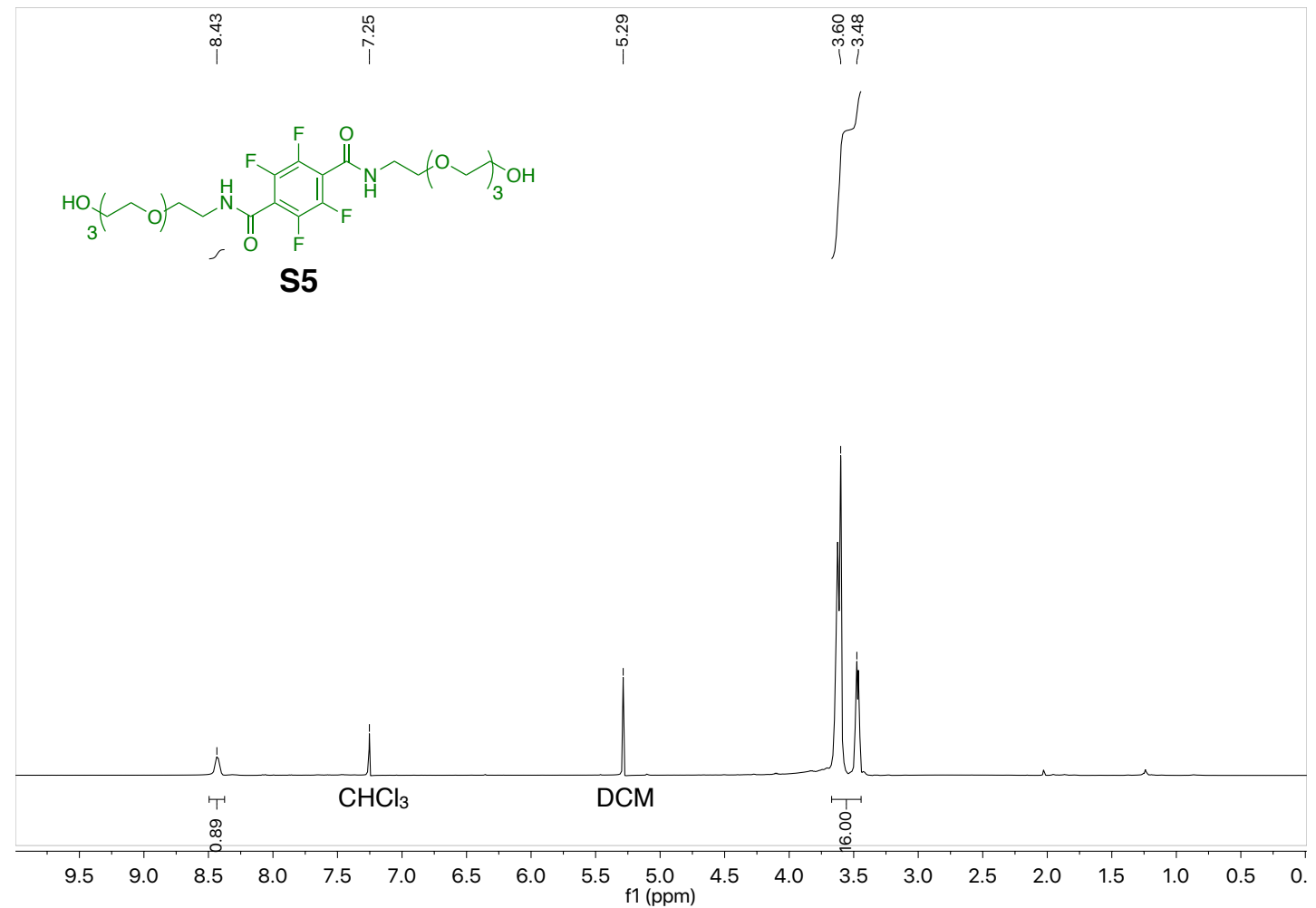

${ }^{13} \mathrm{C}$ NMR (126 MHz, Chloroform- $d$ )

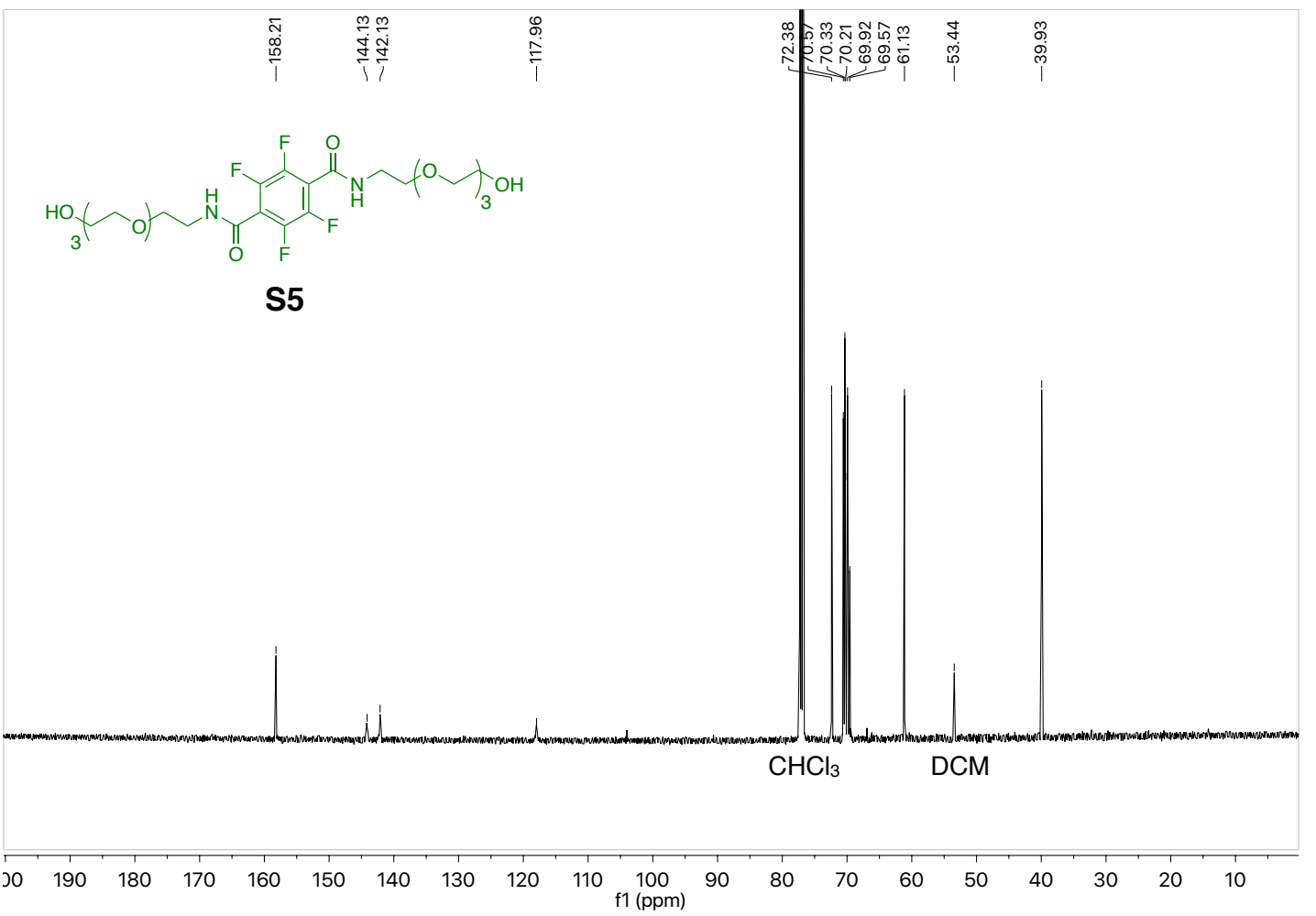


19F NMR (376 MHz, Chloroform-d)

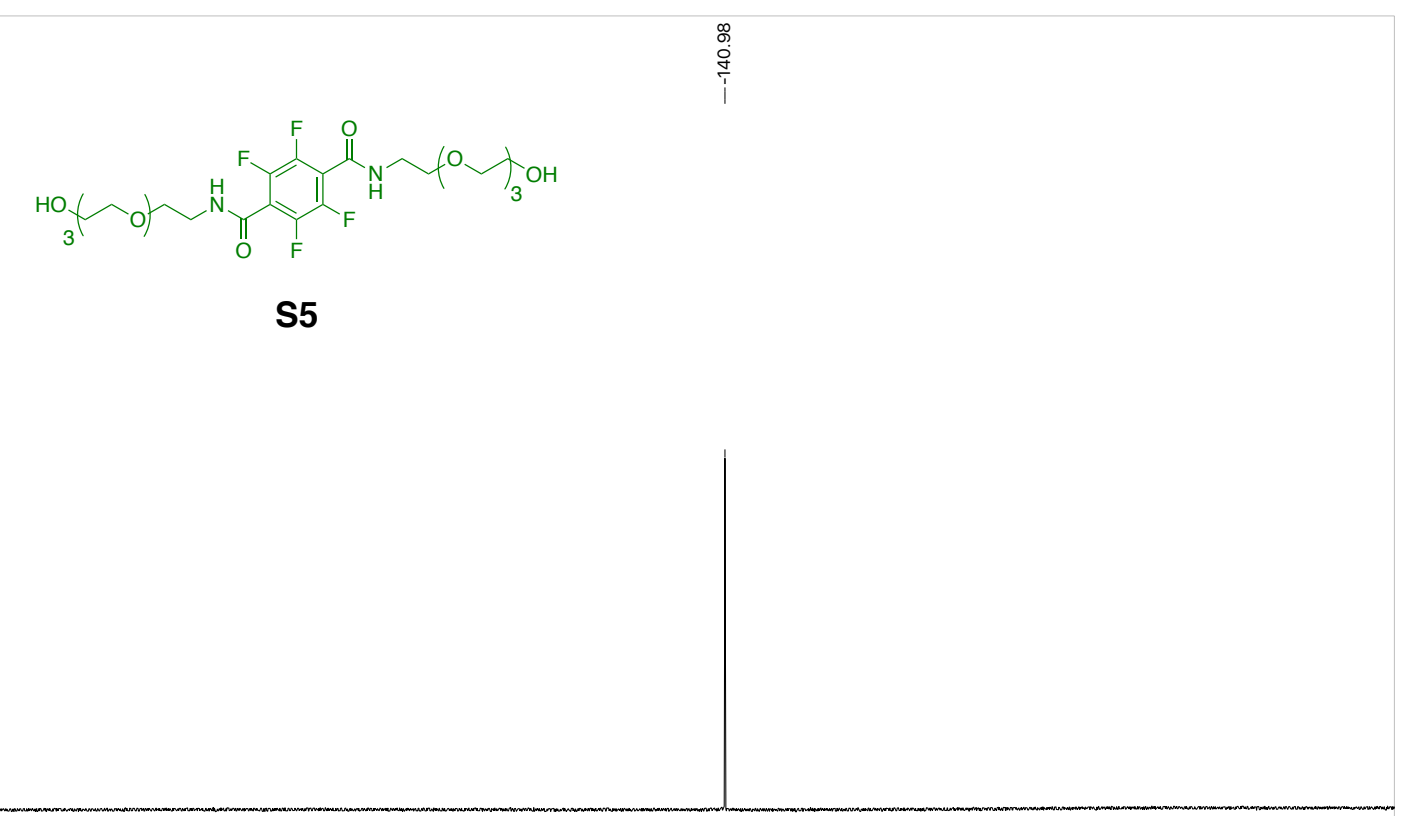

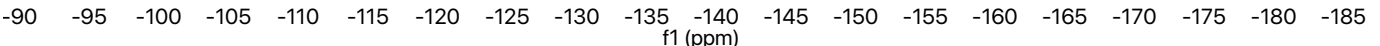

